\title{
Quantitative studies of the optical and UV spectra of Galactic early B supergiants
}

\section{Fundamental parameters $\star$}

\author{
S. C. Searle ${ }^{1}$, R. K. Prinja ${ }^{1}$, D. Massa ${ }^{2}$, and R. Ryans ${ }^{3}$ \\ 1 Department of Physics \& Astronomy, University College London, Gower Street, London WC1E 6BT, UK \\ e-mail: [scs;rkp]@star.ucl.ac.uk \\ 2 SGT, Inc., Code 665.0, Nasa Goddard Space Flight Center, Greenbelt, MD 20771, USA \\ e-mail: massa@taotaomona.gsfc.nasa.gov \\ 3 Department of Physics \& Astronomy, The Queen's University of Belfast, BT7, 1NN, Northern Ireland, UK \\ e-mail: r.ryans@qub.ac.uk
}

Received 18 January 2007 / Accepted 3 January 2008

\section{ABSTRACT}

\begin{abstract}
Aims. We undertake an optical and ultraviolet spectroscopic analysis of a sample of 20 Galactic B0-B5 supergiants of luminosity classes Ia, Ib, Iab, and II. Fundamental stellar parameters are obtained from optical diagnostics and a critical comparison of the model predictions to observed UV spectral features is made.

Methods. Fundamental parameters (e.g., $T_{\text {eff }}, \log L_{*}$, mass-loss rates and $\mathrm{CNO}$ abundances) are derived for individual stars using CMFGEN, a nLTE, line-blanketed model atmosphere code. The impact of these newly derived parameters on the Galactic B supergiant $T_{\text {eff }}$ scale, mass discrepancy, and wind-momentum luminosity relation is examined.

Results. The B supergiant temperature scale derived here shows a reduction of about 1000-3000 K compared to previous results using unblanketed codes. Mass-loss rate estimates are in good agreement with predicted theoretical values, and all of the 20 B0-B5 supergiants analysed show evidence of CNO processing. A mass discrepancy still exists between spectroscopic and evolutionary masses, with the largest discrepancy occurring at $\log \left(L / L_{\odot}\right) \sim 5.4$. The observed WLR values calculated for B0-B0.7 supergiants are higher than predicted values, whereas the reverse is true for B1-B5 supergiants. This means that the discrepancy between observed and theoretical values cannot be resolved by adopting clumped (i.e., lower) mass-loss rates as for $\mathrm{O}$ stars. The most surprising result is that, although CMFGEN succeeds in reproducing the optical stellar spectrum accurately, it fails to precisely reproduce key UV diagnostics, such as the N V and C IV P Cygni profiles. This problem arises because the models are not ionised enough and fail to reproduce the full extent of the observed absorption trough of the P Cygni profiles.

Conclusions. Newly-derived fundamental parameters for early B supergiants are in good agreement with similar work in the field. The most significant discovery, however, is the failure of CMFGEN to predict the correct ionisation fraction for some ions. Such findings add further support to revising the current standard model of massive star winds, as our understanding of these winds is incomplete without a precise knowledge of the ionisation structure and distribution of clumping in the wind.
\end{abstract}

Key words. techniques: spectroscopic - stars: mass-loss - stars: supergiants - stars: abundances - stars: atmospheres stars: fundamental parameters

\section{Introduction}

The study of luminous, massive stars is fundamental to improving our understanding of galactic evolution, since the radiatively driven winds of these stars have a tremendous impact on their host galaxies. This huge input of mechanical energy is responsible for creating $\mathrm{H}$ II regions, making a significant contribution to the integrated light of starburst galaxies and providing star formation diagnostics at both low and high redshifts. They substantially enrich the local ISM with the products of nucleosynthesis via their stellar winds and supernovae explosions and are a possible source of gamma ray bursts. It is therefore imperative to obtain accurate fundamental parameters for luminous massive stars since they contribute to many currently active areas of astrophysical research.

\footnotetext{
* Appendix A is only available in electronic form at http://www. aanda.org
}

However there are still some uncertainties regarding the post-main sequence evolution of massive stars since their evolution is controlled by variable mass loss from the star as well as rotation, binarity and convective processes, the latter leading to surface enrichment as the products of nuclear burning are brought to the surface. Until recently, stellar evolution models failed to predict the correct amount of CNO processing in massive stars. However, new evolutionary tracks that account for the effects of rotation (Maeder \& Meynet 2001) show better agreement between predicted and observed amounts of CNO enrichment in massive stars. A far greater problem in stellar astrophysics is the determination of accurate observed mass loss rates. Recent research (e.g., Fullerton et al. 2006; Prinja et al. 2005; Massa et al. 2003; Puls et al. 2006; Repolust et al. 2004), has shown that current $\mathrm{OB}$ star mass loss rates might be overestimated by at least a factor of 10 . Such large uncertainties in the mass loss rates of massive stars suggest that our understanding of their winds is incomplete. It is now widely accepted 
that the winds of both $\mathrm{O}$ and $\mathrm{B}$ stars are highly structured and clumped and therefore our assumptions that they are smooth and homogeneous are invalid. Evidence to support this claim has come from hydrodynamical, time-dependent simulations of stellar winds (e.g. Owocki et al. 1988; Runacres \& Owocki 2002), the latter of which proposed the idea that instabilites in the linedriving of the wind can produce small-scale, stochastic structure in the wind. Further evidence for the inhomogeneity of stellar winds comes in the form of various observational studies (e.g., Puls et al. 2006; Bouret et al. 2005; Massa et al. 2003; Prinja et al. 2002; Bianchi 2002). Time-series analyses of both Balmer and metal spectral lines (e.g., Prinja et al. 2004) in OB stars highlight clear, periodic patterns of variability that correspond to the evolution of structure in the wind. Puls et al. (2006) demonstrated that the discrepancy between values of $\dot{M}_{\mathrm{H} \alpha}$ and $\dot{M}_{\text {radio }}$ implies the presence of different amounts of clumping at the base of and further out in the wind. Massa et al. (2003) showed that for a sample of $\mathrm{O}$ stars in the LMC, the empirical ionisation fractions derived were several orders of magnitude lower than expected, indicating a lack of dominant ions in the wind. Similar results were found by Prinja et al. (2005) for early B supergiants. More recently, Fullerton et al. (2006) demonstrated that the ionisation fraction of $\mathrm{PV}$, which is dominant over a given range in $T_{\text {eff }}$ in the $\mathrm{O}$ star spectral range, never approaches a value of unity. They subsequently showed that a reduction in mass loss rate of at least a factor of 100 is required to resolve the situation. We intend to re-address the issue of the ionisation structure of early B supergiants, following on from Prinja et al. (2005), in a forthcoming paper (Searle et al. 2007b, in preparation, hereafter Paper II). Such drastic reductions in OB star mass loss rates would have severe consequences for the post-mainsequence evolution of these stars; in particular it would affect the numbers of Wolf-Rayet stars produced and the ratio of neutron stars to black holes produced in the final stages of massive star evolution.

Early type B supergiants are particularly important since they are the most numerous massive luminous stars and are ideal candidates for extra-galactic distance indicators, essential for calibrating the Wind-Momentum-Luminosity Relation (WLR) (e.g., Kudritzki et al. 1999). Research into this WLR calibration has highlighted a spectral type dependence for Galactic OBA type stars (e.g., Kudritzki et al. 1999; Repolust et al. 2004; Markova et al. 2004), whilst others have explored the effect of metallicity on the WLR by studying OB stars in the metal-poor environment of the Magellanic Clouds (e.g., Kudritzki \& Puls 2000; Trundle et al. 2004; Evans et al. 2004b). Accurately derived mass loss rates are essential in calibrating the WLR, yet discrepancies still exist between observed mass loss rates obtained from different wavelength regions (i.e. optical, UV or IR). Furthermore acknowledged discrepancies of up to $30 \%$ have been found between observational and theoretically predicted mass loss rates (Vink et al. 2000). Vink has remarked that these discrepancies for the mass loss rates of B stars can be attributed to systematic errors in the methods employed to derive the observed values. Good agreement was found between observed and predicted mass loss rates for O stars in Vink et al. (2000). Additionally, Puls et al. (2006) recently derived values of both $\dot{M}_{\mathrm{H} \alpha}$ and $\dot{M}_{\text {radio }}$, highlighting a discrepancy of roughly a factor of two between both values.

The layout of this paper is as follows. Section 2 introduces the sample of 20 Galactic B0-B5 supergiants upon which this analysis is based. Section 3 describes the methodology used in deriving fundamental parameters for this sample. The results are presented in Sect. 4 and a critical examination of the
CMFGEN model fit to the UV spectra of the $20 \mathrm{~B}$ supergiants is made in Sect. 5. Finally the conclusions are given in Sect. 6.

\section{Observations}

Optical and UV spectra have been collected for a sample of 20 Galactic B supergiants, covering the spectral range of B0-B5 and including Ia, Ib, Iab and II luminosity classes as well as a hypergiant. Stars were only included in the sample if both optical and $I U E$ data were available for them. Where possible, stars were selected such that there would be 2 different luminosity classes at each spectral sub-type. The details of observational data for each star are given in Table 1. Fourteen of the chosen B supergiants belong to OB associations (Humphreys 1978), so for these stars, the absolute magnitude given in Table 1 is based on the distance to the relevant association; for the remaining six stars $M_{V}$ and therefore the distance modulus is derived from photometry.

For fifteen of the twenty B supergiants in our sample, the optical spectra were taken from an existing data set (see Lennon et al. 1992, for further details). The blue spectra were observed using the 1-m Jacobus Kapteyn Telescope (JKT) at the Observatorio del Roque de los Muchacos, La Palma in October 1990 with the Richardson-Brealey Spectrograph and a R1200B grating. They have a wavelength coverage of 3950-4750 $\AA$, a spectral resolution of $0.8 \AA$ and a signalto-noise ratio $\sim 150$. The red spectra were obtained with the 2.5-m Isaac Newton Telescope (INT) using the Intermediate Dispersion Spectrograph (IDS) and cover a wavelength range of 6260-6870 $\AA$ (Lennon et al. 1992). The spectral resolution is $0.7 \AA$ and the signal-to-noise ratio is $>100$. Spectra for HD 192660, HD 185859, HD 190066 and HD 191243 were taken at the INT in July 2003, again using the IDS. The R400B grating was used for the blue spectra, giving a central wavelength $\lambda_{\mathrm{c}}$ of $4300 \AA$, whereas the R600R grating was used for the red spectra, giving $\lambda_{\mathrm{c}} \sim 6550 \AA$. The signal-to-noise ratio was $>100$ and the spectral resolution was $0.7 \AA$. Finally high resolution time-averaged blue and red spectra of HD 64760 were provided by RKP (see Kaufer \& Stahl 2002, for more details). These spectra were taken in 1996 on the HEROS fiber-linked echelle spectrograph, which was mounted on the ESO $50-\mathrm{cm}$ telescope at the La Silla, Chile. The blue spectra have a range

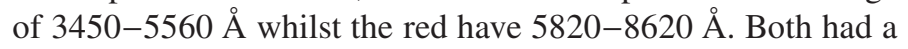
resolving power of 20000 . The signal-to-noise ratio varied with lambda but for a red spectrum with a 40-min exposure it was typically $>150$.

\subsection{Sample details}

As previously mentioned, our sample covers a range of B0-B5 supergiants with luminosity classes varying from Ia down to II in a couple of cases. Spectral type classifications have been taken from Lennon et al. (1992), which includes some recent revisions. HD 204172 has been changed from B0 Ib to B0.2 Ia due to the strength of its Si IV lines and narrowness of its $\mathrm{H}$ lines. Comparing its UV resonance lines to those of the B0 Ib star HD 164402 supports the change to a more luminous spectral type (Prinja et al. 2002). Also both HD 164353 and HD 191243 have been reclassified from B5 Ia to B5 II stars. It is also worth noting that de Zeeuw et al. (1999) has questioned the membership of the stars HD 53138 and HD 58350 to Collinder 121 on account of insignificant proper motion and small parallax respectively. Seven of the twenty stars in our sample have been examined for $\mathrm{H} \alpha$ variability by Morel et al. (2004), 
Table 1. Observational data for the sample of 20 Galactic B Supergiants. Spectral types and $V$ magnitudes are taken from Lennon et al. (1992) for all stars except HD 192660, HD 185859, HD 190066 and HD 64760. The references for the spectral types of these 4 stars are as follows: HD 192660 from Walborn (1971); HD 185859 from Lesh (1968); HD 190066 from Hiltner (1956) and HD 64760 from Hoffleit \& Jaschek (1982) (from which the $V$ magnitude of HD 64760 was also taken). $V$ magnitudes for the remaining 3 stars were obtained from Fernie (1983). Values of $(B-V)_{0}$ taken from Fitzgerald (1970). Absolute visual magnitudes, distance moduli and cluster associations have been taken from: 1. Brown et al. (1994), 2. Garmany \& Stencel (1992) or 3. Humphreys (1978). For stars not associated with a cluster, an absolute visual magnitude scale based on spectral type (Egret 1978) was used. L1992 refers to archive data obtained from Lennon et al. (1992), INT2003 denotes data taken on the $2.5 \mathrm{~m}$ INT and RKP marks data supplied by Prinja.

\begin{tabular}{rlllllllll}
\hline \hline HD No. & Alias & Sp. type & $V$ & $B-V$ & $M_{V}$ & Association & m-M & Optical & $I U E$ \\
\hline 37128 & $\epsilon$ Ori & B0 Ia & 1.70 & -0.19 & -6.95 & Ori OB1b & $7.8^{1}$ & L1992 & SWP30272 \\
192660 & - & B0 Ib & 7.38 & 0.67 & -7.0 & Cyg 0B8 & $11.8^{3}$ & INT2003 & SWP44625 \\
204172 & 69 Cyg & B0.2 Ia & 5.94 & -0.08 & -6.2 & Cyg OB4 & 6.2 & L1992 & SWP48900 \\
38771 & $\kappa$ Ori & B0.5 Ia & 2.04 & -0.18 & -6.51 & Ori OB1c & $8.0^{1}$ & L1992 & SWP30267 \\
185859 & - & B0.5 Ia & 6.48 & +0.44 & -6.6 & - & 13.08 & INT2003 & SWP47509 \\
213087 & 26 Cep & B0.5 Ib & 5.46 & +0.37 & -6.2 & Cep OB1 & 12.7 & L1992 & SWP02735 \\
64760 & - & B0.5 Ib & 4.24 & -0.15 & -6.2 & - & 10.44 & RKP & SWP53781 \\
2905 & $\kappa$ Cas & BC0.7Ia & 4.16 & +0.14 & -7.09 & Cas OB14 & $10.2^{3}$ & L1992 & SWP54038 \\
13854 & V551 Per & B1 Iab(e) & 6.47 & +0.28 & -6.73 & Per OB1 & $11.8^{2}$ & L1992 & SWP02737 \\
190066 & - & B1 Iab(e) & 6.53 & +0.18 & -6.1 & - & 12.63 & INT2003 & SWP18310 \\
190603 & V1768 Cyg & B1.5 Ia+ & 5.64 & +0.54 & -7.0 & - & 12.56 & L1992 & SWP4325 \\
193183 & - & B1.5 Ib & 7.01 & +0.44 & -6.24 & Cyg OB1 & $11.3^{3}$ & L1992 & SWP52716 \\
14818 & V554 Per & B2 Ia & 6.25 & +0.30 & -6.93 & Per OB1 & $11.8^{3}$ & L1992 & SWP18658 \\
206165 & V337 Cep & B2 Ib & 4.74 & +.30 & -6.44 & Cep OB2 & 13.2 & L1992 & SWP06336 \\
198478 & 55 Cyg & B2.5 Ia & 4.84 & +0.40 & -6.43 & Cyg OB7 & $9.6^{2}$ & L1992 & SWP38688 \\
42087 & 3 Gem & B2.5 Ib & 5.75 & +0.22 & -6.26 & Gem OB1 & $10.9^{3}$ & L1992 & SWP08645 \\
53138 & 24 CMa & B3 Ia & 3.01 & -0.11 & -7.1 & - & 10.11 & L1992 & SWP30271 \\
58350 & $\eta$ CMa & B5 Ia & 2.41 & -0.07 & -7.0 & - & 9.41 & L1992 & SWP30198 \\
164353 & 67 Oph & B5 II(Ib) & 3.97 & +0.02 & -4.2 & Coll 359 & 6.5 & L1992 & SWP08560 \\
191243 & - & B5 II(Ib) & 6.09 & +0.16 & -6.5 & Cyg OB3 & $11.8^{3}$ & INT2003 & SWP07737 \\
\hline
\end{tabular}

who obtained both photometric and spectroscopic data on these objects in order to ascertain the amount of variability present in their light-curves and $\mathrm{H} \alpha$ profiles. Morel et al. (2004) quantify the amount of spectral and photometric variability observed as well as determining periods where cyclic behaviour is observed. The sample includes a rapid rotator, HD 64760 (discussed in detail in the following section) and a hypergiant, the B1.5 Ia+ star HD 190603. Furthermore, there are several objects of interest in the sample for which a significant amount of research has already been undertaken and merit further discussion.

- $\epsilon$ Ori: There are several intriguing aspects of this star that are worth mentioning. Firstly it has been noted as moderately nitrogen deficient by Walborn (1976). Secondly it is known to undergo significant variations in $\mathrm{H} \alpha$, with Morel et al. (2004) reporting variations of $81.9 \%$. Further studies by Prinja et al. (2004) have revealed variability in not only $\mathrm{H} \alpha$ but also $\mathrm{H} \beta$, He absorption and metal lines with a 1.9 day period. A modulating S-wave pattern has been discerned in the weaker lines, which cannot be fully explained by current non-radial pulsation models (Townsend 1997). These results highlight a direct connection between photospheric activity and perturbations in the stellar wind. Finally this star is the only normal early B supergiant to have a measured thermal radio flux (Blomme et al. 2002), from which a radio mass loss rate of $\log \dot{M}=-5.72$ was derived.

- $\kappa$ Ori: Like $\epsilon$ Ori, $\kappa$ Ori also exhibits spectral variability in $\mathrm{H} \alpha$. Its $\mathrm{H} \alpha$ profile was studied in detail by Rusconi et al. (1980) who described it as a double-peaked absorption profile with a central emission core and broad profile wings, with variations on long (of the order of days) and short (of the order of minutes) time scales. More recently Morel et al. (2004) reported changes in the profile amplitude and morphology of $32.6 \%$. Walborn (1976) noted it as an example of a morphologically normal B supergiant in terms of the relative strengths of its $\mathrm{CNO}$ spectral lines.

- HD 192660: Bidelman (1988) noted that this star showed a "faint $\mathrm{H} \alpha$ emission with a slight P Cygni absorption", based on observations taken at the Lick observatory in 1957. Our spectrum of this star shows a very similar $\mathrm{H} \alpha$ profile that is also only weakly in emission. Walborn (1976) and Schild (1985) both noted that HD 192660 displays evidence for nitrogen deficiency.

- HD 64760: This star is classified as a rapid rotator, having a $v \sin i$ of $265 \mathrm{~km} \mathrm{~s}^{-1}$. Many interesting studies have been carried out regarding the periodic and sinusoidal modulations of its optical and UV lines (Massa et al. 1995; Fullerton et al. 1997; Kaufer \& Stahl 2002), which have turned HD 64760 into a key object for improving our understanding of the spatial structure and variations of hot star winds. More recently, Kaufer et al. (2006) found, for the first time, direct observational evidence for a connection between multi-periodic nonradial pulsations (NRPs) in the photosphere and spatially structured winds. More specifically, they can use the interference of multiple photospheric pulsation modes on hourly timescales with wind modulation periods on time scales of several days. A beat period of 6.8 days seen in the photosphere and base of the wind does not match with the derived periods of 1.2 and 2.4 days for wind variability, being closer to the longer 5-11 day repetitive timescales observed for discrete absorption components (DACs) in the IUE data sets. Evidently the precise nature of the wind-photosphere connection in this star is a complex one. Using hydrodynamical simulations, Cranmer \& Owocki (1996) succeeded in confirming the existence of co-rotating interaction regions (CIRs). These are spiral structures in the wind that are produced through the collisions of fast and slow streams rooted in the stellar surface. 
- $\kappa$ Cas: Walborn (1972) classified this star as carbon rich, giving it a spectral type of $\mathrm{BC} 0.7 \mathrm{Ia}$, when comparing its optical spectrum with that of HD 216411, a B0.7 Ia star. $\mathrm{He}$ found that the nitrogen lines in $\kappa$ Cas were barely detectable, whereas the O II-C III blends were very prominent. Since Walborn also recognised that HD 216411 possesses a well developed nitrogen spectrum, he described $\kappa$ Cas as carbon-rich, rather than nitrogen-weak, with respect to a morphologically-normal B supergiant. Walborn had previously suggested that all $\mathrm{OB}$ stars begin their post-mainsequence evolution with an enhancement of carbon (Walborn 1971), which then becomes depleted as the star evolves and produces nitrogen as a by-product of the $\mathrm{CNO}$ bi-cycle. Therefore the implication of classifying $\kappa$ Cas as a BC 0.7 Ia star is that it is less evolved than other stars in the sample. Please consult Sect. 4.5 for a discussion of the carbon rich status of this star based on the results presented in that section.

- HD 13854: McErLean et al. (1999) describe this star as "highly processed" i.e. displaying a large amount of CNO processing in its spectrum. Its $\mathrm{H} \alpha$ profile is seen mostly in emission, assuming a P Cygni shape. Morel et al. (2004) found that not only does the $\mathrm{H} \alpha$ profile of this star vary by $47.3 \%$, but that these variations have a period of 1.047 days \pm 0.01 . Hipparcos light curves also show evidence for periodic behaviour.

- HD 14818: possesses an $\mathrm{H} \alpha$ profile with a P Cygni profile. Morel et al. (2004) report variations of $34.8 \%$ in the $\mathrm{H} \alpha$ profile and, like HD 13854, they find that this star also shows periodic behaviour in its Hipparcos light curve.

- HD 42087: also has its $\mathrm{H} \alpha$ profile in emission but more importantly Morel et al. (2004) reported significant $\mathrm{H} \alpha$ variability of $91.2 \%$ (greater than the percentage variability that they found for $\epsilon$ Ori), for which they find strong evidence of cyclic behaviour on a periodicity of 25 days $\pm 1-4$ days. Moreover, the $\mathrm{H} \alpha$ variability correlates with variability in the He I $6678 \AA$ line, such that as $\mathrm{H} \alpha$ emission increases, He I 6678 becomes weaker. Morel et al. (2004) also find periodic variability in its Hipparcos light curve.

- HD 53138: Walborn (1976) notes that this star shows a morphologically normal CNO spectrum, despite other authors associating this star with $\mathrm{OBN} / \mathrm{OBC}$ groups. It undergoes $66.4 \% \mathrm{H} \alpha$ variability (Morel et al. 2004).

\section{Derivation of fundamental parameters}

Fundamental parameters were derived for this sample of stars using the nLTE stellar atmosphere codes TLUSTY (Hubeny \& Lanz 1995; Lanz 2003) and CMFGEN (Hillier \& Miller 1998). The application of TLUSTY, a plane-parallel photospheric code that does not account for the presence of a stellar wind, to modelling supergiants is valid as long as it is used solely to model purely photospheric lines. An existing grid of B star TLUSTY models (Dufton et al. 2005) was used as a base for this work and the grid (originally incremented in steps of $\sim 2000 \mathrm{~K}$ in $T_{\text {eff }}$ and 0.13 in $\log g$ ) was refined further by RR in the range $15000 \mathrm{~K} \leq T_{\text {eff }} \leq 23000 \mathrm{~K}$ to cover the same parameter space as the CMFGEN grid. The TLUSTY models provide a hydrostatic structure that can be input into CMFGEN, since the latter code does not solve for the momentum equation and therefore requires a density/velocity structure (see e.g., Hillier et al. 2003; Bouret et al. 2003; Martins et al. 2005). The TLUSTY input provides the subsonic velocity structure and the supersonic velocity structure in the CMFGEN model is described by a $\beta$-type law.

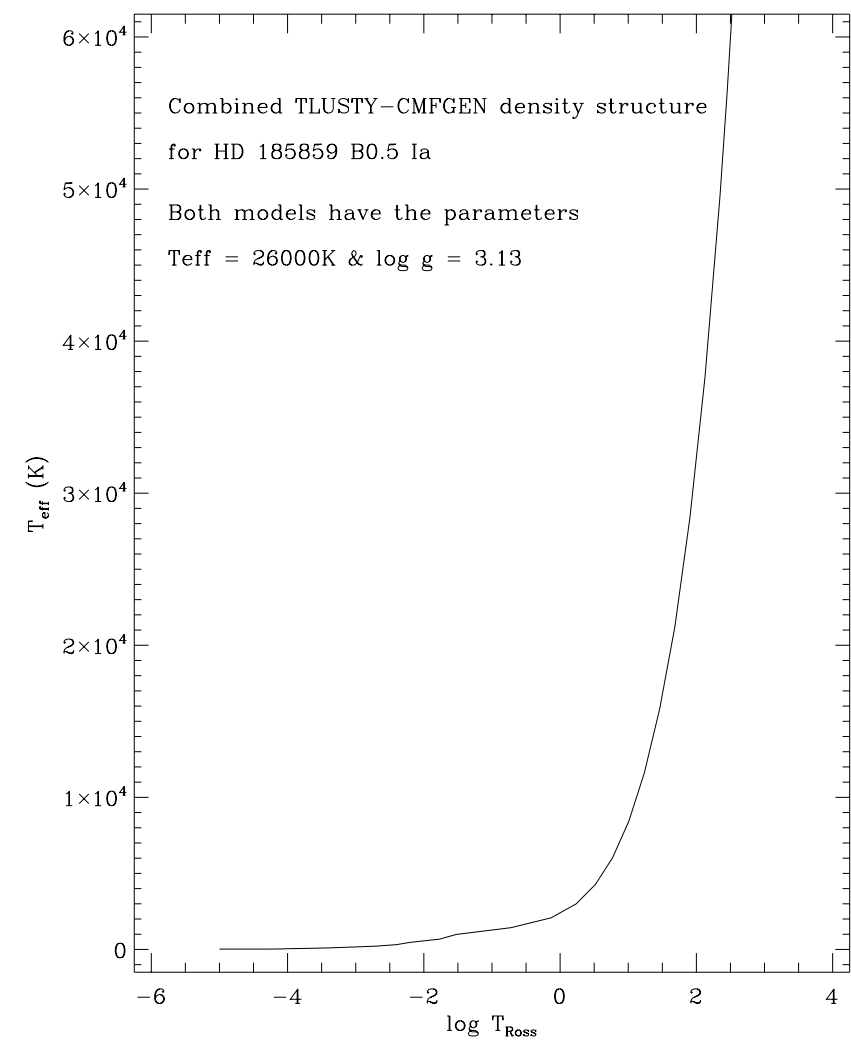

Fig. 1. Temperature structure against Rosseland optical mean depth for the hydrostatic density structure produced from combining the subsonic TLUSTY velocity structure with the supersonic CMFGEN velocity structure, such that the velocity and velocity gradient are constant. The above example is for the B0.5 Ia star HD 185859 ( $T_{\text {eff }}=26000 \mathrm{~K}$, $\log g=3.13)$.

The two structures are joined to a hydrostatic density structure at depth, such that the velocity and velocity gradient are consistent. The resulting structure is shown in Fig. 1, which shows the change in Rosseland mean opacity, $\tau_{\text {Ross }}$, with temperature and ensures that the model is calculating deep enough into the photosphere to sample the regions where the appropriate photospheric lines form (around $-2 \leq \log \tau_{\text {Ross }} \leq 0$ ). This structure is then input into the CMFGEN model, adopting a $\beta$-type velocity law of the form:

$v(r)=\frac{v_{0}+\left(v_{\infty}-v_{0}\right)\left(1-R_{*} / r\right)^{\beta}}{1+\frac{v_{0}}{v_{\text {core }}} \mathrm{e}^{\frac{R_{*}-r}{h_{\text {eff }}}}}$

where $v_{0}$ is the photospheric velocity, $v_{\text {core }}$ is the core velocity, $v_{\infty}$ is the terminal velocity, $h_{\text {eff }}$ is the scale height expressed in terms of $R_{*}$ and $\beta$ is the acceleration parameter. The value of $\beta$ is normally determined from fitting the $\mathrm{H} \alpha$ profile, as discussed in Sect. 3.2, assuming typical values of $1.0 \leq \beta \leq 1.5$ for B supergiant. Additionally, values of $v_{\text {core }}=0.002 \mathrm{~km} \mathrm{~s}^{-1}$ and $v_{0}=$ $0.1 \mathrm{~km} \mathrm{~s}^{-1}$ are adopted for B supergiants as suggested by Hillier (priv. comm.). All parameters except $\log g$ were derived using CMFGEN and the precise details of the methods employed will be discussed next. The general method employed was to produce a grid of CMFGEN models of varying temperatures and luminosities (all other parameters were kept constant), incremented in steps of $1000 \mathrm{~K}$ in $T_{\text {eff }}$ and 5 in $\log \left(L / L_{\odot}\right)$, and compare these synthetic spectra to observed spectra in order to constrain these two parameters (Sect. 3.1). Once satisfactory values had been derived for the temperature and luminosity of a star, the mass 
Table 2. CMFGEN model atomic data, showing the number of full levels and superlevels treated as well as the number of bound-bound transitions considered for each ion included in a CMFGEN model.

\begin{tabular}{lccc}
\hline \hline Ion & Full levels & Superlevels & b-b transitions \\
\hline H I & 30 & 20 & 435 \\
He I & 59 & 41 & 590 \\
He II & 30 & 20 & 435 \\
C II & 53 & 30 & 323 \\
C III & 54 & 29 & 268 \\
C IV* & 18 & 13 & 76 \\
N I & 22 & 10 & 59 \\
N II & 41 & 21 & 144 \\
N III* & 70 & 34 & 430 \\
O I & 75 & 18 & 450 \\
O II & 63 & 22 & 444 \\
O III* & 45 & 25 & 182 \\
Mg II & 45 & 18 & 362 \\
Al II & 44 & 26 & 171 \\
Al III & 65 & 21 & 1452 \\
Si II & 62 & 23 & 365 \\
Si III & 45 & 25 & 172 \\
Si IV & 12 & 8 & 26 \\
S II & 87 & 27 & 786 \\
S III & 41 & 21 & 177 \\
S IV* & 92 & 37 & 708 \\
Ca II & 12 & 7 & 28 \\
Fe II & 510 & 100 & 7501 \\
Fe III & 607 & 65 & 5482 \\
Fe IV & 272 & 48 & 3113 \\
Fe V* & 182 & 46 & 1781 \\
\hline
\end{tabular}

loss rate, $\beta$ velocity law, turbulent velocity $\left(v_{\text {turb }}\right)($ Sect. 3.2$)$ and CNO abundances (Sect. 4.5) could be constrained. These parameters are all sensitive to changes in temperature and luminosity so can only be derived once those values have been fixed. Consistency checks are made after each parameter is altered to ensure that the resulting model output has not worsened the fit to the diagnostic lines and overall spectrum. Given recent improvements in the treatment of metal lines in nLTE stellar atmosphere codes such as CMFGEN or FASTWIND, it is now possible to obtain accurate abundances by fitting (by eye) appropriate diagnostic lines for each element. All models were calculated to include the following elements; $\mathrm{H}, \mathrm{He}, \mathrm{C}, \mathrm{N}, \mathrm{O}, \mathrm{Al}, \mathrm{Mg}, \mathrm{Si}, \mathrm{S}$, $\mathrm{Ca} \& \mathrm{Fe}$, assuming solar abundances for silicon, magnesium, aluminium, phosphorous, sulphur, calcium and iron and adopting a relative number fraction of 5:1 for $\mathrm{H}: \mathrm{He}$ (as used by Hillier et al. 2003). CMFGEN adopts the superlevel approximation (see Anderson 1989 for more details), where a superlevel can consist of several or even many energy levels grouped together, such that all real levels $j$ that form superlevel $J$ have the same nLTE departure coefficient (i.e., each component $j$ is in Boltzmann equilibrium with respect to the other components). Details of the model atoms, including their full level and superlevel groupings, are given in Table 2.

\subsection{Derivation of $T_{\text {eff }}, \log \left(L / L_{\odot}\right), \log g$ and $C N O$ abundances}

In B stars, the silicon lines are used as the primary temperature diagnostics, having the advantage that the abundance is well known as silicon is unaffected by nuclear processing. For B0-B2 supergiants the Si IV $4089 \AA$ and Si III 4552, 4568 and $4575 \AA$ lines provide the main temperature diagnostics. Si IV $4089 \AA$ A decreases in strength as the temperature decreases until it is barely detectable at a spectral type of B2.5, which corresponds to $T_{\text {eff }} \sim 18000 \mathrm{~K}$. At this point the Si II 4128-30 doublet is present and replaces Si IV $4089 \AA$ as the main temperature diagnostic for B2.5-B9 stars, along with Si III 4552, 4568 and $4575 \AA$. The He I lines at $4144 \AA$, $4387 \AA, 4471 \AA$ and $4713 \AA$ and Mg II line at $4481 \AA$ can also be used as secondary criteria for both temperature and luminosity, since they are sensitive to changes in both parameters. The principal luminosity criteria used in spectral classification of B stars is the ratio of Si IV $4089 \AA$ to He I $4026 \AA, 4121 \AA$ and/or $4144 \AA$ for B0-B1 supergiants, whereas for stars later than B1 the ratio of Si III 4552,4568 and $4575 \AA$ to He I $4387 \AA$ is used. The procedure adopted for deriving values of $T_{\text {eff }}, \log \left(L / L_{\odot}\right), \log g$ and $\mathrm{CNO}$ abundances is the same method adopted by Hillier et al. (2003); Crowther et al. (2006); Bouret et al. (2003); Martins et al. (2005) and is as follows:

1. an optical stellar spectrum of a chosen star is compared to a grid of CMFGEN models which differ in values of $T_{\text {eff }}$ and luminosity (all other parameters are kept constant);

2. a value of $T_{\text {eff }}$ is selected for the star by finding the model that provides the best fit to the temperature sensitive silicon lines. The diagnostic lines are Si IV 4089 and Si III 4552, 4568 and 4575 for B0-B2 supergiants and Si II 4128-30 and Si III 4552, 4568 and 4575 for B2.5-B5 supergiants. This temperature would then be confirmed by checking that the model also succeeded in fitting the helium lines listed earlier and $\mathrm{Mg}$ II (but these lines were not used to derive the initial value of $T_{\text {eff }}$ ) to ensure it provided a reasonable match to all temperature-sensitive lines;

3. once a value of $T_{\text {eff }}$ has been chosen, an inital estimate of $\log \left(L / L_{\odot}\right)$ is made by selecting the model from the grid (at the chosen value of $T_{\text {eff }}$ ) whose value of $M_{V}$ (output from the model) best matches the observed value of $M_{V}$ for the star in question. The luminosity is then constrained further by taking observed values of $M_{V}$, the absolute visual magnitude and $V$, together with the estimate of $A(V)$, were used to obtain an initial estimate of the distance modulus. Optical photometry and ultraviolet spectroscopy were then de-reddened with respect to the model spectral energy distribution to obtain revised estimates of $E(B-V)$ and $M_{V}$. This $M_{V}$ derived from the model was then compared to an observed $M_{V}$, if the values matched then the model luminosity was correct. If not, the value of $M_{V}$ was translated into a bolometric correction to obtain an estimate of the corrected luminosity for the model, which was then rerun with this value for the luminosity. This iterative process was continued until the observed and model values of $M_{V}$ were in reasonable agreement and then the resulting model was checked against the $T_{\text {eff }}$ diagnostic lines ( $\mathrm{Si}$ II, Si III, Si IV) and derived value of $T_{\text {eff }}$ was adjusted if necessary;

4. estimates of $\log g$ were then made from TLUSTY fits to the $\mathrm{H} \gamma$ lines of the observed spectra. $\mathrm{H} \gamma$ is normally chosen as the $\log g$ diagnostic since $\mathrm{H} \alpha$ and $\mathrm{H} \beta$ suffer from too much wind fill emission; $\mathrm{H} \delta$ was used as a secondary diagnostic to check for consistency with values of $\log g$ derived from $\mathrm{H} \gamma$. Since $\mathrm{H} \gamma$ is affected by an O II blend around $4350 \AA$ and $\mathrm{H} \delta$ has a blend with N III 4097, neither line is ideal but both implied the same $\log g$ values so we can have confidence that the derived $\log g$ values are not affected by these blends. The adopted $\log g$ value was then incorporated into the CMFGEN model and again the derived $T_{\text {eff }}$ and $\log \left(L / L_{\odot}\right)$ values were revised if necessary; 
5. next $\mathrm{CNO}$ abundances were derived by varying the abundance of each element until the appropriate diagnostic lines were fitted by the model. For nitrogen, we use the N III $4097 \AA$ line for B0-B2 stars (which is blended with $\mathrm{H} \delta$ ) and the N II $3995 \AA, \mathrm{N}_{\text {II }} 4447 \AA$ and $4630 \AA$ lines (for all B stars) as primary diagnostics. The main diagnostics for carbon and oxygen are the $4267 \AA$ and $4367 \AA$ lines respectively; however their abundances are confirmed by checking for good fits to O II blends at $4070 \AA$, 4317-4319 $\AA$ and $4650 \AA$, the O II lines at $4590 \AA, 4596 \AA$ and $4661 \AA$ and the $\mathrm{C}$ II doublet at $6578,6582 \AA$. The errors on constraining the $\mathrm{CNO}$ abundances using this method were typically up to $\sim 0.3$ dex.

\subsection{Determination of stellar wind properties}

The stellar wind parameters $\dot{M}, \beta$ and $v_{\text {turb }}$ were then constrained using the usual method outlined below (again the same procedure used by Hillier et al. 2003; Crowther et al. 2006; Bouret et al. 2003; Martins et al. 2005). CMFGEN allows for a treatment of turbulence in the stellar wind by assuming a radiallydependent microturbulent velocity, defined as

$v_{\text {turb }}=v_{\min }+\frac{\left(v_{\max }-v_{\min }\right) v(r)}{v_{\infty}}$

where $v_{\min }$ is the minimum turbulent velocity occurring in the photosphere and $v_{\max }$ is the maximum turbulent velocity. Hillier et al. (2003) found that varying the turbulent velocity had little effect on the temperature structure calculated by CMFGEN. In this work, values of $v_{\min }=10 \mathrm{~km} \mathrm{~s}^{-1}$ and $v_{\max }=50 \mathrm{~km} \mathrm{~s}^{-1}$ are adopted as limits. Typically $v$ reaches the value of $v_{\text {turb }}$ around $r=1.05 R_{*}$.

1. values for the mass loss rate of each star were constrained by fits to the $\mathrm{H} \alpha$ profile, with each fit aiming to reproduce the overall profile shape and amplitude;

2. values of $v_{\infty}$ are best determined from the UV so values obtained through UV line synthesis modelling (see Prinja et al. 2005; Paper II) are used here;

3 . the value of $\beta$ is also varied in order to improve the shape of the model profile fit with respect to the observed profile, but this has no effect for $\mathrm{H} \alpha$ profiles in absorption, in which case values obtained from SEI analysis were used;

4. estimates of the microturbulent velocity, $v_{\text {turb }}$, were then made by fitting the Si III 4552, 4568 and 4575 lines;

5. the model fit to the temperature and luminosity sensitive lines was checked after altering these parameters to ensure consistency.

The sample of $20 \mathrm{~B}$ supergiants showed some variation in overall profile shape, with four stars showing $\mathrm{H} \alpha$ in emission, six in absorption and the remainder displaying a more complex morphology. In the last case, the profiles are partly in absorption with some emission component also detectable, implying that the profile has been filled in by stellar wind emission. The B2-B5 supergiants display $\mathrm{H} \alpha$ profiles with a $\mathrm{P}$ Cygni profile shape, which implies that line scattering is playing a significant role in the line's formation, though this is not normally observed until late $\mathrm{B} / \mathrm{A}$ supergiants. The majority of stars in this sample have no record of $\mathrm{H} \alpha$ variability so that any changes in the line profile morphology and amplitude can be considered negligible for the purpose of this analysis. However $\epsilon$ Ori, HD 13854, HD 14818 and HD 42087 all display significant $\mathrm{H} \alpha$ variability according to Morel et al. (2004). In view of this problem, for $\epsilon$ Ori we have assumed a radio mass loss rate, $\dot{M}_{\text {radio }}$, of $1.9 \times 10^{-6} M_{\odot} \mathrm{yr}^{-1}$, as measured by Blomme et al. (2002), thereby avoiding the inaccuracies involved in deriving $\dot{M}$ from a variable $\mathrm{H} \alpha$ line. An estimate of the error in fitting the $\mathrm{H} \alpha$ profile of this star is given nonetheless is Table 8. Unfortunately this approach is not possible for the other 3 stars since there are no reliable $\dot{M}_{\text {radio }}$ values available in the literature. The problems associated with deriving $\dot{M}$ from the $\mathrm{H} \alpha$ profiles of these stars are discussed in the next section.

\section{Results}

\subsection{The $B$ supergiant $T_{\text {eff }}$ scale}

Model fits to the optical spectra of HD 192660 (B0 Ib), HD 213087 (B0.5 Ia), HD 13854 (B1 Iab), HD 193183 (B1.5 Ib), HD 14818 (B2 Ia), HD 198478 (B2.5 Ia), HD 53138 (B3 Ia) and HD 58350 (B5 Ia) are shown in Fig. 2 (4050-4250 Å), Fig. 3 (4250-4450 ̊) and Fig. 4 (4450-4650 A). Overall CMFGEN has succeeded in providing excellent fits to the observed spectrum of each star. The models succeed in reproducing the $\mathrm{H}, \mathrm{He}, \mathrm{Si}$ and $\mathrm{Mg}$ lines quite accurately. However, some individual spectral lines are more difficult to model than others. The Si IV $4089 \AA$ line is sometimes underestimated in B0-B2 supergiants, with the effect being most pronounced in B1-B2 supergiants, which might be partly due to a blend with O II. It is also noticeable that the model Si IV line displays a slight sensitivity to mass loss. Hillier et al. (2003) also noted that some model photospheric lines can be affected by mass loss and more importantly, Dufton et al. (2005) noted when using FASTWIND that Si IV $4116 \AA$ and the Si III multiplet $4552,4568,4575 \AA$ were affected by the stellar wind. However, whilst using CMFGEN, we have not observed any significant stellar wind effects on the Si III multiplet. The values of $T_{\text {eff }}$ derived for these stars can still be justified since the model spectrum still fits the rest of the spectrum, including the Si III, Mg II and He I lines, very well. In the cases where the model does underestimate the Si IV $4089 \AA$ line, the use of a model with a higher $T_{\text {eff }}$ that provided a better fit to the Si IV line would provide a worse fit to the rest of the observed spectrum. Note that the values of $T_{\text {eff }}$ obtained in these cases were still derived using the silicon ionisation balance and the effect of the compromise attained between fitting the Si IV line underestimated by the model and the rest of the spectrum is reflected in the value of $\Delta T_{\text {eff }}$ quoted in Table 3 . It is also intriguing to note that CMFGEN predicts two absorption lines at $4163 \AA$ (see in B1.5-B5 supergiants) and $4168.5 \AA$ (seen in all B0-B5 supergiants) that are not observed in any of the sample stars. These predicted lines also appear in the CMFGEN models of Crowther et al. (2006), where it appears that they have identified the line at $4168.5 \AA$ as He I but no explanation is given for the line at $4163 \AA$. We can confirm the identify of the line at $4168.5 \AA$ and also add that the line at $4163 \AA$ is Fe III.

The values of $T_{\text {eff }}, \log g, \log \left(L / L_{\odot}\right), E(B-V)$ and $M_{V}$ derived for each of the $20 \mathrm{~B}$ supergiants in the sample are listed in Table 3. These results show that $\mathrm{B} 0-\mathrm{B} 5$ supergiants have a range in $T_{\text {eff }}$ of $14500-30000 \mathrm{~K}$, in $\log \left(L / L_{\odot}\right)$ of $4.30-5.74$ and that their stellar radii vary from about $20-71 R_{\odot}$. They also exhibit a range of $-6.04 \leq M_{V} \leq-7.26$ in brightness, confirming their status as some of the brightest stars in our Galaxy. The temperature scale for B supergiants derived here is shown in Fig. 5, plotted against spectral type. A drop of up to $10000 \mathrm{~K}$ in temperature is witnessed between $\mathrm{B} 0-\mathrm{B} 1$, whereas at lower spectral types, the $T_{\text {eff }}$ scale shows a more 


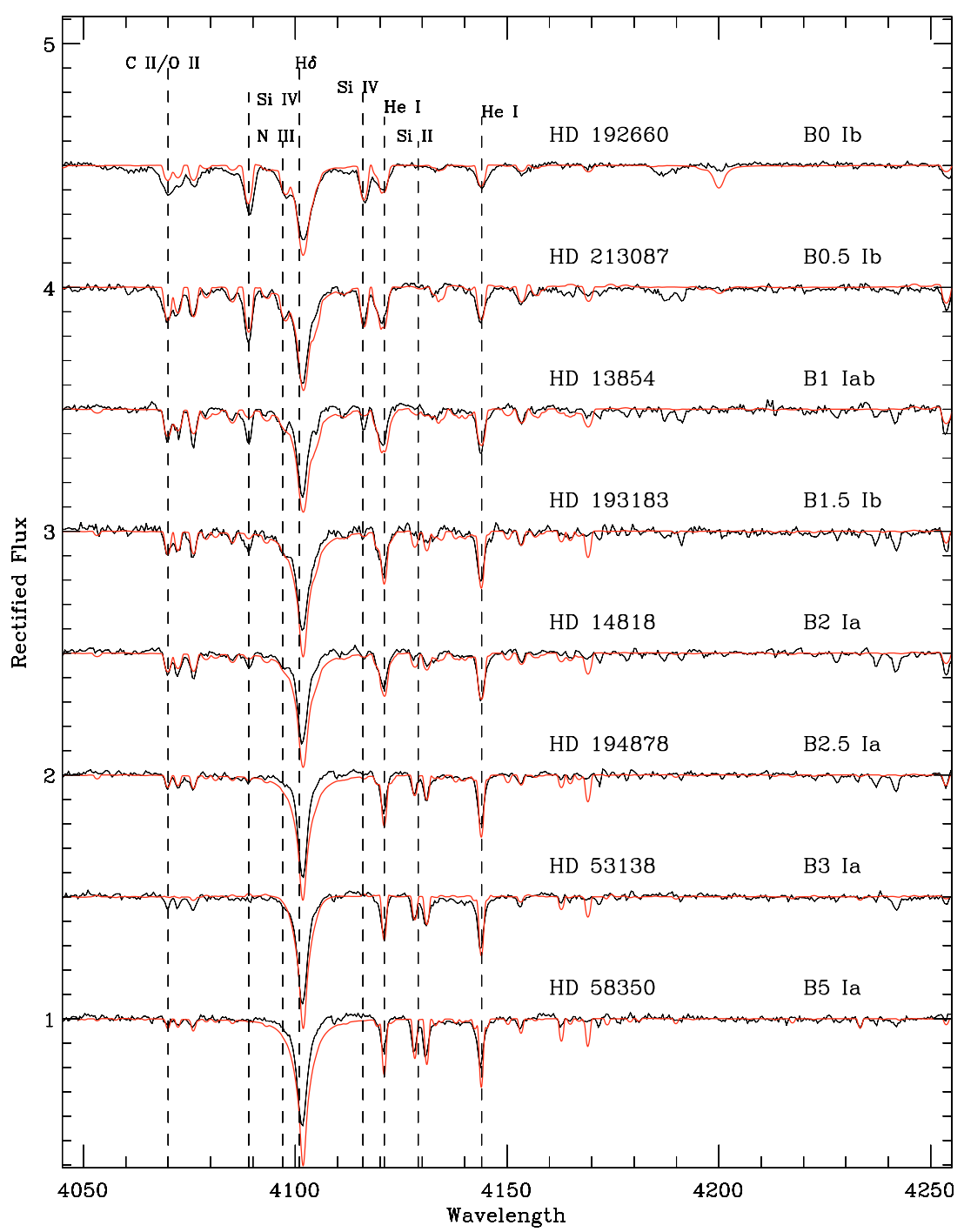

Fig. 2. Overall CMFGEN fit to the optical spectrum of B0-B5 supergiants (4050-4250 $)$. The solid black line is the observed spectrum and the red line denotes the CMFGEN model fit.

gradual decrease in $T_{\text {eff. }}$ The Galactic $\mathrm{O}$ star $T_{\text {eff }}$ scale published by Repolust et al. (2004) ranges from an $\mathrm{O} 2$ If star with $T_{\mathrm{eff}}=$ $42500 \mathrm{~K}$ down to an $09.5 \mathrm{Ia}$ star with $T_{\text {eff }}=29000 \mathrm{~K}$ and an O9.5 Ib star with $T_{\text {eff }}=32000 \mathrm{~K}$, meaning that the B supergiant $T_{\text {eff }}$ scale presented here carries on smoothly from the Galactic O supergiant $T_{\text {eff }}$ scale. Similarly the B supergiant $T_{\text {eff }}$ scale ends with B5 Ib stars having $T_{\text {eff }} \approx 15000 \mathrm{~K}$ and the Galactic A supergiant $T_{\text {eff }}$ scale derived by Venn (1995) begins with a $T_{\text {eff }}$ of $9950 \mathrm{~K}$. A gap between the B and A supergiant $T_{\text {eff }}$ scales is expected since none of the recently published B star $T_{\text {eff }}$ scales include B6-9 stars. The B supergiant temperature scale derived here also demonstrates the difference in $T_{\text {eff }}$ between B Ia and $\mathrm{B} \mathrm{Ib} / \mathrm{Iab} / \mathrm{II}$ stars. $\mathrm{B} 0-\mathrm{B} 2 \mathrm{Ib} / \mathrm{Iab}$ stars are found to be up to $2500 \mathrm{~K}$ hotter than B0-B2 Ia stars, with the exception of the stars HD 190603 (B1.5 Ia+) and HD 193183 (B1.5 Ib). However, a less significant difference of $500 \mathrm{~K}$ in $T_{\text {eff }}$ is found between B2-B5 Ia and B2-B5 Ib/II stars, with the B Ib stars again being hotter than their more luminous counterparts; this discrepancy is well within the margins of error in deriving $T_{\text {eff }}$ as typically $\Delta T_{\text {eff }}=500-1000 \mathrm{~K}$. We have compared our Galactic B supergiant $T_{\text {eff }}$ scale to other published values (Trundle et al. 2004; Trundle \& Lennon 2005; McErLean et al. 1999; Crowther et al. 2006) in Table 4. Where each author has several stars with the same spectral type, the values are averaged and marked with an asterisk in the table. Note that the results of McErLean et al. (1999) were obtained with an unblanketed stellar atmosphere code. If we compare our derived $T_{\text {eff }}$ values with those of the unblanketed McErLean et al. (1999) $T_{\text {eff }}$ scale, the use of a stellar-atmosphere code with a full treatment of line blanketing has the effect of lowering $T_{\text {eff }}$ by $1000-3000 \mathrm{~K}$ for Galactic $\mathrm{B}$ supergiants. This is not as drastic as the reduction found for O supergiants, which can be as high as $7000 \mathrm{~K}$ for extreme stars (Crowther et al. 2002). If we compare our derived $T_{\text {eff's to }}$ those of McErLean et al. (1999), with whom we have 10 target stars in common (HD 37128, HD 38771, HD 2905, HD 13854, HD 193183, HD 14818, HD 206165, HD 42087 and HD 53138), we find reasonably good agreement except for B1 Ia/Iabs, where the McErLean et al. (1999) results imply that a B1 supergiant is $2500-3000 \mathrm{~K}$ hotter than our values. The SMC B supergiant temperature scale (Trundle et al. 2004; Trundle \& Lennon 2005) also implies a much hotter B1 supergiant, but it is expected that SMC stars will be hotter than Galactic stars (see e.g. the O star temperature scales of Massey et al. (2005) (SMC) and Repolust et al. (2004) (Galactic) where the SMC stars are up to $4000 \mathrm{~K}$ hotter than the Galactic ones). 


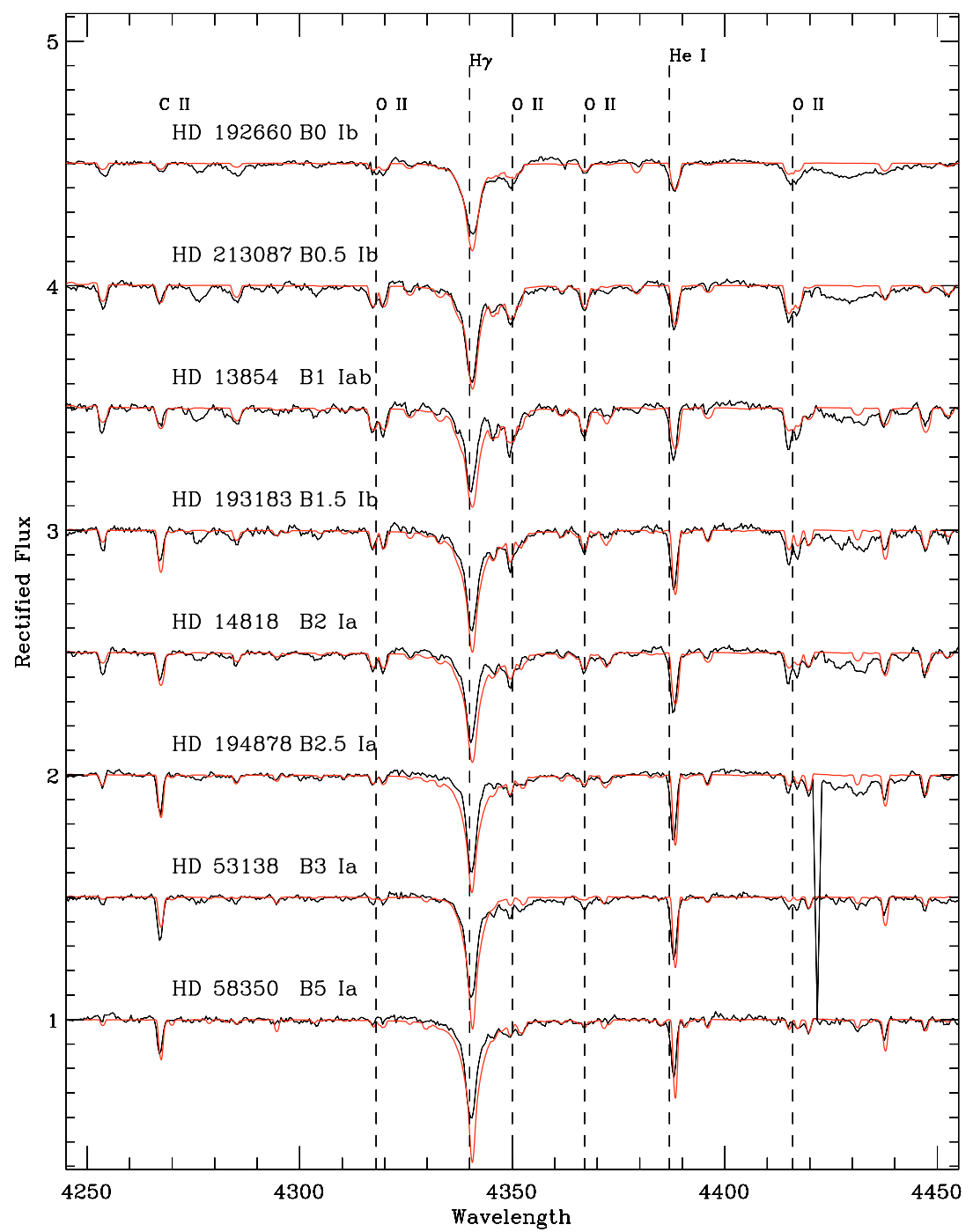

Fig. 3. Overall CMFGEN fit to the optical spectrum of B0-B5 supergiants $(4200-4450 \AA)$. The solid black line is the observed spectrum and the red line denotes the CMFGEN model fit.

\subsection{Log g estimates}

An example of a TLUSTY $\log g$ fit to the $\mathrm{H} \gamma$ profile of HD 164353 is shown in Fig. 7. Fits to the $\mathrm{H} \gamma$ and $\mathrm{H} \delta$ profiles of all $20 \mathrm{~B}$ supergiants can be found online. TLUSTY is a purely photospheric code therefore the model Balmer lines are also photospheric; however in B supergiants the observed Balmer lines suffer from wind contamination, where hydrogen photons emitted in the wind at the same wavelengths as $\mathrm{H} \gamma$ and $\mathrm{H} \delta$ "fill in" the absorption profile. This makes it appear more "shallow" when compared to a photospheric profile and explains the difference in depth between the two profiles shown in Fig. 7. Values of $\log g$ derived from $\mathrm{H} \gamma$ and $\mathrm{H} \delta$ were in good agreement in general and no discrepancies larger than the margin of error on $\log g$ were found. Only small discrepancies were found for B0-B0.5 stars where a model with a $\log g$ value 0.13 dex higher than the adopted value might provide a slight better fit to the $\mathrm{H} \delta$ profile. However this ambiguity can be attributed to the influence of a large $\mathrm{N}$ III blend on the blue wing of $\mathrm{H} \delta$ masking where the actual wing of the profile should really lie. In these cases a very good fit is made to $\mathrm{H} \gamma$ so the value derived from $\mathrm{H} \gamma$ is taken. Some difficulties were encountered when trying to fit the $\mathrm{H} \gamma$ and $\mathrm{H} \delta$ profiles of HD 192660, HD 64760, HD 190603, HD 13854 \& HD 190066 due to the observed asymmetry of the $\mathrm{H} \gamma$ and
$\mathrm{H} \delta$ profiles. This is particularly evident in the HD 190603, a B1.5 Ia hypergiant with a strong wind evident from the P Cygni shape of its $\mathrm{H} \beta$ profile. The $T_{\text {eff }}-\log g$ scale derived from this work is shown in Fig. 6, where higher $\log g$ values are found for B Ib stars. The $\log g$ values derived for B Ia stars are 0.1-0.2 dex higher than those obtained by Kudritzki et al. (1999); Crowther et al. (2006) for a sample of Galactic B supergiants, whereas the Trundle et al. (2004); Trundle \& Lennon (2005) values for SMC B supergiants are generally higher than those for Galactic B supergiants.

\subsection{Stellar masses and the mass discrepancy}

Using our estimates of $\log g$, spectroscopic masses have been derived for each of the $20 \mathrm{~B}$ supergiants and imply a range of $8 \leq M_{*} \leq 52$. Estimates of the evolutionary mass, $M_{\text {evol }}$, were then obtained using our derived stellar parameters and the stellar evolutionary tracks of Meynet \& Maeder (2000). The positions of our 20 Galactic B supergiants on the Hertzsprung-Russell diagram, along with other Galactic B supergiants (Crowther et al. 2006), SMC B supergiants (Trundle et al. 2004; Trundle \& Lennon 2005) and Galactic O stars (Repolust et al. 2004), are shown in Fig. 8. Here, the Meynet \& Maeder (2000) stellar 


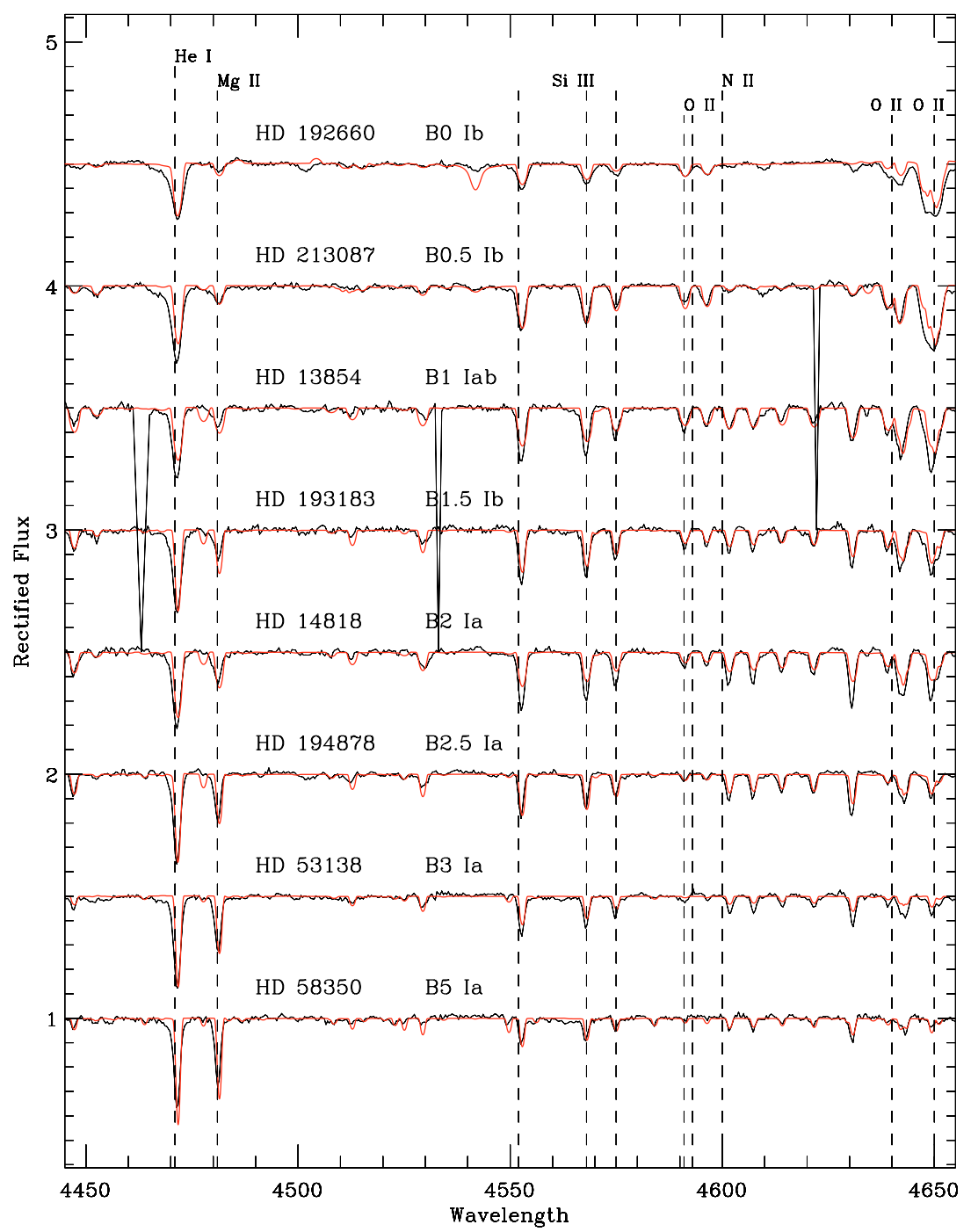

Fig. 4. Overall CMFGEN fit to the optical spectra of B0-B5 supergiants (4450-4650 $\AA$ ). The solid black line is the observed spectrum and the red line denotes the CMFGEN model fit.

evolutionary tracks have been used, which include the effects of rotation and are therefore more appropriate for OB supergiants. In order to demonstrate the effect of different stellar parameters on a star's precise position on the HR diagram, Galactic B supergiants common to both our sample and that of Crowther et al. (2006) are joined by a dotted line. A comparison of both masses is shown in Fig. 9. For 14 out of the 20 B supergiants, $M_{\text {evol }}>M_{\text {spec }}$ as found by Herrero et al. (2002). However, for the 5 other stars, which (excluding the rapid rotator HD 64760) have $\log \left(L / L_{\odot}\right) \geq 5.54, M_{\text {evol }}<M_{\text {spec. }}$. The dependence of the mass discrepancy with luminosity is examined further in Fig. 10 and compared to the mass discrepancy for SMC B supergiants investigated by Trundle et al. (2004); Trundle \& Lennon (2005). Both data sets exhibit a peak in the mass discrepancy at $5.4 \leq \log \left(L / L_{\odot}\right) \leq 5.5$ that drops off quite rapidly.

\subsection{Calibration of $B$ supergiant fundamental parameters}

A calibration of stellar atmosphere parameters (i.e., $T_{\text {eff }}, \log L_{*}$, $\log g, M_{*}, R_{*}$ ) according to spectral type has been carried out, using the fundamental parameters derived for our sample and that of Crowther et al. (2006). A linear regression was applied to the trend of $T_{\text {eff }}$ with spectral type; once the $T_{\text {eff }}$ scale had been established, linear regressions were made to the trends of log $T_{\text {eff }}$ vs. $\log L_{*}$ and $\log T_{\text {eff }}$ vs. $\log g$, from which the values of $R_{*}$ and $M_{*}$ were then calculated. The resulting values of $T_{\mathrm{eff}}, \log L_{*}$, $\log g, M_{*}$ and $R_{*}$ for each spectral type are shown in Table 5.

\subsection{Evidence for CNO processing in B supergiants}

As previously mentioned, it was Walborn (1976) who first suggested that the nitrogen and carbon anomalies found in OB stars can be explained by their evolutionary status, with OBC stars being the least evolved. It therefore follows that a typical OB supergiant should display some partial CNO processing, in the form of nitrogen enrichment accompanied by CO depletion. Several authors (Trundle et al. 2004; Trundle \& Lennon 2005; Evans et al. 2004a; Venn 1995) have found N enrichments and CO depletions in OBA stars with respect to solar abundances. All 20 B supergiants in our sample show evidence for partial CNO processing in their spectra. The details of the $\mathrm{CNO}$ abundances derived for individual stars are given in Table 6.

The majority of Galactic B supergiants show a modest nitrogen enrichment, but some stars (HD 37128, HD 192660 and HD 191243) are slightly nitrogen deficient. Walborn (1976) observed that Orion belt stars such as HD 37128 ( $\epsilon$ Ori) are 
Table 3. Fundamental parameters $\left(T_{\text {eff }}, \log g, \log \left(L / L_{\odot}\right), R_{*}\left(R_{\odot}\right), E(B-V) M_{V}\right.$ and $\left.M_{\text {evol }}\right)$ derived for the sample of 20 Galactic B supergiants. Values of $v_{\mathrm{e}} \sin i$ are taken from Howarth et al. (1997) and are expressed in $\mathrm{km} \mathrm{s}^{-1}$.

\begin{tabular}{|c|c|c|c|c|c|c|c|c|c|}
\hline HD No. & Sp. type & $\bar{T}_{\text {eff }}(\mathrm{K})$ & $\overline{\log g}$ & $\overline{\log \left(L / L_{\odot}\right)}$ & $\overline{\overline{R_{*}\left(R_{\odot}\right)}}$ & 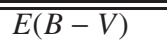 & $\overline{\overline{M_{V}}}$ & 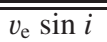 & $\overline{M_{\mathrm{evol}}}$ \\
\hline 37128 & B0 Ia & $27500 \pm 1000$ & 3.13 & $5.73 \pm 0.11$ & $32.4 \pm 0.75$ & $0.08 \pm 0.02$ & $-6.89 \pm 0.05$ & 91 & 40 \\
\hline 192660 & B0 Ib & $30000 \pm 1000$ & 3.25 & $5.74 \pm 0.13$ & $23.4 \pm 1.03$ & $0.80 \pm 0.10$ & $-6.66 \pm 0.10$ & 94 & 33 \\
\hline 204172 & B0.2 Ia & $28500 \pm 1000$ & 3.13 & $5.48 \pm 0.27$ & $22.4 \pm 3.23$ & $0.12 \pm 0.04$ & $-6.07 \pm 0.30$ & 87 & 41 \\
\hline 38771 & B0.5Ia & $26000 \pm 1000$ & 3.00 & $5.48 \pm 0.14$ & $27.0 \pm 1.24$ & $0.07 \pm 0.01$ & $-6.48 \pm 0.10$ & 91 & 33 \\
\hline 185859 & B0.5Ia & $26000 \pm 1000$ & 3.13 & $5.54 \pm 0.14$ & $29.1 \pm 1.34$ & $0.53 \pm 0.02$ & $-6.54 \pm 0.10$ & 74 & 35 \\
\hline 213087 & B0.5Ib & $27000 \pm 1000$ & 3.13 & $5.69 \pm 0.11$ & $32.0 \pm 0.01$ & $1.30 \pm 0.01$ & $-6.20 \pm 0.10$ & 88 & 40 \\
\hline 64760 & B $0.5 \mathrm{Ib}$ & $28000 \pm 2000$ & 3.38 & $5.48 \pm 0.26$ & $23.3 \pm 2.15$ & $0.15 \pm 0.05$ & $-6.36 \pm 0.20$ & 265 & 33 \\
\hline 2905 & BC0.7Ia & $23500 \pm 1500$ & 2.75 & $5.48 \pm 0.22$ & $33.0 \pm 1.52$ & $0.29 \pm 0.03$ & $-7.00 \pm 0.10$ & 91 & 33 \\
\hline 13854 & B1 Iab & $20000 \pm 2000$ & 2.50 & $5.54 \pm 0.57$ & $49.2 \pm 1.33$ & $0.60 \pm 0.10$ & $-6.41 \pm 0.50$ & 97 & 33 \\
\hline 190066 & B1 Iab & $21000 \pm 1000$ & 2.88 & $5.54 \pm 0.20$ & $41.4 \pm 1.89$ & $0.55 \pm 0.02$ & $-6.04 \pm 0.10$ & 82 & 33 \\
\hline 190603 & B1.5 Ia+ & $19500 \pm 1000$ & 2.38 & $5.41 \pm 0.23$ & $44.5 \pm 3.07$ & $0.70 \pm 0.05$ & $-6.85 \pm 0.15$ & 79 & 27 \\
\hline 193183 & B1.5 Ib & $18500 \pm 1000$ & 2.63 & $5.00 \pm 0.26$ & $30.8 \pm 2.84$ & $0.70 \pm 0.06$ & $-6.43 \pm 0.20$ & 68 & 18 \\
\hline 14818 & B2 Ia & $18000 \pm 500$ & 2.38 & $5.40 \pm 0.27$ & $51.4 \pm 7.10$ & $0.62 \pm 0.10$ & $-6.70 \pm 0.30$ & 82 & 26 \\
\hline 206165 & B2 Ib & $18000 \pm 500$ & 2.50 & $5.18 \pm 0.26$ & $39.8 \pm 5.50$ & $0.56 \pm 0.10$ & $-6.64 \pm 0.30$ & 73 & 21 \\
\hline 198478 & B2.5 Ia & $17500 \pm 500$ & 2.25 & $5.26 \pm 0.14$ & $46.1 \pm 2.12$ & $0.40 \pm 0.05$ & $-7.26 \pm 0.10$ & 61 & 23 \\
\hline 42087 & B2.5 Ib & $18000 \pm 1000$ & 2.50 & $5.11 \pm 0.24$ & $36.6 \pm 1.69$ & $0.60 \pm 0.02$ & $-6.11 \pm 0.10$ & 71 & 21 \\
\hline 53138 & B3 Ia & $16500 \pm 500$ & 2.25 & $5.30 \pm 0.27$ & $54.7 \pm 7.56$ & $0.35 \pm 0.10$ & $-6.79 \pm 0.30$ & 58 & 23 \\
\hline 58350 & B5 Ia & $15000 \pm 500$ & 2.13 & $5.18 \pm 0.17$ & $57.3 \pm 2.64$ & $0.05 \pm 0.04$ & $-7.12 \pm 0.10$ & 50 & 21 \\
\hline 164353 & B5 Ib & $15500 \pm 1000$ & 2.75 & $4.30 \pm 1.30$ & $19.6 \pm 8.05$ & $0.71 \pm 0.05$ & $-6.15 \pm 2.00$ & 44 & 10 \\
\hline 191243 & B5 Ib & $14500 \pm 1000$ & 2.75 & $5.30 \pm 0.37$ & $70.8 \pm 3.26$ & $0.93 \pm 0.03$ & $-6.59 \pm 0.10$ & 38 & 21 \\
\hline
\end{tabular}

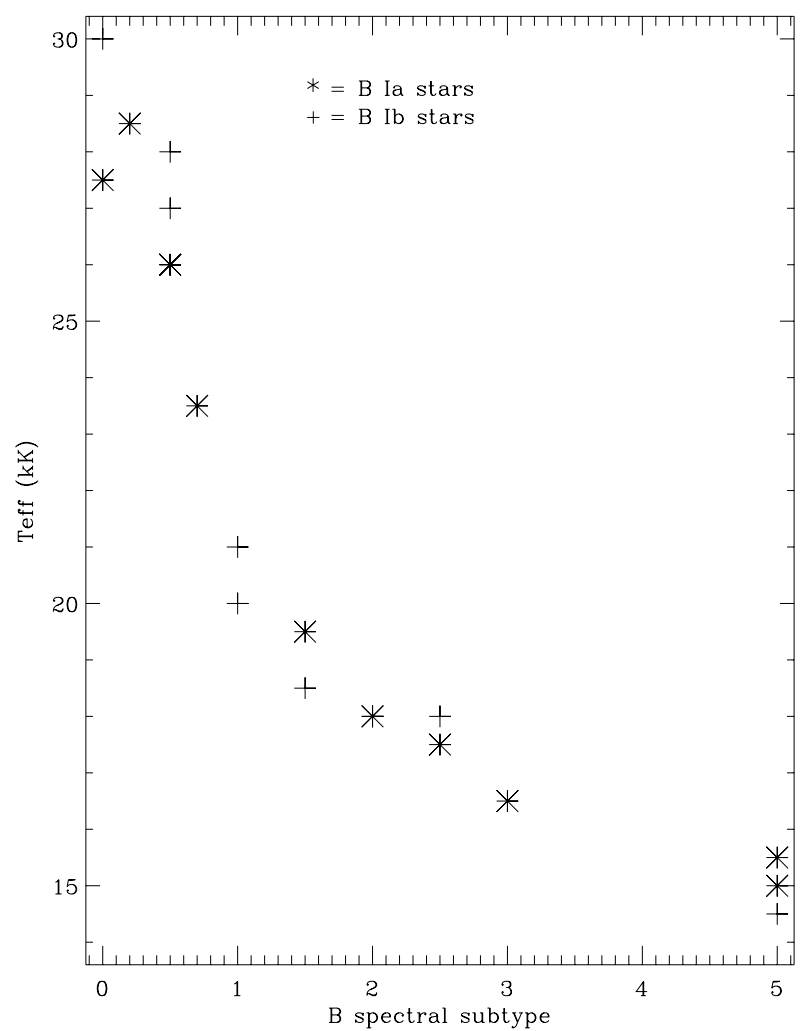

Fig. 5. The Galactic B supergiant $T_{\text {eff }}$ scale as a function of spectral type. B Ia stars are indicated by an asterisk, whilst B Ib stars are marked by a plus sign.

nitrogen deficient due to the weakness of the N III $4097 \AA$ and $4640 \AA$ (blend) spectral lines. The largest nitrogen enhancements are seen for B1-B2 stars (HD 13854, HD 190603 and HD 14818). It is of interest to note that Walborn (1976) classed HD 13854 as a morphologically normal B supergiant (as well as HD 38771), whereas we have found a modest yet significant $\mathrm{N}$ enrichment in this star.

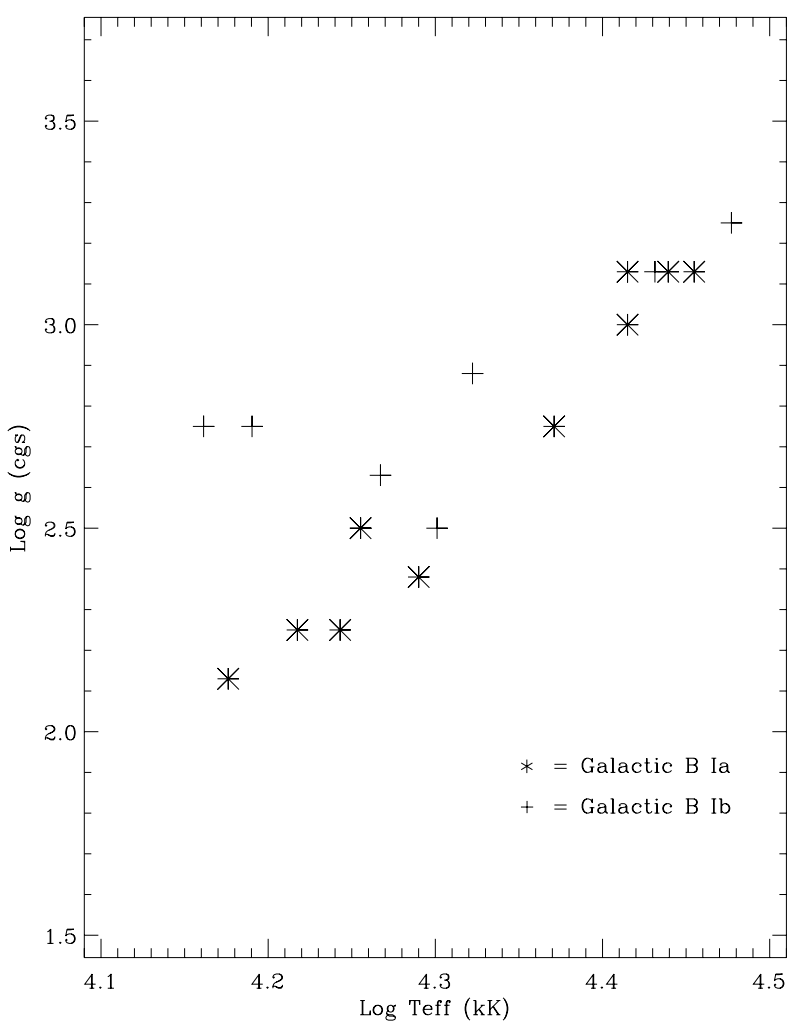

Fig. 6. The Galactic B supergiant $T_{\text {eff }}-\log g$ scale.

In general, the fits to the $\mathrm{CNO}$ diagnostic lines are good. On the whole, nitrogen abundances constrained from N II $3995 \AA$ and N III $4097 \AA$ are in good agreement; the only exceptions being HD 37128 and HD 193183. It is well-documented in the literature that there exists a discrepancy between carbon abundances derived from the C II 4267.02, 4267.27 $\AA$ multiplet line and the C II multiplets at 6578 and $6582 \AA$, due to their strong sensitivity to nLTE effects and the adopted stellar parameters (see e.g., Nieva \& Przybilla 2006). A combination of high-resolution and high signal-to-noise spectra, along with 
Table 4. Values of $T_{\text {eff }}$ (expressed in terms of $10^{3} \mathrm{~K}$ ) obtained in this thesis work and from Trundle et al. (2004); Trundle \& Lennon (2005); Kudritzki et al. (1999); McErLean et al. (1999). Values marked with an asterisk denote where values from one author have been averaged and are quoted to 1 decimal place. $†$ The B0.5 Ib star HD 64760 has been omitted here because it is a rapid rotator.

\begin{tabular}{lllll}
\hline \hline Sp type & This work & Trundle & McErlean & Crowther \\
\hline B0 Ia & 27.5 & $27.0^{*}$ & 28.5 & $27.4^{*}$ \\
B0 Ib & 30.0 & - & - & - \\
B0.2 Ia & 28.5 & - & 28.5 & - \\
B0.5 Ia & 26.0 & $27.3^{*}$ & 27.5 & $26.0^{*}$ \\
B0.5 Ib & $27.0 \dagger$ & - & $26.5^{*}$ & - \\
B0.7 Ia & 23.5 & - & 24.0 & 22.9 \\
B1 Ia & - & $23.8^{*}$ & - & 22.0 \\
B1 Iab/Ib & $20.5^{*}$ & - & $23.3^{*}$ & $21.8^{*}$ \\
B1.5 Ia & 19.5 & $21.3^{*}$ & 21.25 & 18.17 \\
B1.5 Ib & 18.5 & - & $22.3^{*}$ & - \\
B2 Ia & 18.0 & 19.0 & 19.83 & $18.6^{*}$ \\
B2 Ib & 18.0 & - & $20.8^{*}$ & - \\
B2.5 Ia & 17.5 & 16.5 & 18.0 & 16.5 \\
B2.5 Ib & 18.0 & - & 20.5 & - \\
B3 Ia & 16.5 & 14.0 & $17.9^{*}$ & $15.8^{*}$ \\
B4 Iab & - & - & 16.5 & - \\
B5 Ia & 15.0 & $14.5^{*}$ & $15.4^{*}$ & - \\
B5 Ib/II & $15.0^{*}$ & - & $15.8^{*}$ & - \\
\hline
\end{tabular}

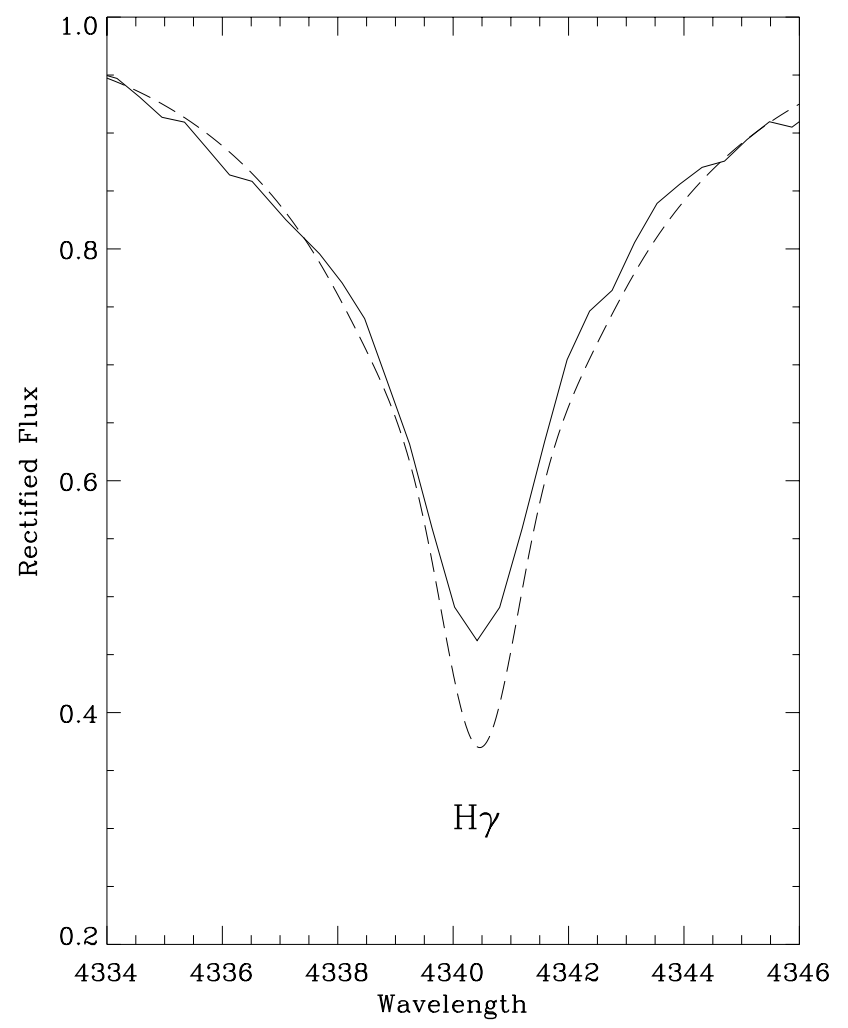

Fig. 7. Example of a TLUSTY $\log g$ fit to $\mathrm{H} \gamma$ profile of the $\mathrm{B} 5 \mathrm{Ib} / \mathrm{II}$ star HD 164353. A value of $\log g=2.75$ is used here.

sufficiently- detailed model atoms, are required to attempt to resolve this problem. It is unlikely that our data is of a suitable resolution and signal-to-noise to attempt to solve this discrepancy, but we will nonetheless discuss our findings as appropriate. For our sample of B supergiants, the C II multiplets at 6578 and $6582 \AA$ are not prominent for B0-B1 supergiants; however for B1-B5 stars the lines are distinguishable. The fits to the C II 4267.02, 4267.27 $\AA$ multiplet are very good but CMFGEN

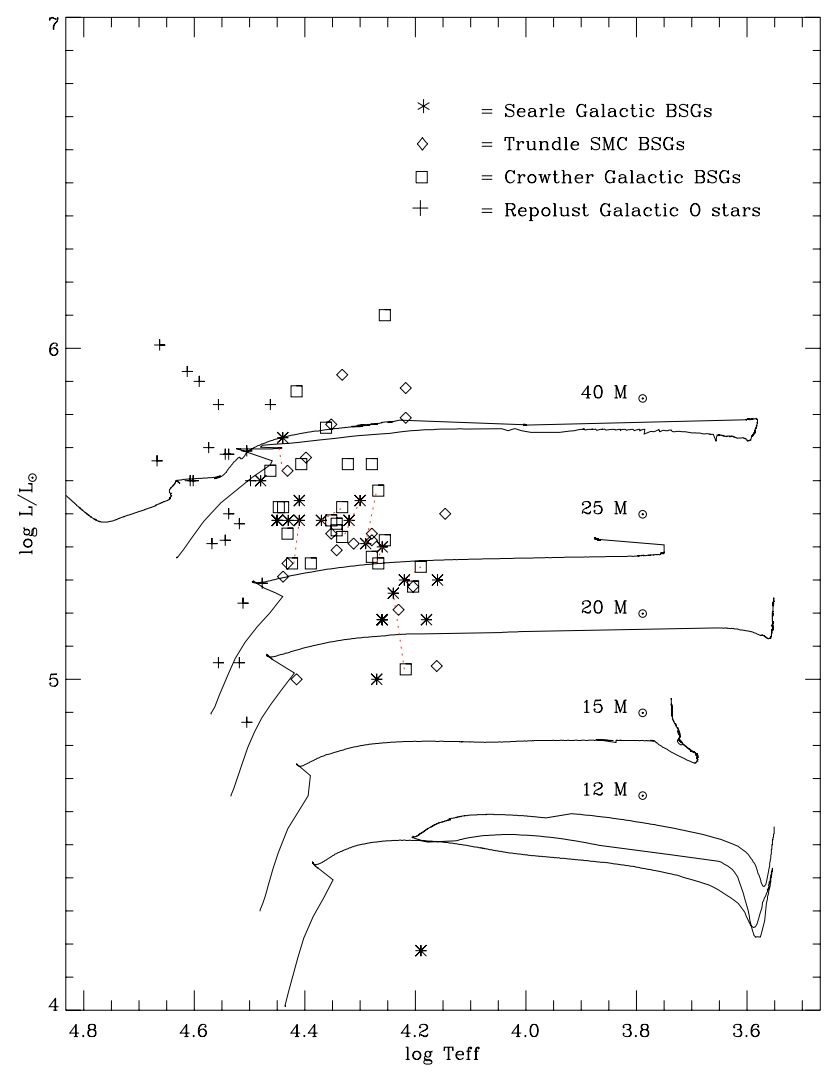

Fig. 8. Position of the sample of Galactic B supergiants on the Hertzsprung-Russell diagram, along with other Galactic B supergiants Crowther et al. (2006), SMC B supergiants Trundle et al. (2004); Trundle \& Lennon (2005) and Galactic O stars Repolust et al. (2004). Evolutionary tracks are taken from Maeder \& Meynet (2001) and imply $15 M_{\odot}<M_{\mathrm{evol}} \leq 40 M_{\odot}$ for the sample of 20 Galactic B supergiants presented in this work. Galactic B supergiants that are common to both our sample and that of Crowther et al. (2006) are joined by a dotted red line to illustrate the effect of different stellar parameters on a star's precise location on the HR diagram.

tends to overestimate the C II 6578 and $6582 \AA$ multiplets. In the case of constraining the nitrogen abundances, in general good agreement is found between the abundance implied from the N III 4097 and N II 3995, 4447, $4630 \AA$ A. Some exceptions are found for some B0-B0.5 supergiants (HD 37128, HD 204172, HD 38771 and HD 192660) where very good fits are made to N II 3995 and $4447 \AA$, but N III 4097 and N II 4630 are both underestimated, with the model producing a much weaker N II 4447 line than observed. Evidently increasing the nitrogen abundance would then cause the N II 3995 and $4447 \AA$ lines to be overestimated. For HD 185859 and HD 213087, much better agreement is found between all four nitrogen diagnostics.

There is still an intriguing contradiction that $\kappa$ Cas, which has been defined as a carbon-rich star (Walborn 1976) has very similar CNO abundances to the stars HD 64760, HD 213087 which have not been noted as carbon rich by any other authors. The original criteria for classifying $\kappa$ Cas as a carbon-rich star were based on the weakness of its nitrogen lines as well as the strength of its carbon lines; this makes sense since (as Walborn 1976 explains) it is expected that nitrogen deficiency will be accompanied by carbon enrichment. In order to resolve this discrepancy, the IUE spectrum of $\kappa$ Cas has been compared to the IUE spectrum of the B0.7 Ia star HD 154090 (see Fig. 11). Looking at the C II $1324 \AA$ line, it is certainly no stronger than the same line in 


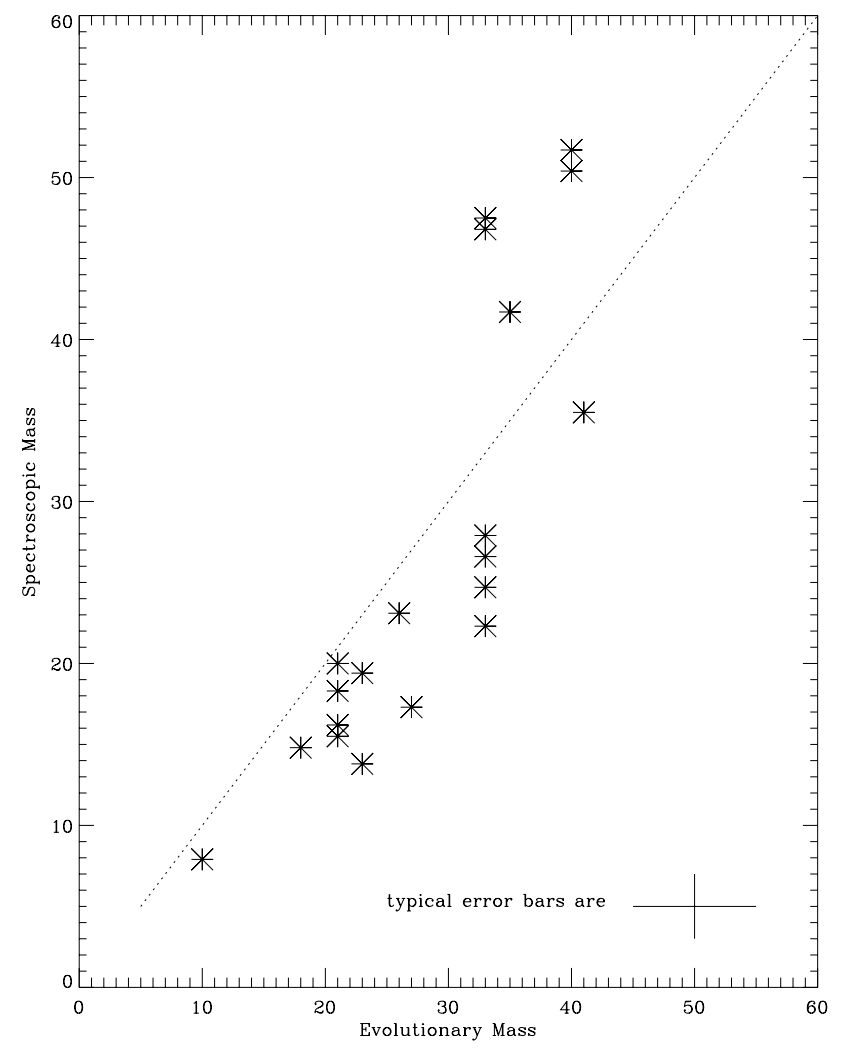

Fig. 9. Comparison of evolutionary and spectroscopically-derived stellar masses for the sample of B supergiants. The dotted line indicates 1:1 correspondance.

Table 5. Calibrations of fundamental parameters by spectral type for Galactic B supergiants, based on this work and that of Crowther et al. (2006).

\begin{tabular}{lccccc}
\hline \hline Sp. type & $T_{\text {eff }}$ & $\log \left(L / L_{\odot}\right)$ & $R_{*}\left(R_{\odot}\right)$ & $\log g$ & $M_{*}\left(M_{\odot}\right)$ \\
\hline B0 Ia & 28.1 & 5.60 & 26.9 & 2.99 & 25 \\
B0 Ib & 29.7 & 5.66 & 23.8 & 3.24 & 37 \\
B0.2 Ia & 26.7 & 5.62 & 30.4 & 3.04 & 36 \\
B0.2 Ib & 28.5 & 5.65 & 27.8 & 3.23 & 49 \\
B0.5 Ia & 24.7 & 5.58 & 33.8 & 2.90 & 33 \\
B0.5 Ib & 25.4 & 5.58 & 32.2 & 3.09 & 47 \\
B0.7 Ia & 23.6 & 5.53 & 35.1 & 2.72 & 23 \\
B0.7 Ib & 24.4 & 5.51 & 33.9 & 2.93 & 37 \\
B1 Ia & 22.0 & 5.44 & 36.5 & 2.41 & 12 \\
B1 Ib & 21.7 & 5.38 & 34.9 & 2.67 & 22 \\
B1.5 Ia & 19.9 & 5.44 & 44.5 & 2.41 & 18 \\
B1.5 Ib & 19.3 & 5.29 & 39.7 & 2.50 & 19 \\
B2 Ia & 18.3 & 5.41 & 51.0 & 2.32 & 19 \\
B2 Ib & 18.1 & 5.27 & 44.4 & 2.46 & 21 \\
B2.5 Ia & 17.2 & 5.39 & 56.5 & 2.24 & 19 \\
B2.5 Ib & 17.6 & 5.25 & 46.2 & 2.43 & 22 \\
B3 Ia & 16.4 & 5.37 & 60.4 & 2.16 & 19 \\
B3 Ib & 17.5 & 5.23 & 45.5 & 2.39 & 19 \\
B4 Ia & 15.8 & 5.34 & 63.5 & 2.06 & 16 \\
B4 Ib & 17.4 & 5.18 & 43.2 & 2.27 & 13 \\
B5 Ia & 15.7 & 5.33 & 63.0 & 2.03 & 15 \\
B5 Ib & 15.2 & 5.09 & 51.7 & 2.11 & 13 \\
\hline
\end{tabular}

the spectrum of HD 154090. The same is true of the C III line at $1247 \AA$. However, both stars appear to be nitrogen weak (see e.g., the N V wind resonance line around $1240 \AA$ ). Therefore on the basis of this evidence, it appears that the $\kappa$ Cas should be defined as a nitrogen weak star, rather than carbon rich.

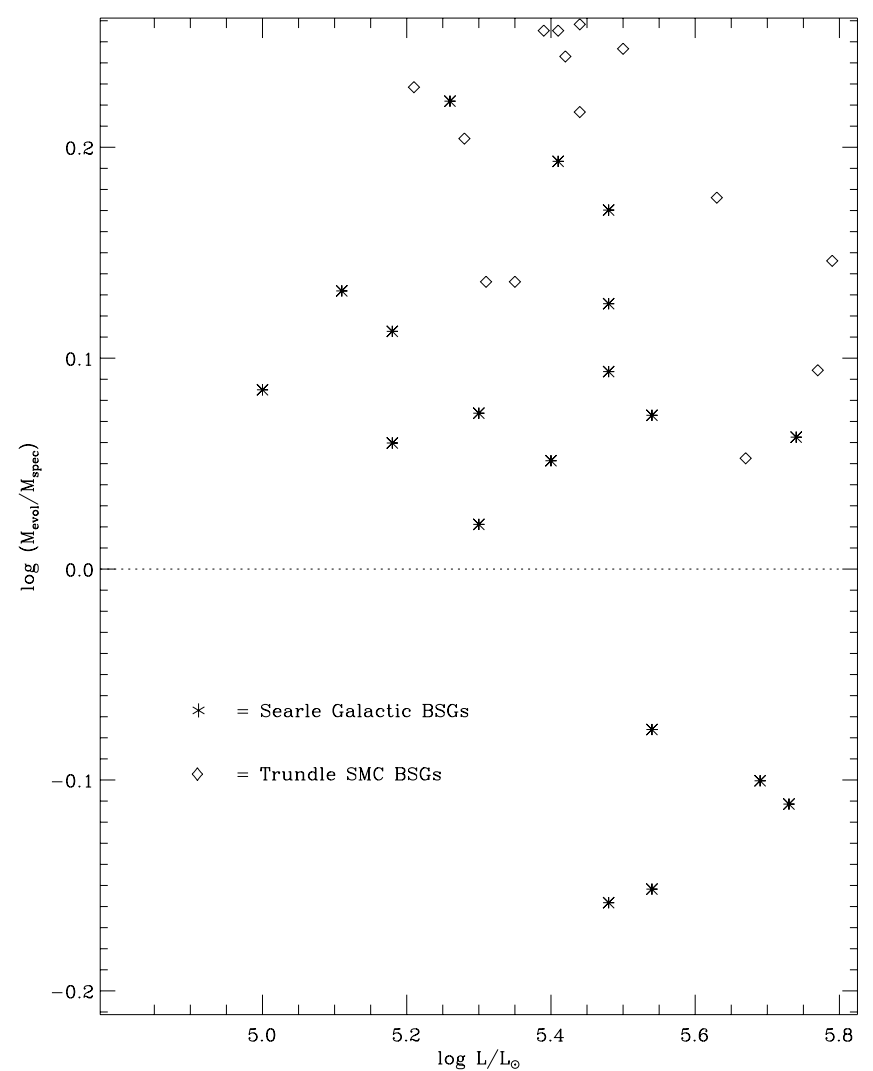

Fig. 10. Comparison of $\frac{M_{\text {evol }}}{M_{\text {spec }}}$ with luminosity (B5 Ib/II stars omitted). Values obtained for the 20 Galactic B supergiants (asterisks) are plotted with those derived for SMC B supergiants (diamonds) Trundle et al. (2004); Trundle \& Lennon (2005). The dotted line indicates $1: 1$ correspondance.

Crowther et al. (2006) found similar results for $\kappa$ Cas, citing it as having the "least nitrogen enriched abundance" in their sample as well as the lowest values for the $\mathrm{N} / \mathrm{C}$ and N/O ratios.

The CNO abundances derived here for the sample of $20 \mathrm{~B}$ supergiants are compared in Table 7 to values obtained by other authors (Trundle et al. 2004; Trundle \& Lennon 2005; Evans et al. 2004a; Crowther et al. 2006; Venn 1995, 1999) for OBA supergiants. The results from Trundle et al. (2004); Trundle \& Lennon (2005) SMC B supergiants have been combined to obtain mean $\mathrm{CNO}$ abundances based on a sample of 18 stars (but only 13 were used for the mean oxygen abundance since oxygen abundances were not derived for some B2.5-5 stars due to weak, unmeasurable O II lines). The data from Evans et al. (2004a) were purely based on CNO abundances derived from OB supergiants so that the results for nebular and $\mathrm{H}$ II regions included by the authors for comparison were omitted. It is clear from Table 7 that more CNO enrichment occurs in stars belonging to the Magellanic Clouds than Galactic stars. This is in accordance with Evans et al. (2004a), who found that OB supergiants in the LMC display a nitrogen enrichment that is greater than the nitrogen enrichments in Galactic B supergiants. Evans et al. (2004a) conclude that their sample of Magellanic Cloud stars show significant nitrogen enrichment due to efficient rotational mixing. The $\mathrm{CNO}$ abundances show no clear trend with effective temperature or $v_{\mathrm{e}} \sin i$. 
Table 6. Derived CNO abundances for the sample of Galactic B supergiants (expressed as $\log \left(\frac{N_{\mathrm{x}}}{N_{\mathrm{H}}}\right)+12$ ). The amount of nitrogen enrichment relative to carbon and oxygen respectively is given in the last two columns, calculated as $\log \left(\frac{N_{\mathrm{X}}}{N_{\mathrm{H}}}\right)_{*}-\log \left(\frac{N_{\mathrm{X}}}{N_{\mathrm{H}}}\right)_{\odot}$.

\begin{tabular}{lllllllll}
\hline \hline HD No. & Sp. type & $T_{\text {eff }}$ & $v_{\mathrm{e}} \sin i$ & $\mathrm{C}$ & $\mathrm{N}$ & $\mathrm{O}$ & $\mathrm{N} / \mathrm{C}$ & $\mathrm{N} / \mathrm{O}$ \\
SUN & G2 V & 5770 & - & 8.39 & 7.78 & 8.66 & -0.61 & -0.88 \\
\hline 37128 & B0 Ia & 27500 & 91 & 7.66 & 7.31 & 8.68 & +0.26 & -0.49 \\
192660 & B0 Ib & 30000 & 94 & 8.02 & 7.51 & 8.73 & +0.10 & -0.40 \\
204172 & B0.2 Ia & 28500 & 87 & 7.66 & 7.71 & 8.66 & +0.66 & -0.07 \\
38771 & B0.5 Ia & 26000 & 91 & 7.74 & 8.15 & 8.73 & +1.02 & +0.30 \\
185859 & B0.5 Ia & 26000 & 74 & 7.72 & 7.95 & 8.53 & +0.84 & +0.30 \\
213087 & B0.5 Ib & 27000 & 88 & 8.00 & 8.15 & 8.73 & +0.76 & +0.30 \\
64760 & B0.5 Ib & 28000 & 265 & 7.99 & 8.15 & 8.73 & +0.77 & +0.30 \\
2905 & BC0.7 Ia & 23500 & 91 & 7.99 & 8.16 & 8.80 & +0.78 & +0.24 \\
13854 & B1 Iab(e) & 20000 & 97 & 7.66 & 8.51 & 8.80 & +1.46 & +0.59 \\
190066 & B1 Iab(e) & 21000 & 82 & 7.88 & 8.15 & 8.53 & +0.88 & +0.50 \\
190603 & B1.5 Ia+ & 19500 & 79 & 7.48 & 8.76 & 8.73 & +1.89 & +0.91 \\
193183 & B1.5 Ib & 18500 & 68 & 7.66 & 8.15 & 8.73 & +1.10 & +0.30 \\
14818 & B2 Ia & 18000 & 82 & 7.66 & 8.72 & 8.90 & +1.67 & +0.70 \\
206165 & B2 Ib & 18000 & 73 & 7.96 & 8.15 & 8.43 & +0.80 & +0.60 \\
198478 & B2.5 Ia & 17500 & 61 & 7.86 & 8.29 & 8.45 & +1.04 & +0.72 \\
42087 & B2.5 Ib & 18000 & 71 & 7.76 & 8.11 & 8.80 & +0.96 & +0.19 \\
53138 & B3 Ia & 16500 & 58 & 7.78 & 8.32 & 8.60 & +1.15 & +0.60 \\
58350 & B5 Ia & 15000 & 50 & 7.78 & 8.29 & 8.75 & +1.12 & +0.42 \\
164353 & B5 Ib/II & 15500 & 44 & 7.78 & 7.89 & 8.53 & +0.72 & +0.24 \\
191243 & B5 Ib/II & 14500 & 38 & 7.70 & 7.65 & - & +0.56 & - \\
\hline
\end{tabular}

Table 7. Comparison of mean published CNO abundances for OBA supergiants (expressed as $\log \left(\frac{N_{\mathrm{x}}}{N_{\mathrm{H}}}\right)+12$ ).

\begin{tabular}{lllllll}
\hline \hline Author & Stellar group & $\mathrm{C}$ & $\mathrm{N}$ & $\mathrm{O}$ & $\mathrm{N} / \mathrm{C}$ & $\mathrm{N} / \mathrm{O}$ \\
\hline This work & Gal BSGs & 7.79 & 8.12 & 8.68 & +0.57 & +0.26 \\
Crowther et al. (2006) & Gal BSGs & 7.87 & 8.33 & 8.47 & +0.68 & +0.59 \\
Trundle et al. (2004, 2005) & SMC BSGs & 7.27 & 7.71 & 8.13 & +1.38 & 1.04 \\
Evans et al. (2004) & LMC OBSG & 7.49 & 8.55 & 8.02 & +1.28 & +1.26 \\
Evans et al. (2004) & SMC OBSG & 7.30 & 7.94 & 8.01 & +0.86 & +0.66 \\
Venn (1995) & Gal ASGs & 8.14 & 8.05 & - & +0.13 & - \\
Venn (1999) & SMC ASGs & - & 7.33 & 8.14 & - & -0.08 \\
\hline
\end{tabular}

\subsection{Mass loss rates for $B$ supergiants}

The mass loss rates obtained for this sample of 20 Galactic B supergiants are based on matches to the $\mathrm{H} \alpha$ profile and the resulting values are listed in Table 8 . All of these B supergiants have mass loss rates ranging between $-7.22 \leq \log \dot{M} \leq-5.30$, except the B5 II/Ib star HD 164353 for which $\dot{M}=6 \times 10^{-8}$ was derived. The errors quoted in Table 8 reflect the ambiguity involved in fitting $\mathrm{H} \alpha$ "by eye" (and therefore represent the maximum and minimum values of $\dot{M}$ that fit $\mathrm{H} \alpha$ reasonably) and are no greater than a factor of 2 . In some cases an upper or lower error limit only is quoted where the model fit over- or underestimates the observed $\mathrm{H} \alpha$ profile, meaning that a larger/smaller mass loss rate would not be appropriate. CMFGEN fits to the $\mathrm{H} \alpha$ profiles of $\kappa$ Cas, HD 190603, HD 14818, HD 190066, HD 193183 and HD 164353 are given in Fig. 12. In general, good fits are obtained for each star, but several difficulties have been encountered in trying to reproduce the observed $\mathrm{H} \alpha$ profiles. It is clear that all the observed profiles are asymmetric and it is likely that this is caused by the influence of resonant line scattering that is too weak to produce a "P Cygni"-type profile so merely results in a slightly asymmetric profile. In some stars e.g., HD 213087, it appears to be a redward emission component that partly fills in the profile, to such an extent that in some stars this red component is visible as a separate emission component (e.g., HD 206165) and the $\mathrm{H} \alpha$ profile begins to resemble a P Cygni profile (e.g., HD 14818). In the majority of stars, the peak/trough of the $\mathrm{H} \alpha$ profile has shifted from the line centre as observed in $\kappa$ Cas. This effect is particularly clear on comparing the $\mathrm{H} \alpha$ profile of $\kappa$ Cas with that of HD 190603, whose peak is much more central resulting in only a slight asymmetry to the overall profile. It is also of interest to note that CMFGEN predicts a "bump" in the blueward wing of the H $\alpha$ profile of HD 190603 that is not present in the observed profile; a similar phenomenon is observed for HD 193183.

A small, preliminary investigation into the effects of including clumping on the morphology of the model $\mathrm{H} \alpha$ profile was undertaken. Hillier \& Miller (1999) assume the winds are clumped with a volume filling factor $f$ and that no inter-clump medium is present. The volume filling factor, is defined as:

$f=f_{\infty}+\left(1.0-f_{\infty}\right) \mathrm{e}^{-\frac{v}{v_{\mathrm{cl}}}}$

where $f_{\infty}$ is the filling factor at $v_{\infty}$ and $v_{\mathrm{cl}}$ is the velocity at which clumping is "switched on" in the wind. However, to carry out a fair comparison between clumped and homogeneous models involves a larger parameter space than merely varying $f_{\infty}$ and $v_{\mathrm{cl}}$. For example, it is important to check for consistency in the atmospheric structure of both models i.e., that they sample the same optical depth in the photosphere and have the same density structures, which can include some fine tuning of the velocity law and the point at which the TLUSTY hydrostatic structure joins the CMFGEN density structure. It is also important to check that 


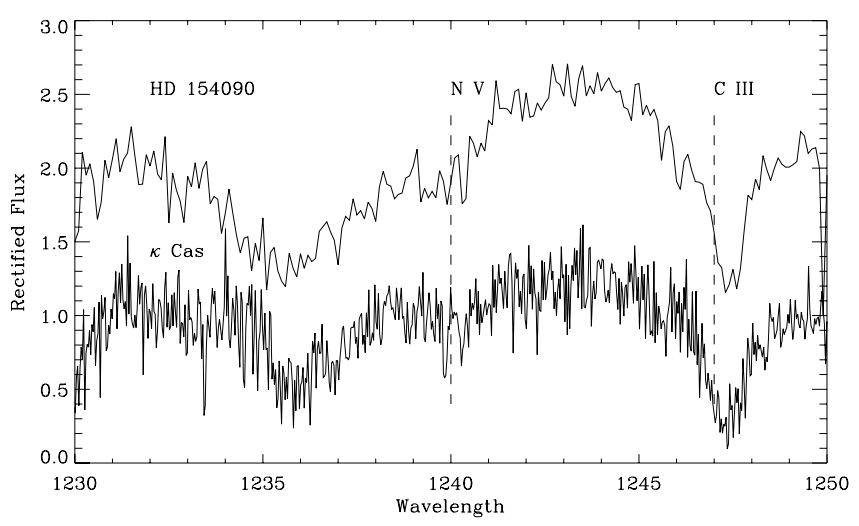

Table 8. Stellar wind parameters $\left(\dot{M}, \beta, v_{\infty}, v_{\text {turb }}\right)$ derived for a sample of 20 Galactic B supergiants. The errors given on $\dot{M}$ reflect the errors in fitting each individual $\mathrm{H} \alpha$ profile.

\begin{tabular}{llcccc}
\hline \hline HD No. & Sp type & $\dot{M}\left(10^{-6} M_{\odot} \mathrm{yr}^{-1}\right)$ & $\beta$ & $v_{\infty}\left(\mathrm{km} \mathrm{s}^{-1}\right)$ & $v_{\text {turb }}\left(\mathrm{km} \mathrm{s}^{-1}\right)$ \\
\hline 37128 & B0 Ia & $1.90_{-0.0}^{+0.9}$ & 1.1 & 1600 & 15 \\
192660 & B0 Ib & $5.00_{-0.0}^{+0.0}$ & 1.3 & 1850 & 20 \\
204172 & B0.2 Ia & $0.57_{-0.34}^{+0.7}$ & 1.0 & 1685 & 15 \\
38771 & B0.5 Ia & $1.20_{-0.2}^{+0.3}$ & 1.1 & 1390 & 15 \\
185859 & B0.5 Ia & $0.50_{-0.1}^{+0.1}$ & 1.5 & 1830 & 20 \\
213087 & B0.5 Ib & $0.70_{-0.0}^{+0.4}$ & 1.5 & 1520 & 20 \\
64760 & B0.5 Ib & $1.10_{-1.0}^{+1.0}$ & 1.0 & 1600 & 15 \\
2905 & BC0.7 Ia & $2.50_{-0.5}^{+0.0}$ & 1.5 & 850 & 20 \\
13854 & B1 Iab & $1.50_{-0.5}^{+0.5}$ & 1.2 & 955 & 10 \\
190066 & B1 Iab & $0.70_{-0.1}^{+0.1}$ & 1.5 & 1275 & 15 \\
190603 & B1.5 Ia+ & $2.40_{-0.2}^{+0.0}$ & 1.2 & 390 & 15 \\
193183 & B1.5 Ib & $0.23_{-0.00}^{+0.27}$ & 1.5 & 565 & 20 \\
14818 & B2 Ia & $1.00_{-0.5}^{+0.5}$ & 1.5 & 625 & 15 \\
206165 & B2 Ib & $0.50_{-0.2}^{+0.0}$ & 1.5 & 640 & 15 \\
198478 & B2.5 Ia & $0.50_{-0.3}^{+0.0}$ & 1.2 & 550 & 20 \\
42087 & B2.5 Ib & $0.50_{-0.3}^{+0.0}$ & 1.2 & 650 & 15 \\
53138 & B3 Ia & $0.45_{-0.3}^{+0.0}$ & 1.2 & 500 & 20 \\
58350 & B5 Ia & $0.70_{-0.0}^{+0.3}$ & 1.0 & 320 & 20 \\
164353 & B5 Ib/II & $0.06_{-0.03}^{+0.0}$ & 1.5 & 450 & 25 \\
191243 & B5 Ib/II & $0.83_{-0.6}^{+0.0}$ & 1.0 & 550 & 20 \\
& & & & & \\
\hline
\end{tabular}

Fig. 11. Comparison of the relative strengths of the $\mathrm{N}$ and $\mathrm{C}$ lines in $\kappa$ Cas and HD 154090.

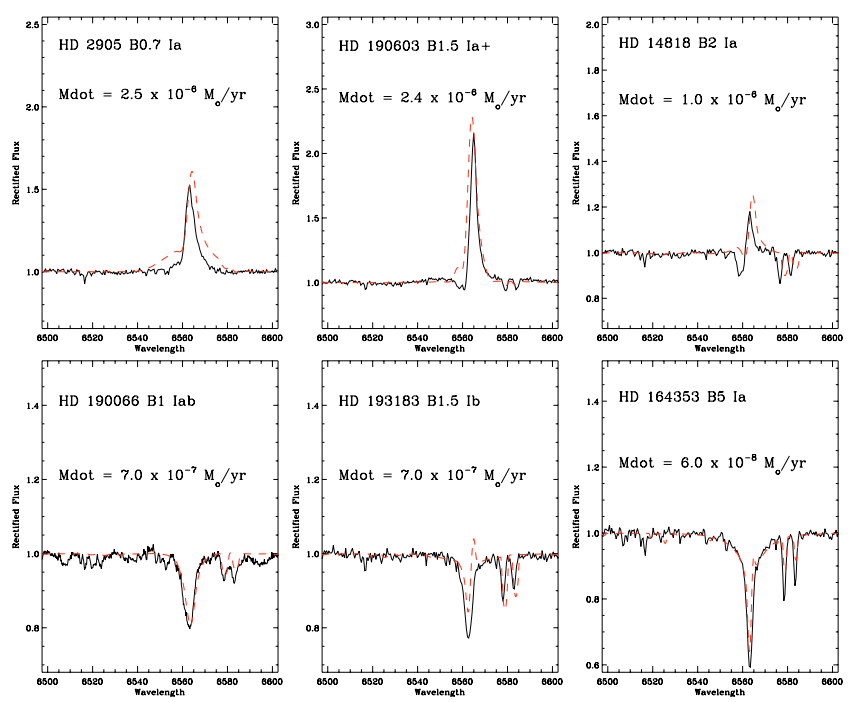

Fig. 12. Examples of CMFGEN fits to $\mathrm{H} \alpha$ profiles of $\kappa$ Cas, HD 190603 , HD 14818, HD 190066, HD 193183 and HD 164353, along with the mass loss rate adopted for each star. The red, dotted line represents the CMFGEN model fit to each $\mathrm{H} \alpha$ profile and the solid, black line indicates the observed $\mathrm{H} \alpha$ profile.

the same computation options are selected for both models to ensure that the density structure is computed using the same methods (e.g, the same number of $\Lambda$ iterations are specified). This is important as computational parameters may have been changed for individual models in order to ease convergence. For these reasons, a rigorous comparison of homogeneous and clumped models will be postponed to a later date.
These mass loss rates have been compared to those predicted by the theoretical mass loss prescription of Vink et al. (2000), as shown in Fig. 13, where the values of $T_{\text {eff }}, \log L_{*}$ and $M_{*}$ derived in the previous section have been input into the relevant mass loss recipes (Eqs. (12) and (13)) quoted in the paper. We find that the $\dot{M}$ 's derived here are in good agreement with the Vink et al. (2000) predictions, with discrepancies of a factor of 2, 3 on average and the maximum discrepancy a factor of 6 . Trundle et al. (2004); Trundle \& Lennon (2005) found that the values of $\dot{M}_{\text {vink }}$ were a factor of five lower than observed mass loss rates for early B supergiants, whereas for mid B supergiants $\dot{M}_{\text {vink }}$ was a factor of seven higher than observed values. No consistent discrepancy is found in our results but generally $\dot{M}_{\text {vink }} \leq \dot{M}_{\mathrm{H} \alpha}$ for B0-B1 supergiants and the reverse is true for B2-B3 supergiants. Our values of $\dot{M}$ obtained here were compared to those of Crowther et al. (2006), with who we have 8 stars in common, and the mass loss rates are in very good agreement. A comparison has also been made to the values obtained by Kudritzki et al. (1999), since there are again 8 stars common to both data sets (Fig. 14). With the exception of $\epsilon$ Ori, $\kappa$ Cas and HD 206165 , all the B supergiant mass loss rates derived by Kudritzki et al. (1999) are smaller than our values by typically a factor of up to 5. The values derived for $\epsilon$ Ori and $\kappa$ Cas are well within the errors of our derived values; however a larger discrepancy of a factor of $\sim 10$ is found for HD 206165. Initially this is puzzling since in both cases good fits have been obtained to the observed $\mathrm{H} \alpha$ profile of HD 206165 and do not suggest such a large discrepancy in $\dot{M}$. However, quite different stellar parameters have been adopted in terms of $T_{\text {eff }}\left(T_{\text {eff }}=18000 \mathrm{~K}\right.$ in our analysis cf. $20000 \mathrm{~K}$ from Kudritzki et al. 1999), $\log \left(L / L_{\odot}\right), R_{*}$ and $v_{\infty}$; more importantly Kudritzki et al. (1999) adopt a much higher $\beta$ value of 2.5 compared to 1.5 in this work. 


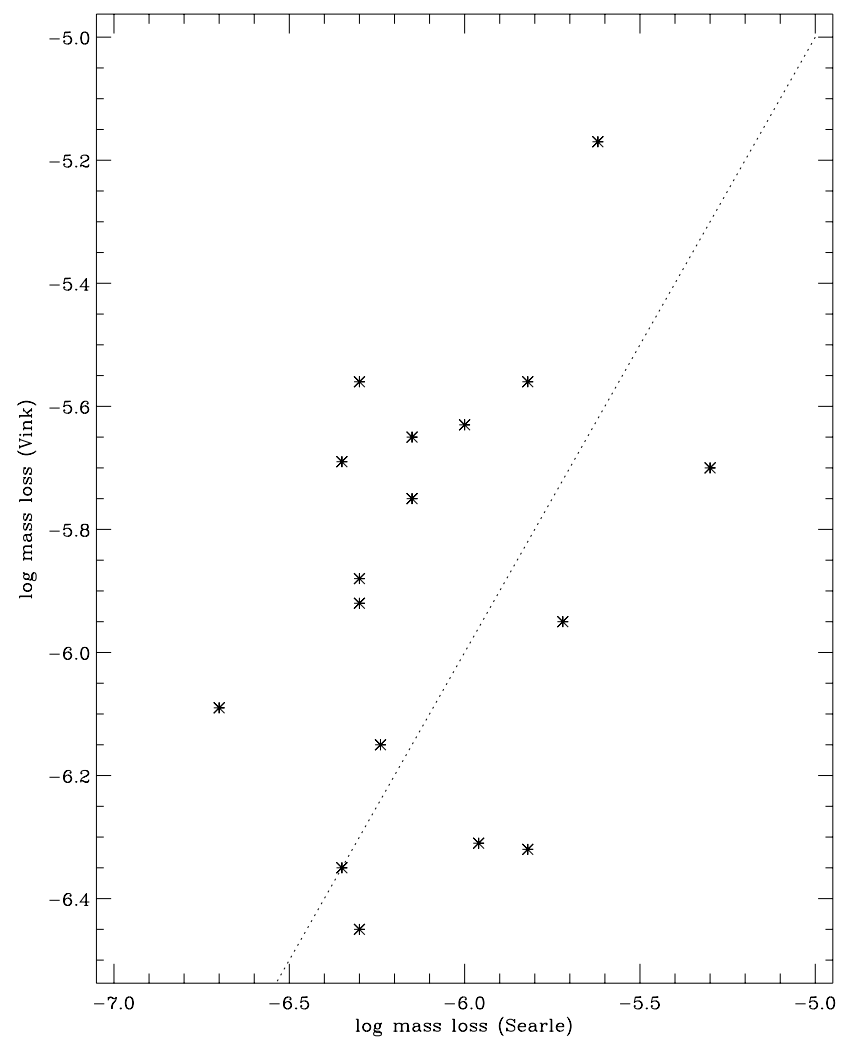

Fig. 13. Comparison of CMFGEN derived mass loss rates with theoretical mass loss rates predicted by the Vink et al. (2000) mass loss prescription. The dotted line indicates 1:1 correspondance.

\subsection{The wind-luminosity-momentum relation}

The concept of a wind-luminosity-momentum relation (hereafter WLR) was first proposed by Kudritzki et al. (1995), using the prediction from the theory of radiatively driven winds that there is a strong dependence of the total mechanical momentum flow $\dot{M} v_{\infty}$ of the stellar wind on stellar luminosity (e.g., Castor et al. 1975), which can be described as

$\dot{M} v_{\infty} \propto R_{*}^{-\frac{1}{2}} L^{\frac{1}{\alpha_{\mathrm{eff}}}}$.

The importance of the WLR lies in its potential as an extragalactic distance indicator provided that it is reliably calibrated. The proportionality shown in Eq. (4) was first confirmed observationally by Puls et al. (1996) for a sample of Galactic and Magellanic Cloud $\mathrm{O}$ stars with $5.5 \leq \log L_{*} \leq 6.5$. For $\log L_{*}<5.5$, a linear fit was not possible, demonstrating the dependence of the WLR on spectral type. Kudritzki et al. (1999) then showed that a linear fit to the WLR was also possible for galactic BA supergiants. Since then many authors (Repolust et al. 2004; Markova et al. 2004; Massey et al. 2004; Massey et al. 2005; Trundle et al. 2004; Trundle \& Lennon 2005) have published values for wind momenta when deriving fundamental parameters for sets of OBA stars using

$D_{\text {mom }}=\dot{M} v_{\infty} R_{*}^{0.5}$

where $R_{*}$ is in solar radii, $\dot{M}$ in $\mathrm{g} / \mathrm{s}$ and $v_{\infty}$ in $\mathrm{cm} / \mathrm{s}$ to give $D_{\text {mom }}$ in units of cgs. Assuming a WLR of the form

$\log D_{\text {mom }}=\log D_{0}+x \log \left(L / L_{\odot}\right)$

a linear regression can be used to constrain the coefficients $x$ and $\log D_{0}$. The reciprocal of $x$ can be thought of as the effective

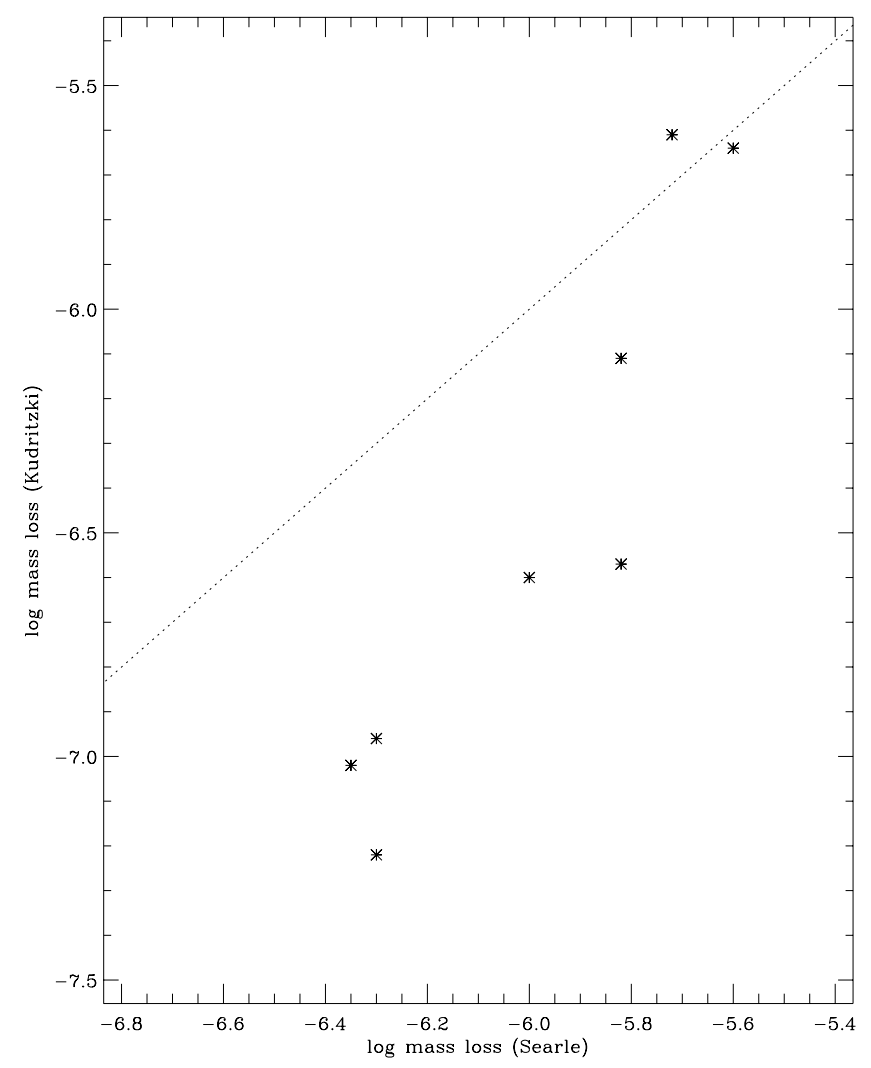

Fig. 14. Comparison of CMFGEN derived $\dot{M}$ with those of Kudritzki et al. (1999) for 8 stars common to both samples.

exponent $\alpha_{\mathrm{eff}}$ (see Eq. (4)). Applying a linear regression to our data gives $\log D_{0}=19.76$ and $x=1.61$. Looking at Fig. 15, it can be seen that the hotter spectral types i.e. O stars have a steeper WLR than cooler B spectral types. This is to be expected if, as proposed by Vink et al. (1999), Fe II and Fe III lines are responsible for driving the subsonic part of the wind, corresponding to lower ionisation stages. Figure 15 also illustrates the effect of metallicity on the WLR, with the more metal poor environment of the Magellanic Cloud hosting stars with lower values of $D_{\text {mom. }}$. This effect is particularly noticeable between Galactic and SMC B supergiants. Our values of $D_{\text {mom }}$ are compared to those predicted by the theoretical WLR prescription of Vink et al. (2000), using the parameters derived for our sample of stars in this chapter. It is found that the observational values are greater than predicted values for B0-B0.7 supergiants (except for the B0.2 Ia star HD 204172) where $T_{\text {eff }} \geq 23000 \mathrm{~K}$, which is expected for the hotter side of the bi-stability jump. Conversely, B1-B5 supergiants have smaller, observed values of $D_{\text {mom }}$ compared to predicted values. This is caused by a combination of several effects. Firstly, just as the predicted Vink et al. (2000) mass loss rates are a factor of 5 larger on the cooler side of the bi-stability jump, $\left(T_{\text {eff }} \sim 23000 \mathrm{~K}\right)$, the predicted wind momenta will be greater for B1-B5 supergiants, causing a larger discrepancy between observed and theoretical values of $D_{\text {mom }}$. In addition to this, many $\mathrm{B} 1-\mathrm{B} 5$ supergiants have $\mathrm{H} \alpha$ profiles in absorption, making it harder to constrain a "true" observed mass loss rate. Similar results are found by Trundle \& Lennon (2005) for their sample of SMC B supergiants. Repolust et al. (2004) suggested that the inclusion of clumping in the derivation of mass loss rates may help to alleviate the existing discrepancies between observed and theoretical wind momenta, which also exist for O stars as well as B stars. Clumping would reduce 


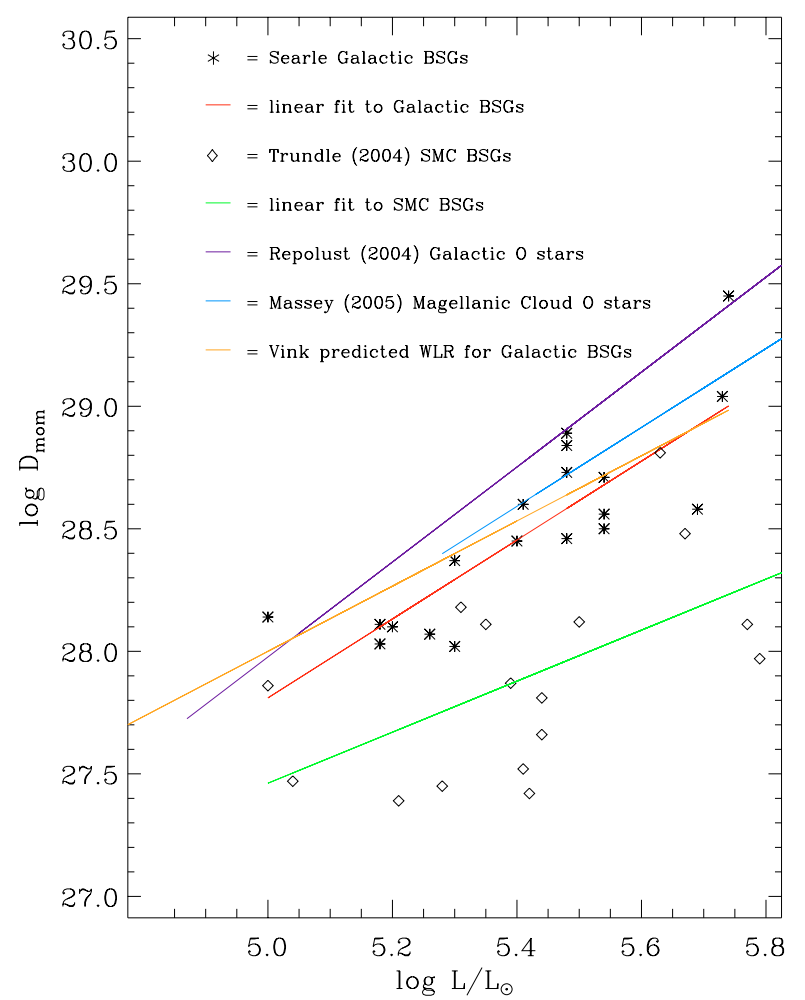

Fig. 15. The wind-luminosity momentum relation for OBA stars. Note the dependence of the WLR on spectral type and metallicity. The theoretical WLR predicted by Vink et al. (2000) is calculated for our sample of Galactic B supergiants and is represented by the orange line.

the mass loss rate and consequently lower wind momenta, although the precise amount of clumping present in OB star winds remains uncertain. Very recently Puls et al. (2006) attempted to derive better constraints on the clumping factor in hot star winds through a combined optical, infra-red and radio analysis of the wind. They found that use of clumped mass loss rates did produce much better agreement between observed and theoretical wind momenta, since for $\mathrm{O}$ stars the observed wind momenta were originally higher than the theoretical ones, but the inclusion of clumping reduced the value of $\dot{M}$ and consequently $D_{\text {mom }}$. Unfortunately, for the case of Galactic B supergiants this may only work for B0-B0.7 supergiants; the use of a lower, clumped $\dot{M}$ would not resolve the discrepancy for B1-B5 supergiants. This may indicate a fundamental difference between the structure and inhomogeneity of $\mathrm{O}$ and $\mathrm{B}$ star winds. At present, it is not possible to obtain a reliable calibration of the WLR, until the problems associated with its dependence on luminosity and metallicity, as well as the effect of clumping on observed mass loss rates, are resolved.

\section{Testing the UV predictions of CMFGEN}

The next step of our investigation was to examine if the CMFGEN models presented in the previous section would also provide a good fit to the UV silicon lines, thus confirming that $T_{\text {eff }}$ diagnostics at both optical and UV wavelengths implied the same value of $T_{\text {eff }}$ for each star. An example of a CMFGEN com-

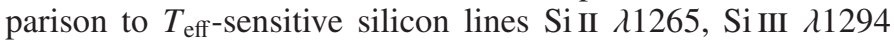
and $\lambda 1299$ (as noted by Massa 1989) is given in Fig. 16 for the B2 Ia star HD 14818 and the corresponding final CMFGEN model with $T_{\text {eff }}=18000 \mathrm{~K}, L=2.5 \times 10^{5} L / L_{\odot}$ and $\dot{M}=$ $1.1 \times 10^{-6} M_{\odot} \mathrm{yr}^{-1}$. Two other models with $T_{\text {eff }}=17500 \mathrm{~K}, L=$ $2.4 \times 10^{5} L / L_{\odot}, \dot{M}=1.2 \times 10^{-6} M_{\odot} \mathrm{yr}^{-1}$ and $T_{\mathrm{eff}}=18500 \mathrm{~K}$,
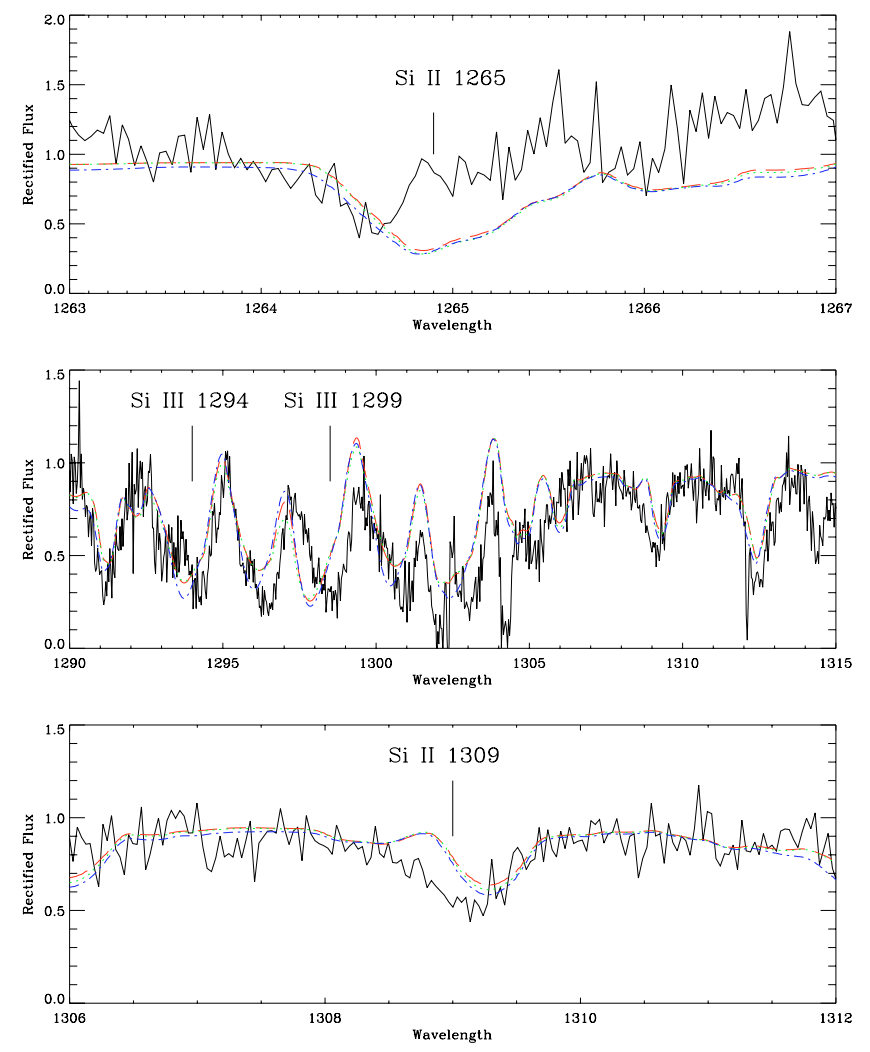

Fig. 16. CMFGEN model fit to the IUE spectrum of HD 14818 (B2 Ia), focusing on the UV silicon $T_{\text {eff }}$ diagnostics: Si II $\lambda 1265$ and $\lambda 1309$, Si III $\lambda 1294, \lambda 1299$ and $\lambda 1417$. The "best fit" model derived from the optical has $T_{\text {eff }}=18000 \mathrm{~K}, L=2.5 \times 10^{5} L / L_{\odot}$ and $\dot{M}=1.1 \times$ $10^{-6} M_{\odot} \mathrm{yr}^{-1}$ (dashed red line). Others models have $T_{\text {eff }}=17500 \mathrm{~K}$, $L=2.4 \times 10^{5} L / L_{\odot}, \dot{M}=1.2 \times 10^{-6} M_{\odot} \mathrm{yr}^{-1}$ (dotted green line) and $T_{\text {eff }}=18500 \mathrm{~K}, L=2.5 \times 10^{5} L / L_{\odot}, \dot{M}=1.8 \times 10^{-6} M_{\odot} \mathrm{yr}^{-1}$ (dotdashed blue line).

$L=2.5 \times 10^{5} L / L_{\odot}, \dot{M}=1.8 \times 10^{-6} M_{\odot} \mathrm{yr}^{-1}$ respectively are also shown to demonstrate the effects of changing $T_{\text {eff }}$ on these lines (the slight differences in the luminosity and mass loss of these models will not significantly affect the silicon lines). A direct comparison of the observed and model Si II $\lambda 1265$ line profiles is difficult since the continuum is raised about this line, but it is apparent that the model produces an asymmetric profile (whereas the observed profile is symmetric) shifted by about $1 \AA$ blue-ward relative to the observed line profile centre. The model profile is also much broader than the observed profile and varying $T_{\text {eff }}$ by $\pm 500 \mathrm{~K}$ has no significant effect on this line. In the case of Si II $\lambda 1309$, the model line profile is more narrow and shallow than the observed one. Changes in $T_{\text {eff }}$ are more apparent on this line, though still make no significant improvement to the overall line fit. For Si III $\lambda 1294$ and $\lambda 1299$, the model line profiles are again asymmetric, unlike the observed profiles, and the blue wings of these lines are overestimated whilst the absorption troughs are underestimated. In fact, both observed Si III lines appear to show some evidence of broadening due to the stellar wind despite being photospheric, which is also evident in the model profiles in the form of asymmetry. Additionally change in $T_{\text {eff }}$ appears to have no affect on these lines; however the higher value of $\dot{M}$ for the $T_{\text {eff }}=18500 \mathrm{~K}$ model (blue line) produces a deeper absorption trough for the profile. To conclude, varying $T_{\text {eff }}$ and even $\dot{M}$ has a small affect on these lines, but will not succeed in reproducing the observed lines accurately, with the correct broadness and symmetry. 


\subsection{The optical/UV discrepancy in wind lines}

A large number of UV lines are also strongly affected by mass loss from the wind, so it is also of interest to investigate whether the values of $\dot{M}$ derived from $\mathrm{H} \alpha$ in Sect. 4.6 succeed in reproducing the UV wind resonance lines accurately. This is not the first time that modelling of hot stars has been extended to the UV and matching the P Cygni profiles observed there. In the last couple of years, several authors have begun to consider both optical and UV stellar properties when deriving fundamental parameters (e.g., Evans et al. 2004a; Crowther et al. 2006) and Bouret et al. (2005) analysed IUE and FUSE spectra of two Galactic O4 stars with CMFGEN and TLUSTY, presenting one of the first analyses based exclusively on UV diagnostics that also uses these particular stellar atmosphere codes. It is evident that an optical analysis provides a much easier way of obtaining stellar parameters, where diagnostics for e.g. $T_{\text {eff }}$ and luminosity are readily available and only depend on abundance, $T_{\text {eff }}$ and/or luminosity. On the other hand, the task of identifying suitable diagnostic lines is less straightforward, since many UV lines will be sensitive to mass loss as well as $T_{\text {eff }}$, abundance and in some cases $v_{\text {turb }}$.

An example of a CMFGEN fit to the IUE spectrum of HD 190603 (B1.5 Ia+) is shown in Fig. 17. Since the mass loss rate has already been constrained from fits to the $\mathrm{H} \alpha$ profile, it is interesting to see whether the derived value of $\dot{M}$ is confirmed by reasonable fits to the UV P Cygni profiles, provided that a reasonable model fit to the $\mathrm{H} \alpha$ profile has already been achieved. Looking at the case of HD 190603 shown in Fig. 17, the fit to the observed $\mathrm{H} \alpha$ profile is good. However, it is clear that CMFGEN does not reproduce any of the observed P Cygni profiles accurately, implying that a different value of $\dot{M}$ would be appropriate for the UV. The model fails to produce sufficient high velocity absorption in the UV wind resonance lines, to the extent that the predicted CIV $\lambda \lambda 1548.2,1550.8$ line is only present as a photospheric line with no evidence of wind contamination. N V is not seen as a P Cygni profile in this star, but the model does not even produce a distinct, weak photospheric line at $1238 \AA$. However, better fits are achieved at lower ionisation: C II $\lambda \lambda$ 1335.66, 1335.71; Si IV $\lambda \lambda$ 1393.8, 1402.8 and Al III $\lambda \lambda$ 1854.7, 1862.8. The observed C II $\lambda \lambda$ 1335.66, 1335.71 line is saturated but the model produces an unsaturated line, which suggests that either a model with a higher value of $\dot{M}$ is required or the model ionisation is incorrect. Adopting a higher value for $\dot{M}$ though would worsen the effect of the model overestimating the red wings of the Si IV $\lambda \lambda 1393.8,1402.8$ doublet. It would have a more positive effect on the Al III $\lambda \lambda 1854.7,1862.8$ line, since the observed blueward doublet is beginning to saturate but the model blueward doublet is clearly unsaturated, again supporting a higher mass loss rate. The CMFGEN fit to $\mathrm{H} \alpha$ would worsen if a higher value of $\dot{M}$ was adopted, illustrating the discrepancy between the mass loss rates implied from the optical and UV. It is also noticeable when comparing the observed and model Si IV P Cygni profiles that the model doublet components are narrower than observed. As in the case of C IV, this is due to the model predicting to little absorption at high velocities. For HD 190603, these problems arise in spite of the fact that the value adopted for $\dot{M}$ provides a good fit to the $\mathrm{H} \alpha$ profile.

An example of a better CMFGEN fit to the UV wind resonance lines is given in Fig. 18 for the B2 Ia star HD 14818. The observed $\mathrm{H} \alpha$ profile displays a $\mathrm{P}$ Cygni profile, which has not been successfully reproduced by the model (as discussed in Sect. 4.6). Despite this, very good fits have been obtained to Si IV and Al III in comparison to those obtained for HD 190603, though
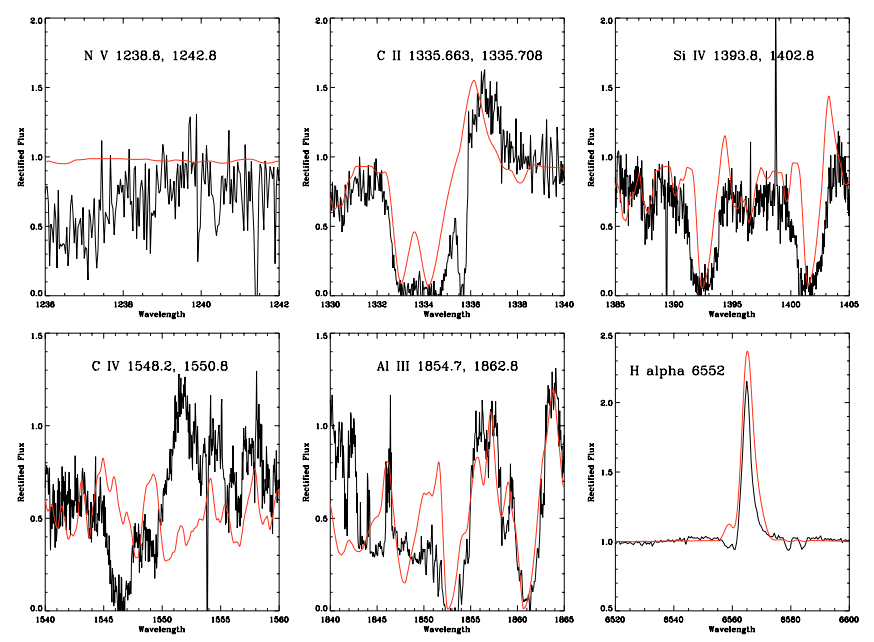

Fig. 17. CMFGEN fit to the IUE spectrum to the N v, C IV, Si IV, Al III and C II wind resonance lines of HD 190603 (B1.5 Ia+). Note that a good fit to $\mathrm{H} \alpha$ does not guarantee the same mass loss rate will provide a good fit to the UV P Cygni profiles. Model parameters are $T_{\text {eff }}=$ $19500 \mathrm{~K}, \log \left(L / L_{\odot}\right)=5.41$ and $\dot{M}=2.4 \times 10^{-6} M_{\odot}$.
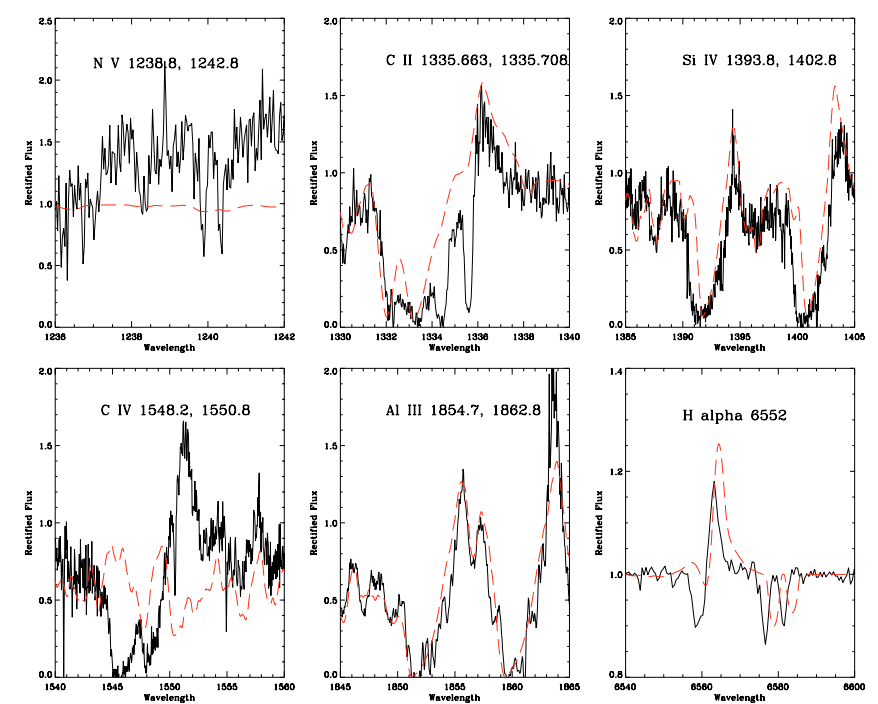

Fig. 18. CMFGEN fit to the IUE spectrum to the N v, C IV, Si IV, Al III and C II wind resonance lines of HD 14818 (B2 Ia). Even though the fit to $\mathrm{H} \alpha$ is not perfect, a reasonable fit is made to the UV P Cygni profiles, particularly Si IV and Al III. Model parameters are $T_{\text {eff }}=18000 \mathrm{~K}$, $\log \left(L / L_{\odot}\right)=5.40$ and $\dot{M}=1.0 \times 10^{-6} M_{\odot}$.

again a lack of high velocity absorption causes the model to under-estimate the broadness of the absorption trough for Si IV. However, the same failure occurs in reproducing the P Cygni profile of C IV line, whilst N V shows no evidence of wind contamination. The fit to $\mathrm{C}$ II is reasonable, although the model predicts too much redward emission and as a result does not match the redward side of the absorption trough. Conversely an example of a worse fit than either of the previous cases is shown in Fig. 19 for HD 53138 (B3 Ia). Its observed $\mathrm{H} \alpha$ profile is in absorption but shows a small amount of red-ward emission and is reasonably well matched by CMFGEN. On the other hand, the UV P Cygni profiles are in general poorly matched by the model, with none of the five wind line profiles being well reproduced. The same problems seen for HD 190603 and HD 14818 in matching N v, C IV and Si IV also occur here. The redward emission in C II is grossly over-estimated and the model 

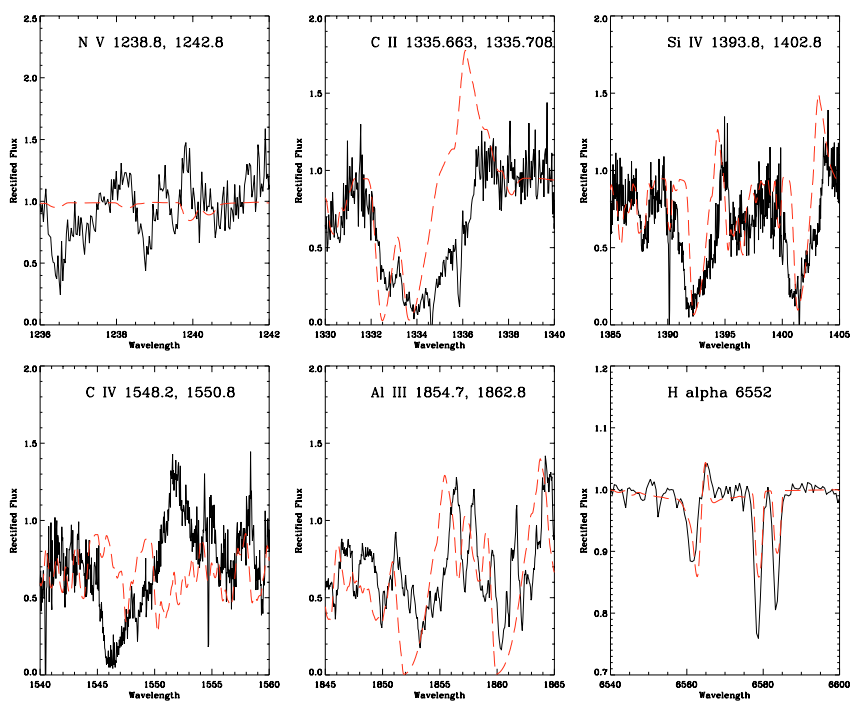

Fig. 19. CMFGEN fit to the IUE spectrum to the N v, C IV, Si IV, Al III and C II wind resonance lines of HD 53138 (B3 Ia). Although a good fit has been made to $\mathrm{H} \alpha$ with the adopted mass loss rate, CMFGEN does not reproduce the observed UV P Cygni profiles well. Model parameters are $T_{\text {eff }}=16500 \mathrm{~K}, \log \left(L / L_{\odot}\right)=5.30$ and $\dot{M}=4.5 \times 10^{-7} M_{\odot}$.

produces an asymmetric Al III profile that is not observed. In both cases the model predicts saturated lines when the observed profiles are not saturated (though $\mathrm{C}_{\mathrm{II}}$ is beginning to saturate a little). The high velocity absorption in Al III is over-estimated to the extent that it predicts saturation to occur at a higher velocity than observed. It is therefore clear from Figs. 17-19 that a discrepancy exists between the value of $\dot{M}$ required to fit the $\mathrm{H} \alpha$ and $\mathrm{UV}$ wind resonance lines (hereafter referred to as the optical/UV discrepancy).

In general, CMFGEN only succeeds in matching the C IV line when it is saturated in early B supergiants, at which point it is no longer sensitive to $T_{\text {eff }}$ and $\dot{M}$ so a reliable fit cannot be obtained as altering these parameters will have no affect on the model line profile. Otherwise, CMFGEN manages to reproduce most of the observed P Cygni profile for C II, Al III and Si IV, but fails to produce enough high velocity absorption to reproduce the full extent of the observed absorption trough. As a result, the model often under-estimates the blueward absorption as well as over-estimating the redward emission, especially in the case of Si IV. This can sometimes lead to the model giving an asymmetry to the P Cygni profile that is certainly not observed in the spectrum. Additionally, CMFGEN never succeeds in producing the $\mathrm{N}$ V P Cygni profile when present in $\mathrm{B} 0-\mathrm{B} 1 \mathrm{su}-$ pergiants and even when a weak, photospheric profile is observed, the model fails to produce a discernible spectral line at the correct wavelength for $\mathrm{N} \mathrm{V}$. In the hotter B supergiants, the model grossly underestimates the photospheric Al III and $\mathrm{C}$ II lines. However when the same resonance lines are seen as P Cygni profiles, the model has a tendency to reproduce them as saturated when they are observed to be unsaturated. All these discrepancies suggest that the problem lies within the predicted ionisation structure of the models. CMFGEN fits to the overall IUE spectra of 10 B0-B5 supergiants are available as online material (Figs. A.9-A.11). Very similar problems in matching the UV P Cygni profiles have also been encountered by Evans et al. (2004a) and Crowther et al. (2006) when modelling $\mathrm{O}$ and early B supergiants with CMFGEN.

\subsection{Modelling the UV exclusively}

The CMFGEN models examined in the last section demonstrate a clear discrepancy between $\dot{M}_{\mathrm{H} \alpha}$ and the value of $\dot{M}$ implied by the P Cygni profiles of the wind resonance lines. It is hardly surprising that they are unsuccessful in reproducing the observed UV wind diagnostics accurately. In this section, the possibility of modelling a star solely from its UV spectra will be investigated (ignoring any prior knowledge of values of parameters from the optical) to see if the UV can be reproduced more accurately. In order to do this, we must first identify suitable UV diagnostic lines by which values of $T_{\text {eff }}, \log \left(L / L_{\odot}\right), \dot{M}, v_{\infty}, \beta$ and abundances could be constrained.

Looking back to the problems mentioned in the previous section, one potential difficulty is immediately apparent. CMFGEN is unable to reproduce the CIV line accurately, which makes it hard to constrain $v_{\infty}$ and $\beta$ from this line. Suitable UV $T_{\text {eff }}$ diagnostics also need to be found besides the photospheric Si II and Si III lines discussed in Sect. 5. Si IV $\lambda \lambda$ 1393.8, 1402.8 could be a good candidate but it is also very sensitive to luminosity and mass loss; moreover it is often saturated, reducing its sensitivity to both parameters, and CMFGEN rarely reproduces it accurately. Other potential $T_{\text {eff }}$ diagnostics are Al III and C II which also show some sensitivity to mass loss and are therefore not ideal. Another possible $T_{\text {eff }}$ diagnostic is the photospheric Si II 1526.7, 1533.4 A line, but CMFGEN does not model these lines well either, often completely failing to reproduce the blueward part of the doublet. More importantly, the $1533.4 \AA$ doublet becomes blended with C IV $\lambda \lambda 1548.2,1550.8 \AA$ at high value of $v_{\infty}$. At this stage, we have no photospheric lines to use as reliable $T_{\text {eff }}$ diagnostics, since they are not well matched by CMFGEN. The best we can do is look at the UV lines best reproduced by CMFGEN (i.e., Si IV, Al III and C II) and analyse their sensitivity to the main stellar parameters.

In practice, another major problem materialises. It is difficult to disentangle the effects of $T_{\text {eff }}$ and $\dot{M}$ on Si IV, Al III and C II, plus they are often too saturated to be sensitive enough to these parameters. When Si IV is not observed to be saturated, CMFGEN still predicts a saturated profile that is virtually insensitive to $T_{\text {eff }}$ and $\dot{M}$, making it difficult to use as a $T_{\text {eff }}$ and $\dot{M}$ diagnostic. In fact, the lack of a significant difference between model P Cygni profiles when varying mass loss presents a serious obstacle to any attempt to derive parameters from the UV, as we will now show. For B0-B1 supergiants, the model often produces a saturated C IV P Cygni profile and over-estimates the Si IV P Cygni profile. It may appear logical that adopting a model with a lower mass loss rate would provide a better fit to the observed C IV and Si IV lines. However, the lack of sensitivity of this line to mass loss becomes apparent when the $\dot{M}$ adopted by the model is altered. This is illustrated in Fig. 20, where it can be seen that lowering the value of $\dot{M}$ from $5.0 \times 10^{-6}$ to $2.6 \times 10^{-6}$ has no affect on the wind resonance lines (implying that they are still optically thick), despite producing model $\mathrm{H} \alpha$ profiles in emission and absorption respectively (note that the broad feature seen in the model between $1242-1247 \AA$ is not $\mathrm{N}$ v but C III, which interestingly enough does show some sensitivity to mass loss). It could still be argued that a larger decrease in mass loss is required to fit these lines. However Fig. 21 disproves this idea as yet again no difference is seen between P Cygni profiles for models with $\dot{M}=$ $6 \times 10^{-8} M_{\odot} \mathrm{yr}^{-1}$ (red dashed line) and $=1.8 \times 10^{-7} M_{\odot} \mathrm{yr}^{-1}$ (blue dotted line) respectively. This is in spite of the fact that this difference in mass loss again results in model $\mathrm{H} \alpha$ profiles in emission and absorption, as well as having a significant difference on the amplitude of Ly $\alpha(1216 \AA)$. HD 164353 presents an 

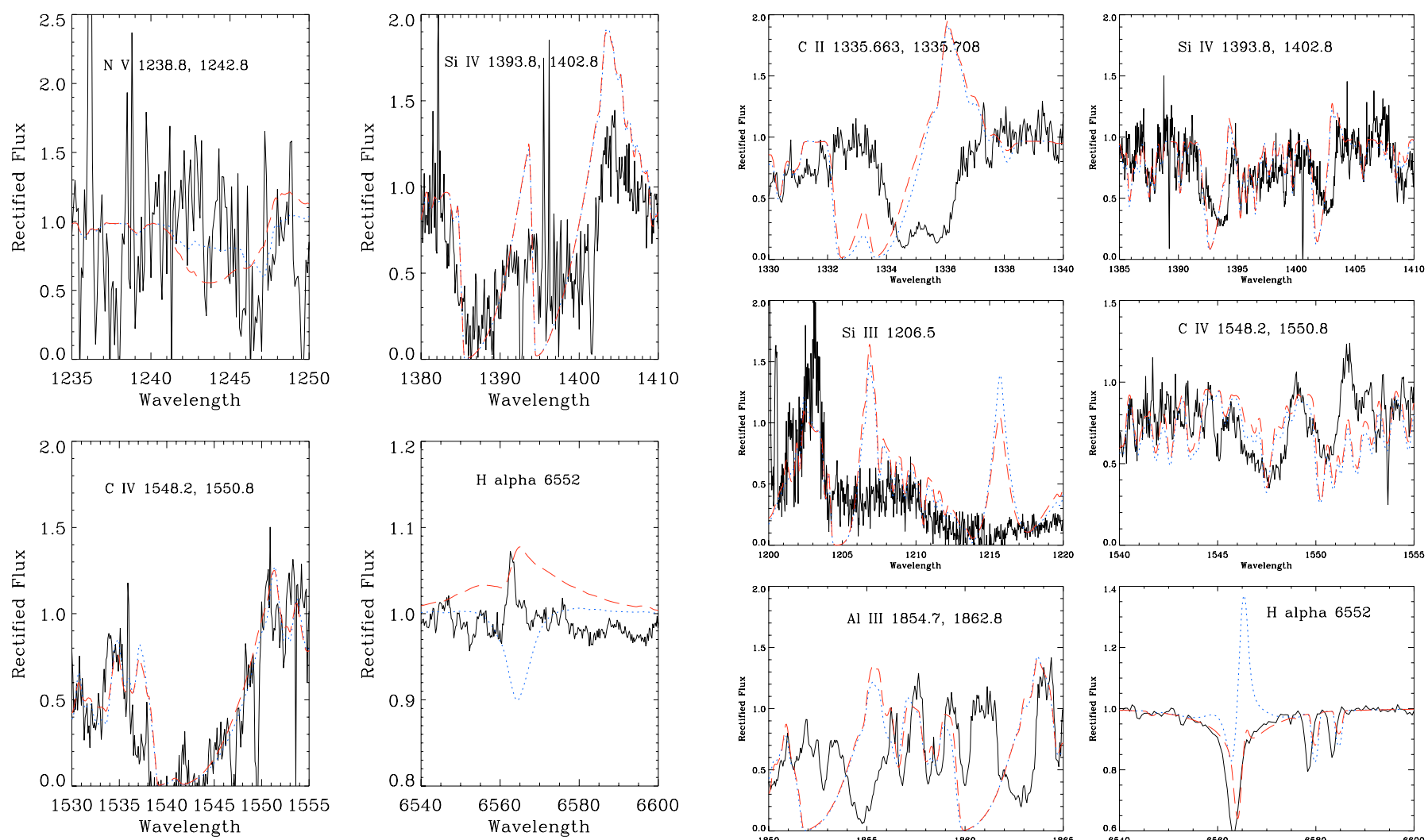

Fig. 20. Comparison of UV wind resonance lines of HD 192660 for models with $\dot{M}=5.0 \times 10^{-6} M_{\odot}$ (red dashed line) and $2.6 \times 10^{-6} M_{\odot}$ (blue dotted line) for HD 192660 (B0 Ib).

interesting case study for how CMFGEN deals with the ionisation in the stellar wind, as it is a B5 Ib/II star that possesses a very weak wind with $\dot{M}=6 \times 10^{-8} M_{\odot} \mathrm{yr}^{-1}$ and can be thought of as a star with negligible mass loss and stellar wind contamination. This is confirmed by looking at the observed C II and Al III resonance lines (Fig. 21), which show some asymmetric broadening. However the model predicts strongly saturated profiles for both lines despite the low mass loss rate adopted for the model, again suggesting that the predicted ionisation structure is at fault. This highlights another significant problem that, given their lack of sensitivity to significant changes in mass loss, the C IV and Si IV P Cygni profiles would not make suitable mass loss diagnostics. It appears that the root of the problem lies in CMFGEN predicting ionisation fractions for $\mathrm{C} \mathrm{II}$ and $\mathrm{Al}$ III that are too high, resulting in a large optical depth that produces too many absorbers at too high a velocity. The observed profiles on the other hand show us that absorption is only occurring around the rest velocity of the line. The model over-estimation of C II and Al III may therefore only be resolvable by lowering the ionisation fraction of these two elements and cannot be resolved by altering the mass loss rate of the model in question. From this, we conclude that the UV wind resonance lines are not suitable candidates for deriving $T_{\text {eff }}$ and $\dot{M}$. Even if the ionisation structure was correctly predicted, more diagnostic lines would be required to determine all the necessary stellar parameters other than $T_{\text {eff }}$ and $\dot{M}$, as well as ensuring that an accurate analysis had been carried out.

In addition to the wind resonance lines, the UV subordinate lines can potentially be used to provide additional constraints on the mass loss adopted for the model. An example is the Si IV 1122, $1128 \AA$ line in the FUV, whose upper energy level is coincident with the lower energy level of Si IV $1400 \AA$.
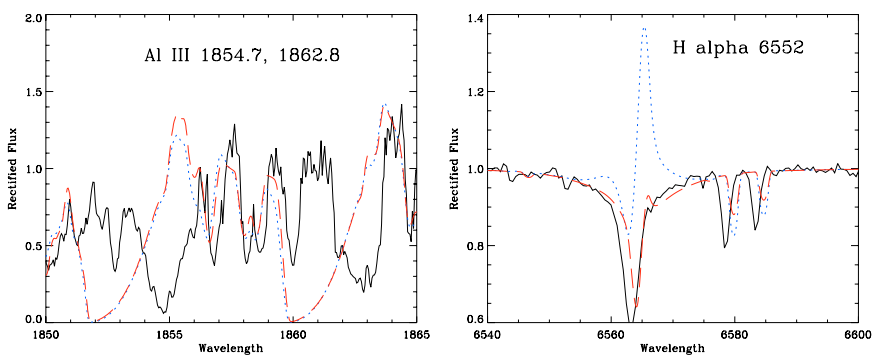

Fig. 21. Comparison of UV wind resonance lines of HD 164353 for models with $\dot{M}=6 \times 10^{-8} M_{\odot} \mathrm{yr}^{-1}$ (red dashed line) and $=1.8 \times$ $10^{-7} M_{\odot} \mathrm{yr}^{-1}$ (blue dotted line) respectively.

This means that if the model over-populates the lower level of Si IV $1400 \AA$, the upper level of Si IV 1122, $1128 \AA$ will also be over-populated, pushing the line into emission when it is observed to be in absorption. If this predicted line is seen to be in emission in a model when the observed line is in absorption, this is a direct indication that the adopted mass loss rate of the model is too high. There are no examples of Si IV $1128 \AA$ being in emission in the models used for the sample of 20 Galactic B supergiants, so this would suggest that the adopted mass loss rates are within reason. This also means that we could not have used this line as a mass loss diagnostic in this analysis.

It is possible to provide alternative perspectives on the UV behaviour by comparing the ionisation stages present in any given star, rather than trying to reproduce individual line profiles. Figure 22 shows the predicted ionisation structure against $w$ at four different $T_{\mathrm{eff}} ; 27500 \mathrm{~K}, 23500 \mathrm{~K}, 18000 \mathrm{~K}$ and $15000 \mathrm{~K}$ for the six ions (N V, C IV, Si IV, Si III, Al III and C II). CMFGEN predicts that Si IV will be dominant as expected for $T_{\text {eff }}=27500 \mathrm{~K}$, but shows very low levels of N V and C IV. This is hardly surprising since it explains the complete absence of a N v P Cygni profile (when present observationally), as well as the difficulties in generating a P Cygni profile for C IV when it is unsaturated. It is also interesting to note that the levels of ionisation drop off rapidly in the model as $w$ increases, contradicting the empirical determinations of Prinja et al. (2005) where winds became more highly ionised at high $w$. This is direct evidence of the model failing to generate enough high-velocity absorption to sustain the same level of ionisation further out in the wind. This is the reason for the "narrowness" of the model C IV and Si IV P Cygni profiles compared to the broad absorption troughs of the 

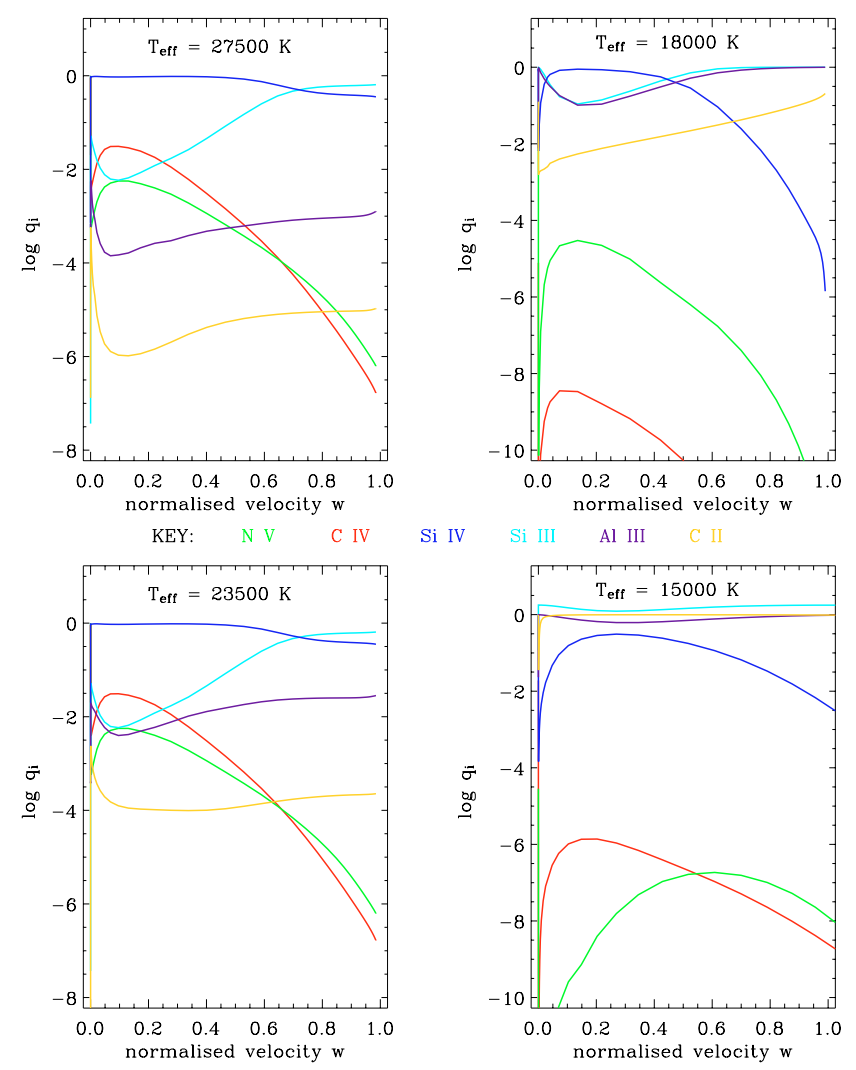

Fig. 22. CMFGEN predicted ionisation structure at different $T_{\text {eff }}$, plotted against normalised veocity $w$. The ions are colour-coded as follows: $\mathrm{NV}=$ green, $\mathrm{C}$ IV $=$ red, Si IV = dark blue, $\mathrm{Si}$ III = light blue, $\mathrm{Al} \mathrm{III}=$ purple and $\mathrm{C}$ II $=$ yellow. Si IV is predicted to be dominant for B0-B2 supergiants $\left(30000 \mathrm{~K} \leq T_{\text {eff }} \leq 18000 \mathrm{~K}\right)$, after which Si III, $\mathrm{Al}$ III and C II take over as the dominant ions in the wind for $T_{\text {eff }} \leq$ $18000 \mathrm{~K}$.

observed P Cygni profiles. If the model cannot sustain enough ionisation in the inner and outer parts of the wind, then it will be unable to fully reproduce the blue-ward part of the profile. CMFGEN predicts Si IV to be dominant down to $T_{\text {eff }}=18000 \mathrm{~K}$, at which point Si III and Al III take over as the dominant ions in the wind. At this $T_{\text {eff }}, \mathrm{C}$ II has also increased in strength, becoming a dominant ion at $T_{\text {eff }}=15000 \mathrm{~K}$. Whereas this approach has provided us with valuable insight into why CMFGEN struggles to predict the P Cygni profiles correctly, it too fails to provide us with an alternative means of constraining parameters due to the incorrectly-predicted ionisation structure.

Given all these problems with CMFGEN mismatching the observed UV P Cygni profiles, a investigation into the effects of clumping on these lines would not be worthwhile at present. In addition, mass loss in the UV is only sensitive to $\rho$, rather than $\rho^{2}$ as in $\mathrm{H} \alpha$ and radio-dominated regions of the wind, so it is not a particularly sensitive indicator of clumping. First the models need to predict the correction ionisation structure for B supergiants. Secondly, the problems associated with investigating the effects of clumping on $\mathrm{H} \alpha$ (as discussed in Sect. 4.6) need to be sorted as $\mathrm{H} \alpha$ is an important diagnostic of clumping and can provide important insight into its behaviour, which would aid a subsequent analysis of clumping in the UV. Furthermore, in comparison to $\mathrm{O}$ stars which possess strong indicators of UV clumping e.g., P V $\lambda \lambda$ 1118, 1128 (see Fullerton et al. 2006; Bouret et al. 2005), B supergiants do not possess an equally convenient UV diagnostic. A tentative examination of the effect of clumping on the UV profiles has shown that it does not improve the fits to the observed P Cygni profiles, as expected, but can alleviate the over-estimation of emission seen in the red-ward part of the model Si III and Al III profiles. In the case of the photospheric Si III lines around $\sim 1300 \AA$, some broadening of these lines due to the stellar wind is seen observationally, and the models also show some sensitivity to clumping in these lines. The models exaggerate the effect of the stellar wind on these lines by producing slightly asymmetric profiles, but the inclusion of clumping can help to lessen this asymmetry. This is logical since the inclusion of clumping will increase the wind density locally, providing more absorption at the point at which the line forms in the wind, helping to reduce the excess red-ward emission seen in many of the model P Cygni profiles. The inclusion of clumping will have no affect on saturated lines in the model as they are no longer sensitive to density changes in the wind. All the aforementioned issues associated with investigating clumping effects need to be addressed before any truly meaningful analysis of clumping in the UV can be carried out.

\section{Conclusions}

A quantitative study of the optical and UV properties of B0-B5 Ia, Iab, Ib/II supergiants has been carried out, using the nLTE, line-blanketed stellar atmosphere code of Hillier \& Miller (1998). A revised B supergiant $T_{\text {eff }}$ scale (derived using a stellar atmosphere code that includes the effects of line blanketing) has been presented, giving a range of $14.5000 \mathrm{~K} \leq T_{\text {eff }} \leq 30000 \mathrm{~K}$ for these stars. This scale shows a drop of up to $10000 \mathrm{~K}$ from $\mathrm{B} 0 \mathrm{Ia} / \mathrm{b}$ to B1 Iab and a difference of up to $2500 \mathrm{~K}$ between Ia and Ib stars. It also shows that on average the effect of including line blanketing in the model produces a modest reduction of up to $1000 \mathrm{~K}$ for B0-B0.7 and B3-B5 supergiants, whereas a larger reduction of up to $3000 \mathrm{~K}$ is seen for $\mathrm{B} 1-\mathrm{B} 2$ supergiants (see Table 4). The 20 Galactic B supergiants also displayed a range of $2.1 \leq \log g \leq 3.4$ in surface gravity. These results, together with those of Crowther et al. (2006), have been used to construct a new set of averaged fundamental parameters for B0-B5 supergiants, according to spectral type. Mass loss rates derived from $\mathrm{H} \alpha$ proved B supergiant winds to be generally weaker than those of $\mathrm{O}$ supergiants (as expected since they are lower-luminosity objects) with $\dot{M}$ ranging from $-7.22 \leq \log \dot{M} \leq-5.30$. All 20 B supergiants also shown signs of CNO processing, with the largest nitrogen enrichments being seen for B1-B2 supergiants. Evidence for a mass discrepancy is found between estimates of $M_{\text {spec }}$ and $M_{\text {evol }}$, with the largest differences peaking at a value of $\log \left(L / L_{\odot}\right) \sim 5.4$.

A wind-momentum-luminosity relation has also been derived for our sample, which is lower in value for B1-B5 supergiants than that predicted by Vink et al. (2000), but greater than predicted values for $\mathrm{B} 0-\mathrm{B} 0.7$ supergiants. For this reason it is not possible to reconcile this difference in observed and theoretical WLRs over the whole B supergiant spectral range by adopting clumped $\dot{M}$ as is the case for O stars. A severe problem exists in the form of the optical-UV discrepancy, where the model fails to reproduce some of the P Cygni profiles accurately. This highlights a failure in the model to generate enough high-velocity absorption to succeed in reproducing the observed P Cygni profile and more crucially highlights that the models are not predicting the correct ionisation structure. Given that B supergiants, along with other massive stars, have their peak flux in the UV, it is imperative that this discrepancy is resolved if we are to have confidence that fundamental parameters derived by this method are a true representation of the star's properties. Furthermore it underlines the incompleteness of our current understanding of 
the physics of massive star winds and the necessity to review the standard model. A more thorough analysis of the ionisation structure of early B supergiant winds will be presented in Paper II.

Acknowledgements. S.C.S. would like to acknowledge PPARC for financial support. Thanks go to John Hillier for assistance in using CMFGEN as well as Callum Wright and Jeremy Yates for computing support.

\section{References}

Anderson, L. S. 1989, ApJ, 339, 588

Bianchi, L., \& Garcia, M. 2002, ApJ, 581, 610

Bidelman, W. P. 1988, PASP, 100, 1084

Blomme, R., Prinja, R. K., Runacres, M. C., \& Colley, S. 2002, A\&A, 382, 921

Bouret, J.-C., Lanz, T., Hillier, D. J., et al. 2003, ApJ, 595, 1182

Bouret, J.-C., Lanz, T., \& Hillier, D. J. 2005, A\&A, 438, 301

Brown, A. G. A., de Geus, E. J., \& de Zeeuw, P. T. 1994, A\&A, 289, 101

Castor, J. I., Abbott, D. C., \& Klein, R. I. 1975, ApJ, 195, 157

Cranmer, S. R., \& Owocki, S. P. 1996, ApJ, 462, 469

Crowther, P. A., Hillier, D. J., Evans, C. J., et al. 2002, ApJ, 579, 774

Crowther, P. A., Lennon, D. J., \& Walborn, N. R. 2006, A\&A, 446, 279

de Zeeuw, P. T., Hoogerwerf, R., de Bruijne, J. H. J., Brown, A. G. A., \& Blaauw, A. 1999, AJ, 117,354

Dufton, P. L., Ryans, R. S. I., Trundle, C., et al. 2005, A\&A, 434, 1125

Egret, D. 1978, A\&A, 66, 275

Evans, C. J., Crowther, P. A., Fullerton, A. W., \& Hillier, D. J. 2004a, ApJ, 610, 1021

Evans, C. J., Lennon, D. J., Trundle, C., Heap, S. R., \& Lindler, D. J. 2004b, ApJ, 607, 451

Fernie, J. D. 1983, ApJS, 52, 7

Fitzgerald, M. P. 1970, A\&A, 4, 234

Fullerton, A. W., Massa, D. L., \& Prinja, R. K. 2006, ApJ, 637, 1025

Fullerton, A. W., Massa, D. L., Prinja, R. K., Owocki, S. P., \& Cranmer, S. R. 1997, A\&A, 327, 699

Garmany, C. D., \& Stencel, R. E. 1992, A\&AS, 94, 211

Herrero, A., Puls, J., \& Najarro, F. 2002, A\&A, 396, 949

Hillier, D. J., \& Miller, D. L. 1998, ApJ, 496, 407

Hillier, D. J., \& Miller, D. L. 1999, ApJ, 519, 354

Hillier, D. J., Lanz, T., Heap, S. R., et al. 2003, ApJ, 588, 1039

Hiltner, W. A. 1956, ApJS, 2, 389

Hoffleit, D., \& Jaschek, C. 1982, The Bright Star Catalogue (New Haven: Yale University Observatory), 4th edn.
Howarth, I. D., Siebert, K. W., Hussain, G. A. J., \& Prinja, R. K. 1997, MNRAS, 284,265

Hubeny, I., \& Lanz, T. 1995, ApJ, 439, 875

Humphreys, R. M. 1978, ApJS, 38, 309

Kaufer, A., Stahl, O., Prinja, R. K., \& Witherick, D. 2006, A\&A, 447, 325

Kaufer, A., Prinja, R. K., \& Stahl, O. 2002, A\&A, 382, 1032

Kudritzki, R.-P., Lennon, D. J., \& Puls, J. 1995, in Science with the VLT, ed. J. R. Walsh, \& I. J. Danziger, 246

Kudritzki, R.-P., \& Puls, J. 2000, ARA\&A, 38, 613

Kudritzki, R. P., Puls, J., Lennon, D. J., et al. 1999, A\&A, 350, 970

Lanz, T., \& Hubeny, I. 2003, ApJS, 146, 417

Lennon, D. J., Dufton, P. L., \& Fitzsimmons, A. 1992, A\&AS, 94, 569

Lesh, J. R. 1968, ApJS, 17, 371

Maeder, A., \& Meynet, G. 2001, A\&A, 373, 555

Markova, N., Puls, J., Repolust, T., \& Markov, H. 2004, A\&A, 413, 693

Martins, F., Schaerer, D., \& Hillier, D. J. 2005, A\&A, 436, 1049

Massa, D. 1989, A\&A, 224, 131

Massa, D., Prinja, R. K., \& Fullerton, A. W. 1995, ApJ, 452, 842

Massa, D., Fullerton, A. W., Sonneborn, G., \& Hutchings, J. B. 2003, ApJ, 586, 996

Massey, P., Bresolin, F., Kudritzki, R. P., Puls, J., \& Pauldrach, A. W. A. 2004, ApJ, 608, 1001

Massey, P., Puls, J., Pauldrach, A. W. A., et al. 2005, ApJ, 627, 477

McErLean, N. D., Lennon, D., \& Dufton, P. 1999, A\&A, 349, 553

Meynet, G., \& Maeder, A. 2000, A\&A, 361, 101

Morel, T., Marchenko, S. V., Pati, A. K., et al. 2004, MNRAS, 351, 552

Nieva, M. F., \& Przybilla, N. 2006, ApJ, 639, L39

Owocki, S. P., Castor, J. I., \& Rybicki, G. B. 1988, ApJ, 335, 914

Prinja, R. K., Rivinius, T., Stahl, O., et al. 2004, A\&A, 418, 727

Prinja, R. K., Massa, D., \& Fullerton, A. W. 2002, A\&A, 388, 587

Prinja, R. K., Massa, D., \& Searle, S. C. 2005, A\&A, 430, L41

Puls, J., Kudritzki, R.-P., Herrero, A., et al. 1996, A\&A, 305, 171

Puls, J., Markova, N., Scuderi, S., et al. 2006, A\&A, submitted

Repolust, T., Puls, J., \& Herrero, A. 2004, A\&A, 415, 349

Runacres, M. C., \& Owocki, S. P. 2002, A\&A, 381, 1015

Rusconi, L., Sedmak, G., Stalio, R., \& Arpigny, C. 1980, A\&AS, 42, 347

Schild, H. 1985, A\&A, 146, 113

Townsend, R. H. D. 1997, MNRAS, 284, 839

Trundle, C., \& Lennon, D. J. 2005, A\&A, 434, 677

Trundle, C., Lennon, D. J., Puls, J., \& Dufton, P. L. 2004, A\&A, 417, 217

Venn, K. A. 1995, ApJ, 449, 839

Venn, K. A. 1999, ApJ, 518, 405

Vink, J. S., de Koter, A., \& Lamers, H. J. G. L. M. 1999, A\&A, 350, 181

Vink, J. S., de Koter, A., \& Lamers, H. J. G. L. M. 2000, A\&A, 362, 295

Walborn, N. R. 1971, ApJS, 23, 257

Walborn, N. R. 1972, AJ, 77, 312

Walborn, N. R. 1976, ApJ, 205, 419 


\section{Appendix A: Error analysis}

In this section, the errors affecting each derived parameter are discussed. The error on $T_{\text {eff }}$ is estimated from the quality of the CMFGEN model fit to the diagnostic silicon, helium and magnesium lines and therefore represents the range in $T_{\text {eff }}$ over which a satisfactory fit to the observed spectrum of the star could be obtained. Luminosity is primarily constrained through dereddening the observed spectra with respect to the model spectral distribution, its error depends on $\Delta M_{V}$, whose errors are estimated from dereddening the observed spectrum with respect to the model spectral energy distribution. $\Delta \log L_{*}$ also depends on $\Delta M_{\mathrm{bol}}$, since $M_{\mathrm{bol}}=M_{V}+B . C$., therefore $\Delta \log L_{*}$ is calculated as

$\Delta \log L_{*}=\log L_{*} \frac{2.5 \Delta M_{\mathrm{bol}}}{\left(M_{\mathrm{bol}}-4.72\right)}$.

The error on $R_{*}$ depends on the square root of the sum of $\left(\Delta T_{\mathrm{eff}}\right)^{2}$ and $\left(\Delta L_{*}\right)^{2}$, with $\Delta L_{*}$ having the greatest influence on $\Delta R_{*}$. For 15 out of the $20 \mathrm{~B}$ supergiants, $\Delta R_{*}$ is within $10 \%$ of the absolute value of $R_{*}$; those stars with larger $\Delta R_{*}$ are discussed separately in this section. The error on $\log g$ for 16 of the 20 stars star is estimated to be 0.25 dex, based on the accuracy of line fits and the effect of $\Delta T_{\text {eff }}$ in determining $\log g$. For four of the stars in our sample, we adopted $\Delta \log g=0.38$ dex. In the cases of HD 190603 and HD 14818, this was due to asymmetric nature of the $\mathrm{H} \gamma$ and $\mathrm{H} \delta$ profiles, whereas for HD 64760 and HD 13854 it reflected the larger error in $T_{\text {eff }}$ of $2000 \mathrm{~K}$ (cf. to $500-1500 \mathrm{~K}$ for other stars in the sample. The resulting uncertainty on the spectroscopic mass, $M_{\text {spec }}$, due to errors in constraining $\log g$ and $R_{*}$ range from $0.08 \leq \Delta M_{*} \leq 14.52$. In comparison, $\Delta M_{\text {evol }}$ is typically $5 M_{\odot}$ as shown in Fig. 8, which demonstrates the effect of assuming different values of $T_{\text {eff }}$ and $\log \left(L / L_{\odot}\right)$ on a star's position in the HR diagram.

Determining the error in constraining the mass loss rate is more complicated, since it depends on both $\Delta R_{*}$ and the error in fitting the $\mathrm{H} \alpha$ profile by varying $\dot{M}$ and $\beta$. However, the errors incurred from uncertainties in deriving $R_{*}$ are negligible compared to those arising from fitting the $\mathrm{H} \alpha$ profile, so we are justified in defining $\Delta \dot{M}$ as solely the error in fitting the $\mathrm{H} \alpha$ profile, accounting for the degeneracy in varying $\beta$ to fit $\mathrm{H} \alpha$ profiles in emission. Values of $v_{\infty}$ are taken from SEI analysis of UV wind resonance lines (the result of which will be presented in Paper II) and are accurate to $\pm 50 \mathrm{~km} \mathrm{~s}^{-1}$. The values for $v_{\text {turb }}$ are constrained with an uncertainty of $\pm 5 \mathrm{~km} \mathrm{~s}^{-1}$, as dictated by sensitivity of fitting the Si III lines by eye.

Some uncertainties exist in our analysis that warrant further discussion. Although for the majority of stars it was possible to constrain $T_{\text {eff }}$ within $\pm 1000 \mathrm{~K}$, this was not possible for the stars HD 64760 and HD 13854. HD 64760 is a rapid rotator and the large width of its spectral lines makes it harder to make an accurate distinction between model fits whose $T_{\text {eff }}$ differ by $1000 \mathrm{~K}$, resulting in $\Delta T_{\text {eff }}= \pm 2000 \mathrm{~K}$ for this star. In the case of HD 13854, if the adopted $T_{\text {eff }}$ of $20000 \mathrm{~K}$ is increased to $22000 \mathrm{~K}$ (keeping the same luminosity) then a much better fit is made to the silicon lines (i.e., Si IV 4089 and Si III 4552, 4568 and 4575) but at the expense of grossly over-estimating the hydrogen and helium lines (i.e., $\mathrm{H} \beta, \mathrm{H} \gamma, \mathrm{H} \delta$, $\mathrm{He}$ I 4121, 4144, 4387 and 4471). Normally in our analysis, the fitting of silicon lines would be given priority, but given the weakness of Si IV 4089 at this spectral type (B1 Iab), it is reasonable to assign greater importance to fitting the helium lines. Furthermore, adopting a model that only fits the silicon lines well and largely overestimates the hydrogen and helium lines will give a misleading indication of the value of $T_{\text {eff }}$. HD 13854 also has quite a large error in $M_{V}$, which consequently propagates into significant uncertainties in $\log \left(L / L_{\odot}\right)$ and, combined with a larger $\Delta T_{\text {eff }}$, leads to a very large $\Delta R_{*}$. This arises from a noisy IUE SWP spectrum and the absence of a LWR spectrum leading to a large dereddening error; the same is true for the considerable errors on the values of $M_{V}$ and $R_{*}$ obtained for HD 204172. Low quality IUE spectra generating higher $\Delta E(B-V)$ also explain the $\Delta \log \left(L / L_{\odot}\right)$ found for HD 14818 and HD 206165. However, for the B5 II (Ib) star HD 164353, it is the value of $M_{V}=-4.2$ that poses a problem; CMFGEN simply fails to calculate a succesfully-converged model at the required luminosity. This explains the large values of $\Delta \log \left(L / L_{\odot}\right), \Delta R_{*}$ and $\Delta M_{V}$. The adopted value of $M_{V}$ has been independently confirmed by several different sources in the literature so we believe it to be correct. Furthermore, four stars (HD 37128, HD 192660, HD 198478 and HD 42087) have larger errors in the observed value of $M_{V}$ than the value of $M_{V}$ derived from dereddening, so in practise the quoted value of $\Delta M_{V}$ could be up to $0.2 \mathrm{mag}$ larger. 
S. C. Searle et al.: Fundamental parameters of Galactic B supergiants. I., Online Material p 2

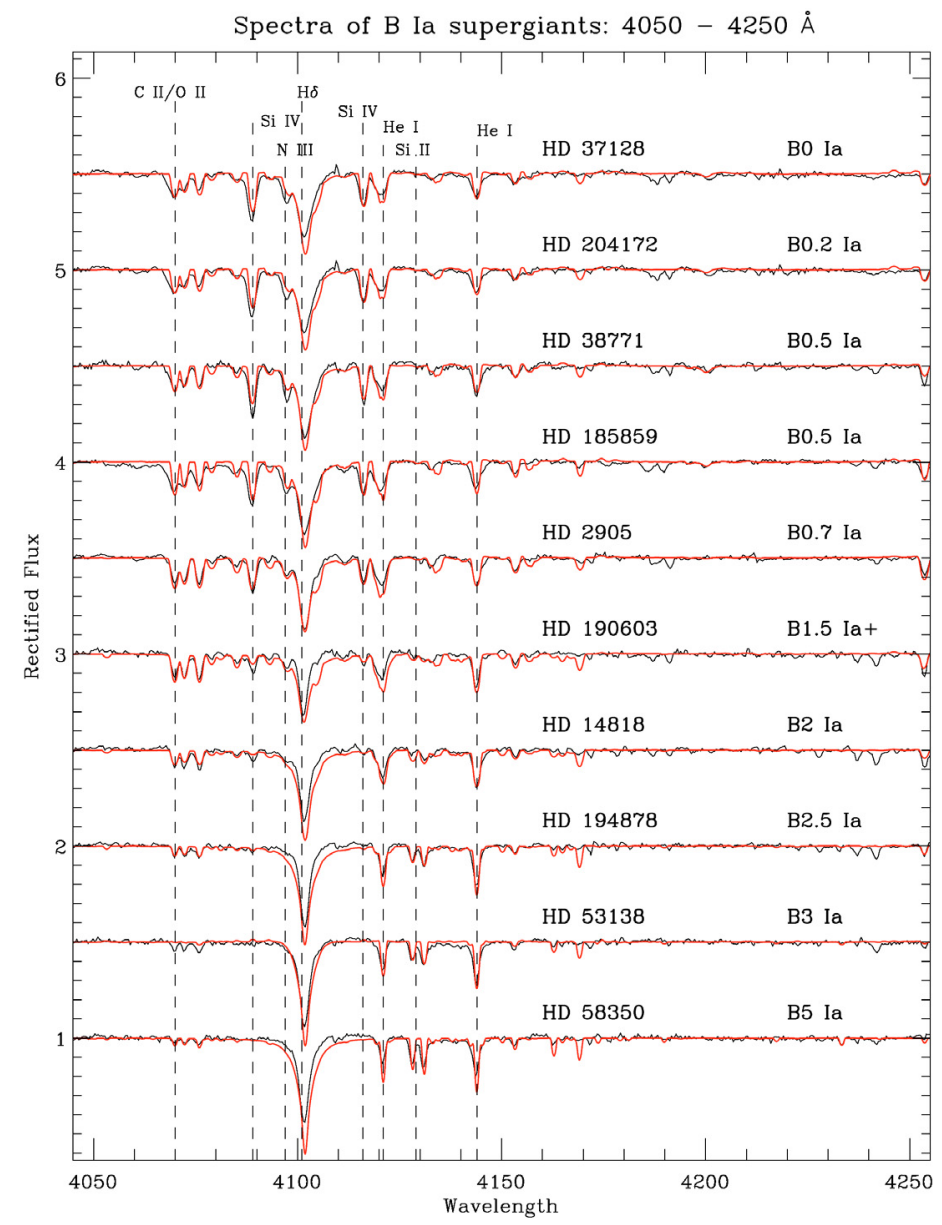

Fig. A.1. CMFGEN model fits (4050-4250 ̊) to the optical spectra of $10 \mathrm{~B}$ Ia supergiants, with the $T_{\text {eff }}$, luminosity and CNO diagnostic lines marked as shown. Optical spectrum is in black, CMFGEN model fit is shown in red. 
S. C. Searle et al.: Fundamental parameters of Galactic B supergiants. I., Online Material p 3

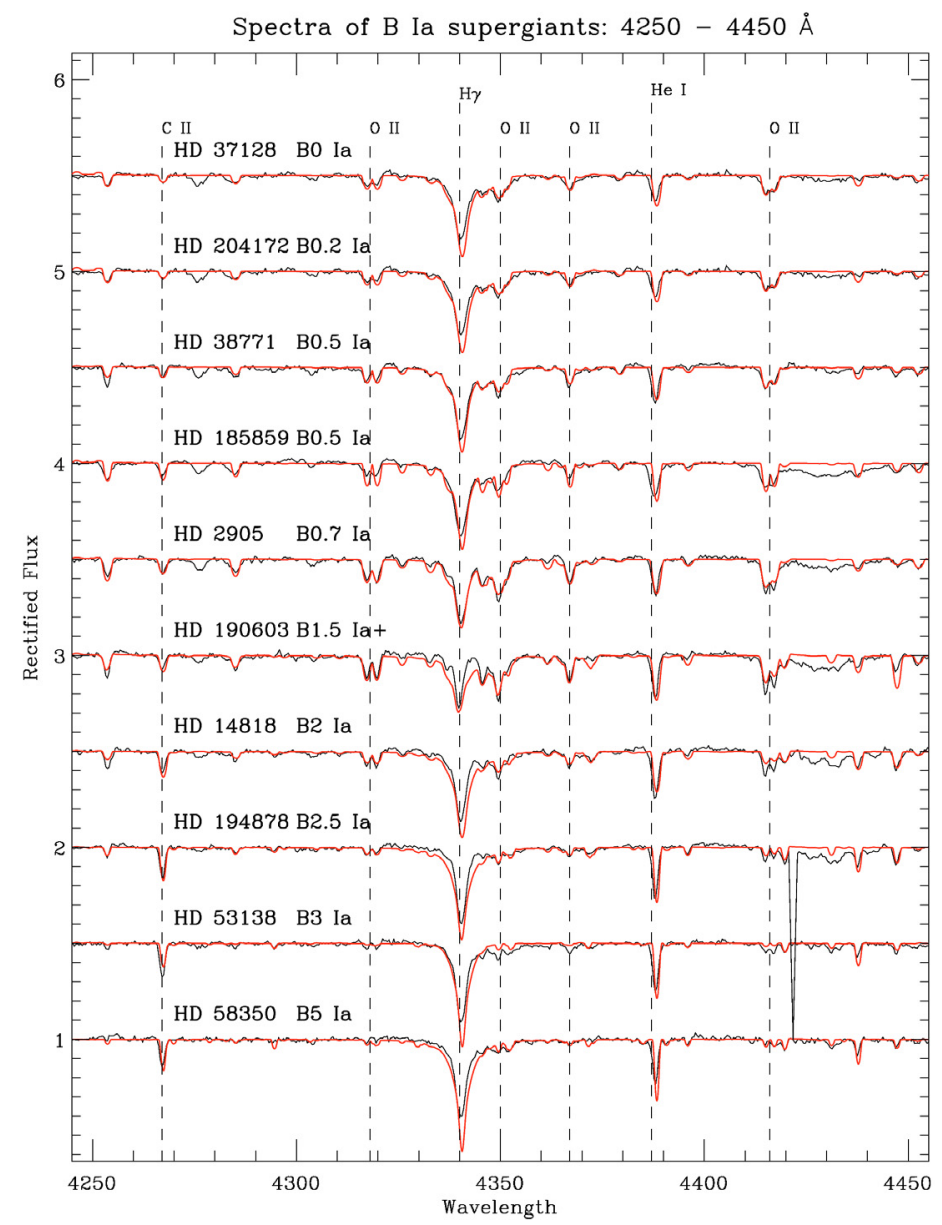

Fig. A.2. CMFGEN model fits (4250-4450 $\AA$ ) to the optical spectra of $10 \mathrm{~B}$ Ia supergiants, with the $T_{\text {eff }}$, luminosity and CNO diagnostic lines marked as shown. Optical spectrum is in black, CMFGEN model fit is shown in red. 
S. C. Searle et al.: Fundamental parameters of Galactic B supergiants. I., Online Material p 4

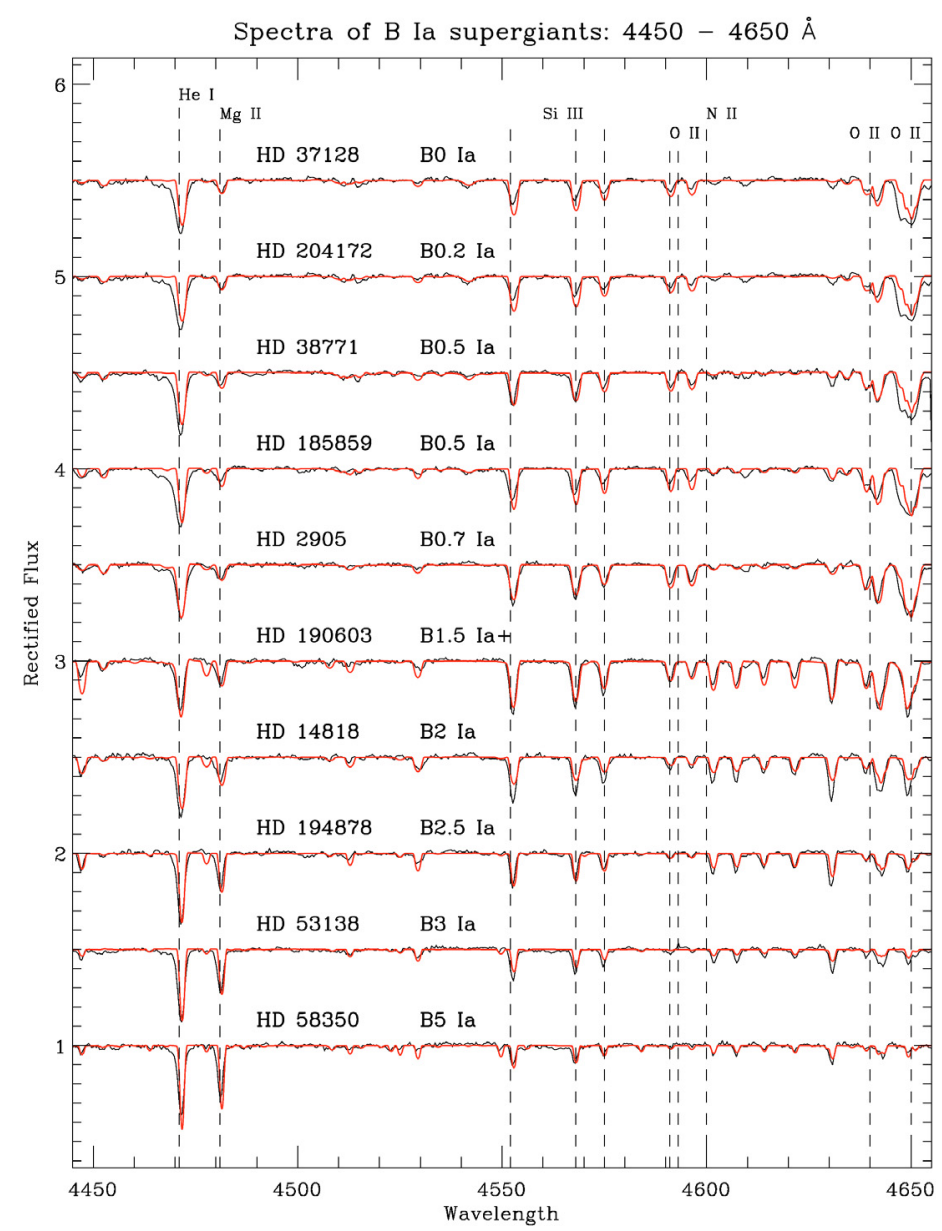

Fig. A.3. CMFGEN model fits (4450-4650 $\AA$ ) to the optical spectra of 10 B Ia supergiants, with the $T_{\text {eff }}$, luminosity and CNO diagnostic lines marked as shown. Optical spectrum is in black, CMFGEN model fit is shown in red. 
S. C. Searle et al.: Fundamental parameters of Galactic B supergiants. I., Online Material p 5

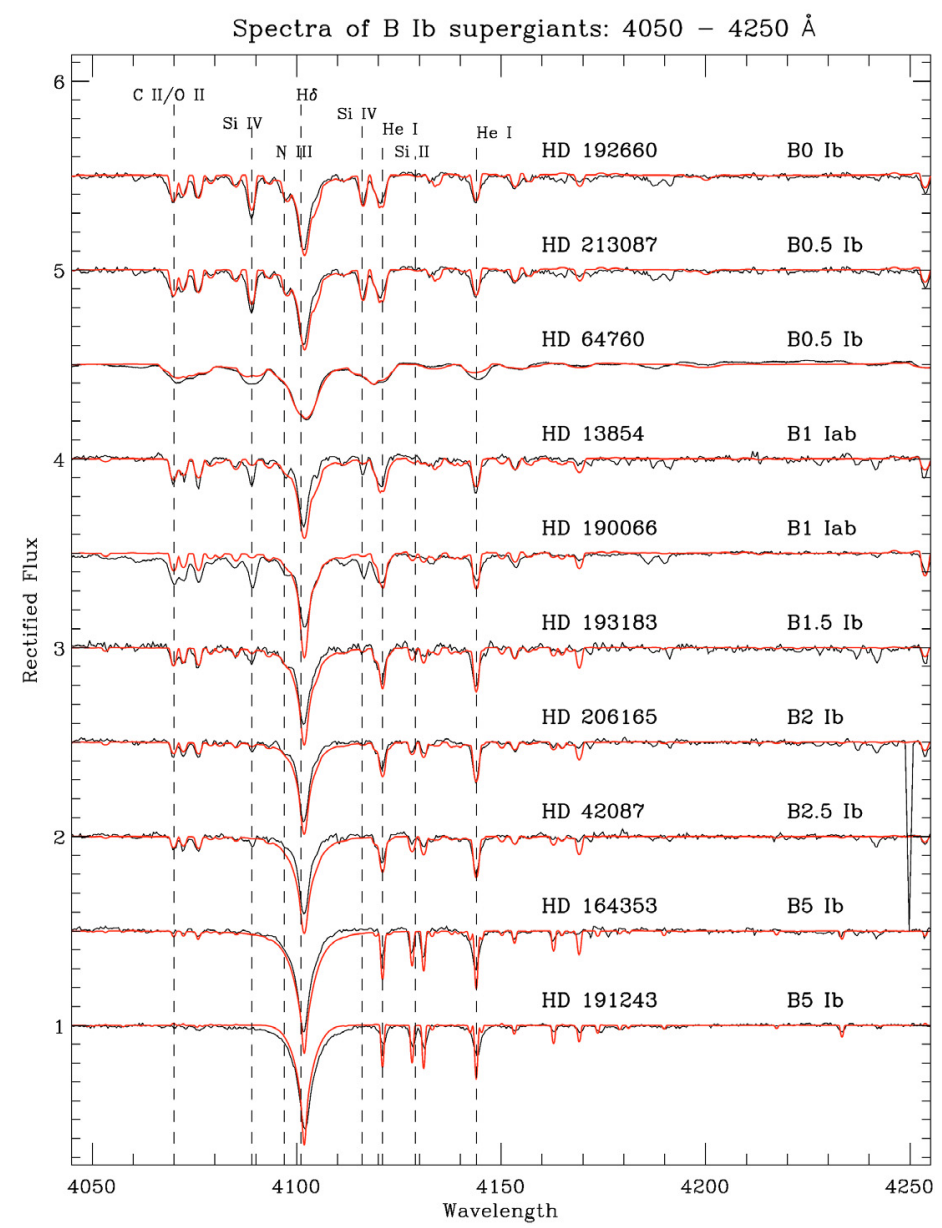

Fig. A.4. CMFGEN model fits (4050-4250 $\AA$ ) to the optical spectra of $10 \mathrm{~B}$ Ib supergiants, with the $T_{\text {eff }}$, luminosity and CNO diagnostic lines marked as shown. Optical spectrum is in black, CMFGEN model fit is shown in red. 
S. C. Searle et al.: Fundamental parameters of Galactic B supergiants. I., Online Material p 6

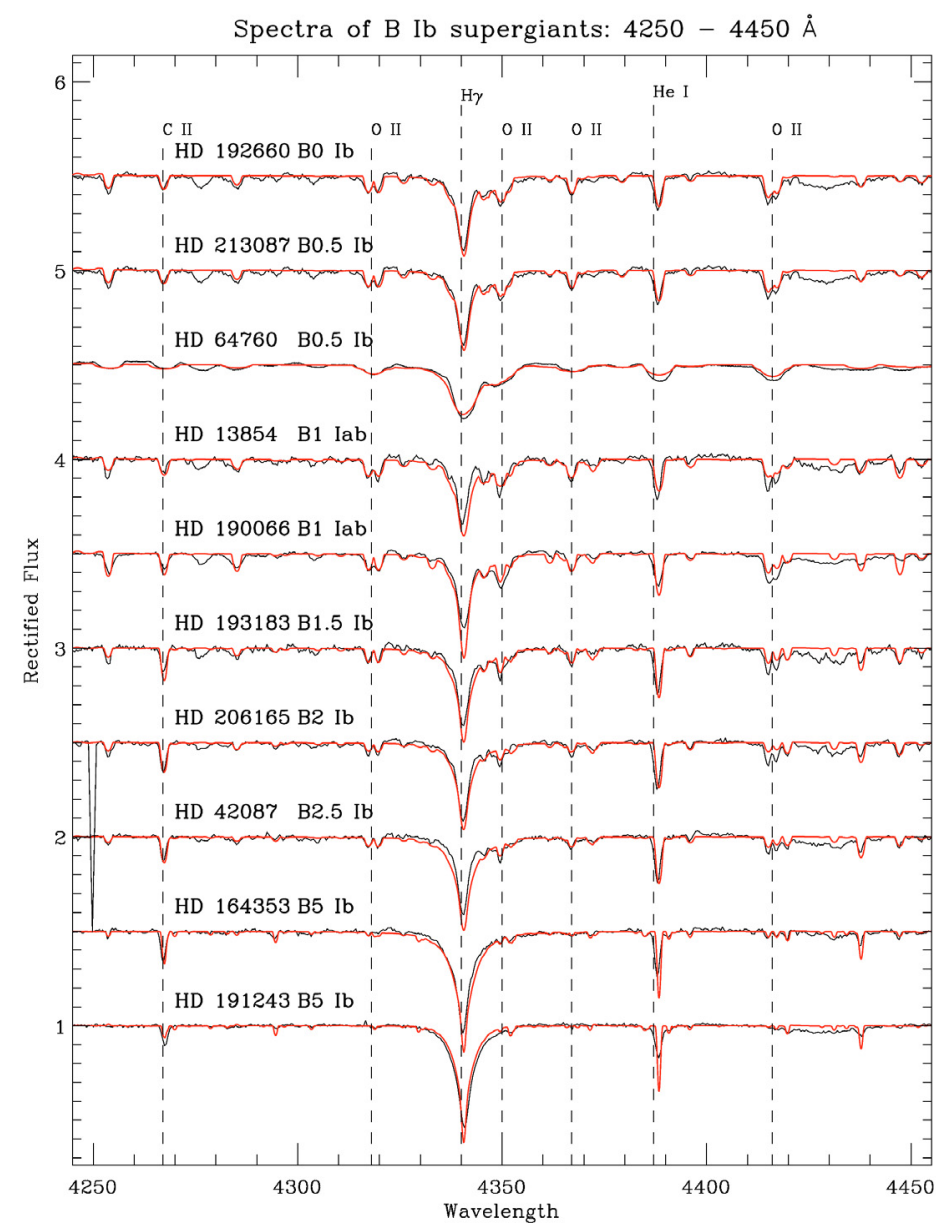

Fig. A.5. CMFGEN model fits (4250-4450 $\AA$ ) to the optical spectra of $10 \mathrm{~B}$ Ib supergiants, with the $T_{\text {eff }}$, luminosity and CNO diagnostic lines marked as shown. Optical spectrum is in black, CMFGEN model fit is shown in red. 
S. C. Searle et al.: Fundamental parameters of Galactic B supergiants. I., Online Material $p 7$

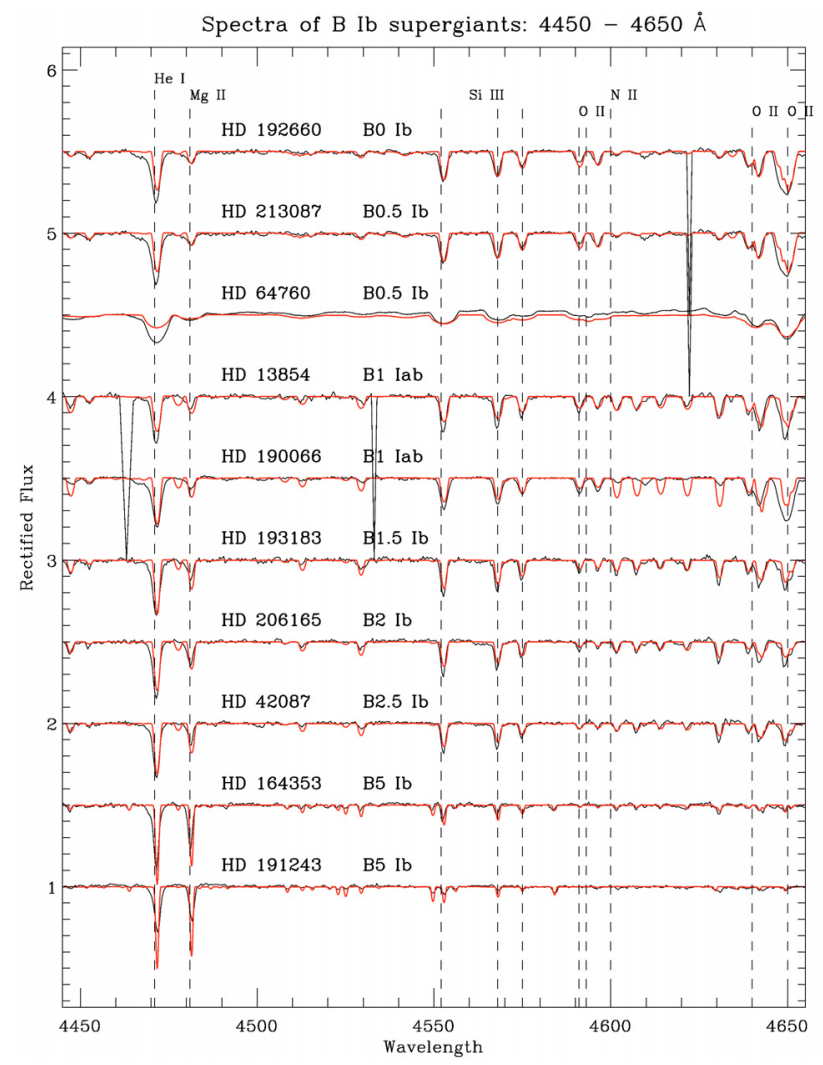

Fig. A.6. CMFGEN model fits (4450-4650 $\AA$ ) to the optical spectra of $10 \mathrm{~B}$ Ib supergiants, with the $T_{\text {eff }}$, luminosity and CNO diagnostic lines marked as shown. Optical spectrum is in black, CMFGEN model fit is shown in red. 
S. C. Searle et al.: Fundamental parameters of Galactic B supergiants. I., Online Material $p 8$
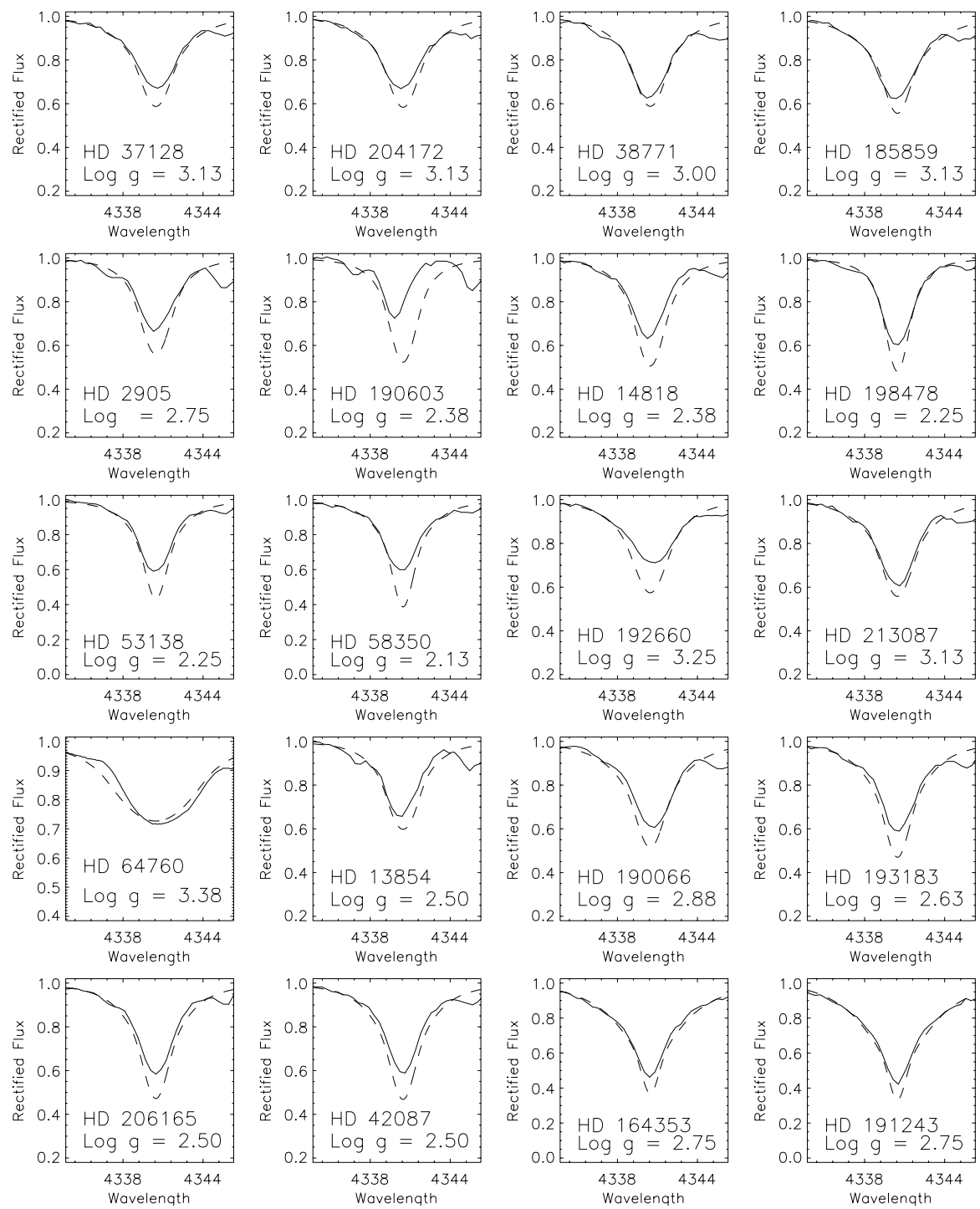

Fig. A.7. TLUSTY model fits to the $\mathrm{H} \gamma$ profile of all 20 supergiants. Optical spectrum is represented by a solid, black line; TLUSTY model fit is shown as a dotted black line. 
S. C. Searle et al.: Fundamental parameters of Galactic B supergiants. I., Online Material p 9
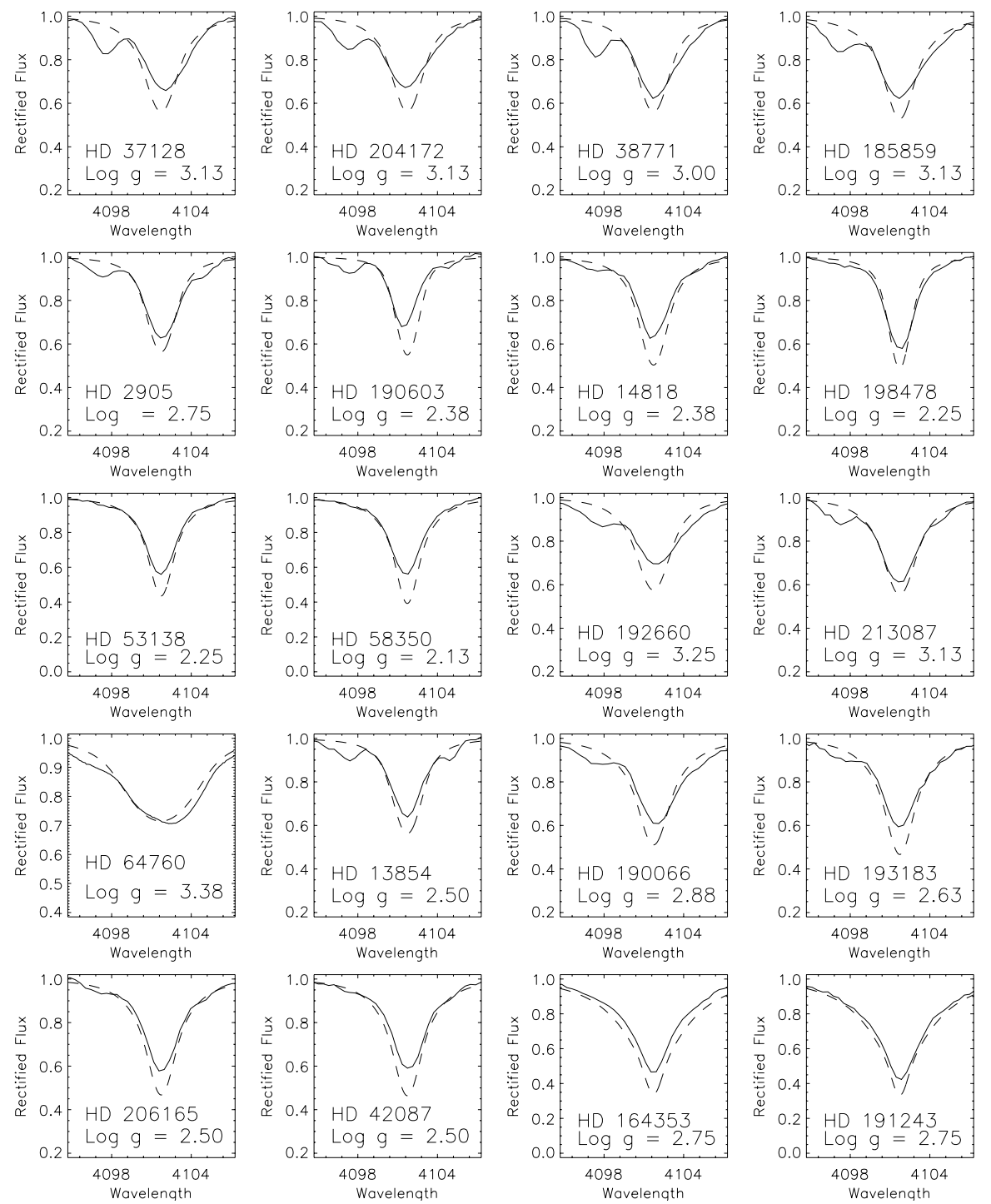

Fig. A.8. TLUSTY model fits to the $\mathrm{H} \delta$ profile of all 20 supergiants. Optical spectrum is represented by a solid, black line; TLUSTY model fit is shown as a dotted black line. 
S. C. Searle et al.: Fundamental parameters of Galactic B supergiants. I., Online Material p 10

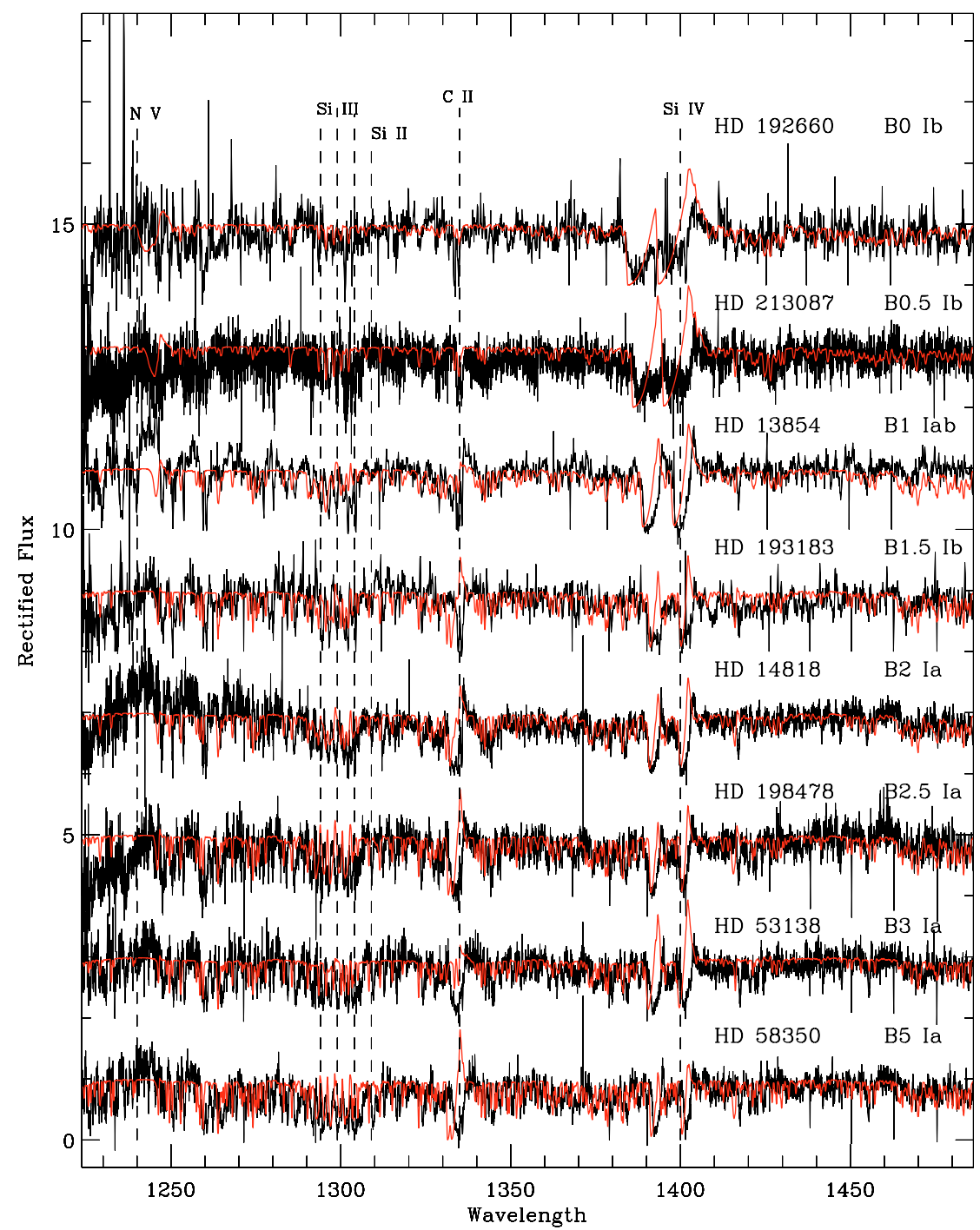

Fig. A.9. CMFGEN model fit to the IUE spectra of 10 B0-B5 supergiants (1230-1480 ̊̊). The solid red line represents the model fits whereas the solid black line is the IUE spectrum. 
S. C. Searle et al.: Fundamental parameters of Galactic B supergiants. I., Online Material p 11

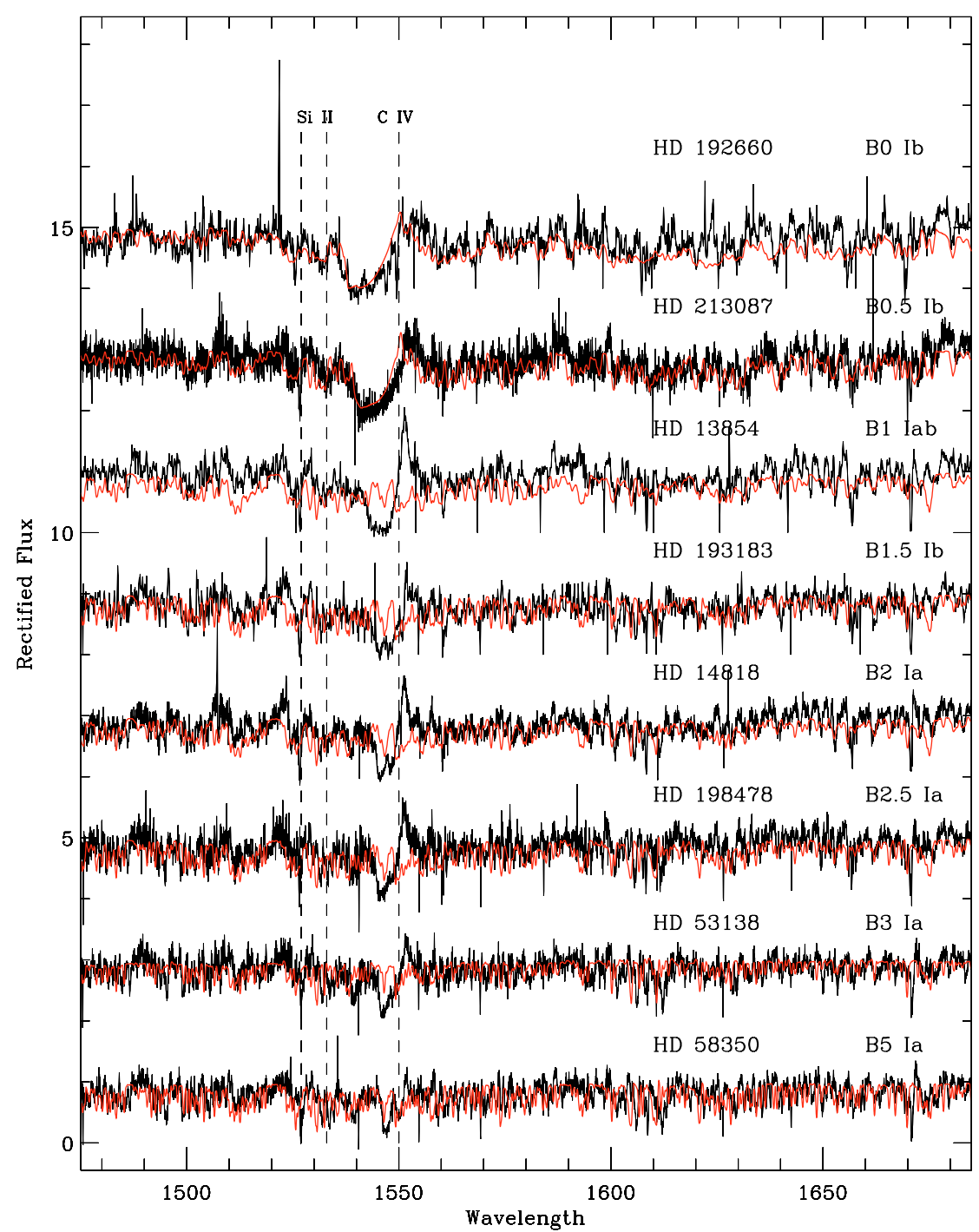

Fig. A.10. CMFGEN model fit to the IUE spectra of 10 B0-B5 supergiants (1480-1680 $\AA$ ). The solid red line represents the model fits whereas the solid black line is the IUE spectrum. 
S. C. Searle et al.: Fundamental parameters of Galactic B supergiants. I., Online Material p 12

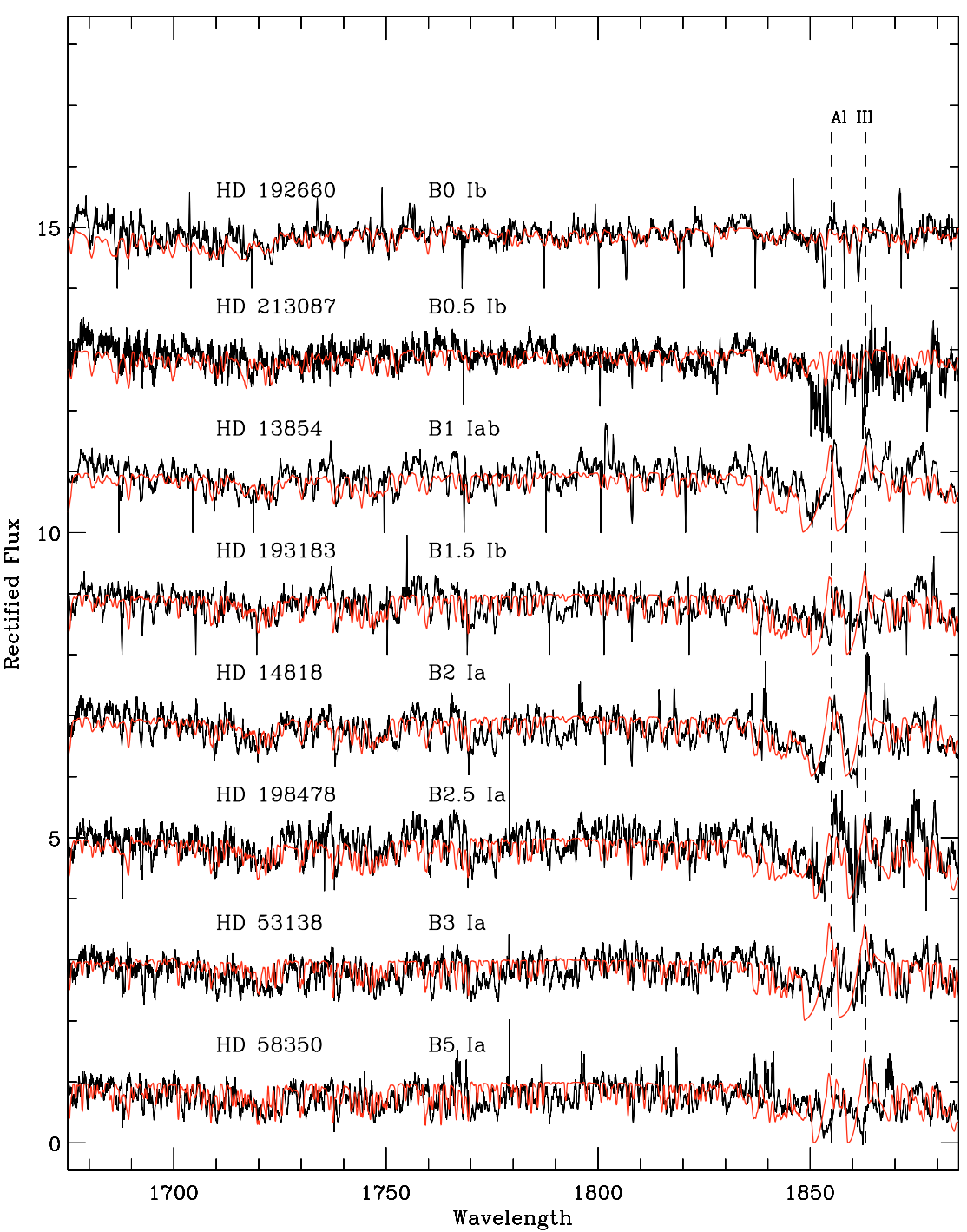

Fig. A.11. CMFGEN model fit to the IUE spectra of 10 B0-B5 supergiants (1680-1880 $\AA$ ). The solid red line represents the model fits whereas the solid black line is the IUE spectrum. 